\title{
CELLULAR DECOMPOSITIONS FOR NESTED HILBERT SCHEMES OF POINTS
}

\author{
JAN CHEAH
}

\begin{abstract}
In this paper, we study the smoothness, tangent spaces, structure and homology groups of various "nested" Hilbert schemes parametrizing nests $Z_{1} \subset Z_{2} \subset \ldots \subset Z_{m}$ of zerodimensional subschemes on smooth quasiprojective varieties.
\end{abstract}

\subsection{Basic notation.}

Suppose that $X$ is a quasiprojective scheme defined over the complex numbers. We denote the Hilbert scheme parametrizing zero-dimensional subschemes of length $n(n \geq 1)$ of $X$ by $\operatorname{Hilb}^{n} X$. A nested Hilbert scheme is defined to be a scheme of the form

$$
\begin{aligned}
& Z_{\mathbf{n}}(X):=\left\{\left(Z_{1}, Z_{2}, \ldots, Z_{m}\right): Z_{i} \in \operatorname{Hilb}^{n_{i}} X\right. \\
&\text { and } \left.Z_{i} \text { is a subscheme of } Z_{j} \text { if } i<j\right\},
\end{aligned}
$$

where the symbol $\mathbf{n}$ is used as a shorthand for the $m$-tuple $\left(n_{1}, n_{2}, . ., n_{m}\right)$. This shorthand will be used throughout the paper. There is, of course, no loss of generality in assuming that $n_{1}<n_{2}<\ldots<n_{m}$ when we study such a space. The construction of the spaces $Z_{\mathbf{n}}(X)$ as schemes is a simple modification of the construction of $\mathrm{Hilb}^{n} X$ found in $[\mathbf{G r}]$, [Kol] and [Mum] (see [Ch1] for details).

We also consider the reduced scheme parametrizing zero-dimensional subschemes of length $n(n \geq 1)$ of a smooth variety $X$ concentrated at some fixed point $P$. If $X$ has dimension $r$, this scheme can be identified with the reduced scheme parametrizing zero-dimensional subschemes of length $n$ of $\mathbb{A}^{r}$ concentrated at the origin and is hence denoted by $\operatorname{Hilb}^{n}\left(\mathbb{A}^{r}, 0\right)$. Alternatively, $\operatorname{Hilb}^{n}\left(\mathbb{A}^{r}, 0\right)$ agrees with the reduced scheme of $\operatorname{Hilb}^{n}\left(\operatorname{Spec}\left(\mathbb{C}\left[\left[x_{1}, x_{2}, \ldots\right.\right.\right.\right.$, $\left.\left.\left.x_{r}\right]\right]\right)$ ), the Hilbert scheme parametrizing subschemes of length $n$ of Spec $\left(\mathbb{C}\left[\left[x_{1}, x_{2}, \ldots, x_{r}\right]\right]\right)$. As before, one can consider nested Hilbert schemes of the form

$$
\begin{aligned}
\mathcal{Z}_{\mathbf{n}}\left(\mathbb{A}^{r}, 0\right):=\left\{\left(Z_{1}, Z_{2}, \ldots, Z_{m}\right): Z_{i}\right. & \in \operatorname{Hilb}^{n_{i}}\left(\mathbb{A}^{r}, 0\right) \\
& \text { and } \left.Z_{i} \text { is a subscheme of } Z_{j} \text { if } i<j\right\}
\end{aligned}
$$

and these nested Hilbert schemes are referred to as the "punctual" nested Hilbert schemes. 
Except where otherwise stated, we restrict our investigations to the nested Hilbert schemes on smooth connected quasiprojective varieties. (By a variety, we mean a seperated reduced scheme of finite type over $\mathbb{C}$.) The assumption of smoothness is a real condition which is necessary for the arguments we employ but connectedness is assumed only for convenience; there is no loss of generality since the Hilbert scheme of points of a variety is the product of the Hilbert schemes of points of its components.

\subsection{Summary of results.}

If $C$ is a smooth curve, Hilb ${ }^{n} C$ is isomorphic to the $n^{\text {th }}$ symmetric product of $C$, which is a smooth variety; in fact, any nested Hilbert scheme $Z_{\mathbf{n}}(C)$ is smooth because it is a product of symmetric products of $C$ (see, for example, [Ch1], Chapter IV, Corollary 2.3). If $S$ is a smooth surface, all the Hilbert schemes Hilb $^{n} S$ are smooth $[\mathbf{F o}]$, as are nested Hilbert schemes of the form $Z_{n-1, n}(S)$, which are of much interest in Donaldson theory [Tik]. It is also well-known that whenever $X$ is smooth, the schemes $\operatorname{Hilb}^{2} X$, Hilb ${ }^{3} X$, $Z_{1,2}(X)$ and $Z_{2,3}(X)$ are smooth [Elen-LeB].

In fact, we prove here that if $X$ is a smooth quasiprojective $r$-dimensional variety, the nested Hilbert scheme $Z_{\mathbf{n}}(X)$ is smooth precisely when either

$$
\left\{\begin{array}{l}
\text { (a) } r \leq 1 \text { or } \\
\text { (b) } r=2 \text { and } Z_{\mathbf{n}}(X) \text { is equal to } \operatorname{Hilb}^{n} X \text { or } Z_{n-1, n}(X) \text { for some } n \text { or } \\
\text { (c) } r \geq 3 \text { and } Z_{\mathbf{n}}(X) \text { is equal to one of the spaces } \\
\operatorname{Hilb}^{1} X, \operatorname{Hilb}^{2} X, \operatorname{Hilb}^{3} X, Z_{1,2}(X) \text { or } Z_{2,3}(X) .
\end{array}\right.
$$

In Chapter I, we show that a singular point can be constructed on any nested Hilbert scheme not included in the list above. Ellingsrud and Strømme's $\mathbb{C}^{*}$-action on Hilb $^{n} \mathbb{P}^{2}[\mathbf{E}-\mathbf{S}]$ can be generalized in an obvious way $[\S 3.1]$ to give a $\mathbb{C}^{*}$-action with isolated fixed points on any nested Hilbert scheme $Z_{\mathbf{n}}\left(\mathbb{P}^{r}\right)$ and we check in $\S 3.2$ that all of the fixed points on the nested Hilbert schemes which satisfy (0.2.1) are smooth points.

By studying this $\mathbb{C}^{*}$-action on Hilb $\mathbb{P}^{2}$ and applying a theorem of Bialynicki-Birula, Ellingsrud and Strømme obtain cellular decompositions of the spaces $\operatorname{Hilb}^{n} \mathbb{P}^{2}, \operatorname{Hilb}^{n} \mathbb{A}^{2}$ and $\operatorname{Hilb}^{n}\left(\mathbb{A}^{2}, 0\right)$, enabling them to compute the Borel-Moore homology groups of these spaces and to deduce that they coincide with the Chow groups; we may likewise obtain [§3.3] cellular decompositions and study the homology groups of all the smooth nested Hilbert schemes on affine and projective space as well as of the corresponding punc- 
tual nested Hilbert schemes

$$
\begin{aligned}
& \mathcal{Z}_{\mathbf{n}}\left(\mathbb{A}^{1}, 0\right), \operatorname{Hilb}^{n}\left(\mathbb{A}^{2}, 0\right), \mathcal{Z}_{n-1, n}\left(\mathbb{A}^{2}, 0\right), \\
& \operatorname{Hilb}^{2}\left(\mathbb{A}^{r}, 0\right)\left(=\mathcal{Z}_{1,2}\left(\mathbb{A}^{r}, 0\right)\right), \operatorname{Hilb}^{3}\left(\mathbb{A}^{r}, 0\right) \text { and } \mathcal{Z}_{2,3}\left(\mathbb{A}^{r}, 0\right) .
\end{aligned}
$$

Whereas Ellingsrud and Strømme count the dimension of the "positive part" of the tangent space at each of the $\mathbb{C}^{*}$-fixed points of $\mathrm{Hilb}^{n} \mathbb{P}^{2}$ by the use of some exact sequences, we actually write down [§2.5 and $\S 2.6]$ an explicit weight basis for the tangent space at each of the $\mathbb{C}^{*}$-fixed points of $\mathrm{Hilb}^{n} \mathbb{P}^{2}$ and $Z_{n-1, n}\left(\mathbb{P}^{2}\right)$.

Moreover, just as the $\mathbb{C}^{*}$-action on $\operatorname{Hilb}^{n}\left(\mathbb{P}^{2}\right)$ can be chosen [Gö1, Gö2] so that the resulting cellular decomposition of $\operatorname{Hilb}^{n}\left(\mathbb{A}^{2}, 0\right)$ is a union of cellular decompositions of its Hilbert function strata, one can obtain [§3.4] cellular decompositions of the "Hilbert function strata" of the punctual nested Hilbert schemes listed in (0.2.2). Indeed, the geometry of these spaces closely resembles that of the Hilbert function strata of $\operatorname{Hilb}^{n}\left(\mathbb{A}^{2}, 0\right)$. For example, each Hilbert function stratum of $\mathcal{Z}_{n-1, n}\left(\mathbb{A}^{2}, 0\right)$ is [§3.4] an irreducible smooth variety and its intersection with the closed subset of $\mathcal{Z}_{n-1, n}\left(\mathbb{A}^{2}, 0\right)$ parametrizing pairs of subschemes defined by homogeneous ideals is an irreducible smooth projective variety.

With an appropriate interpretation of the word "homology", all our results apart from some in Section 3.4 remain valid over any algebraically closed base field $k$. In the last part of Section 3.4.b (the part following Figure 10), we list some results from [Iar] about the Hilbert function strata of $\operatorname{Hilb}^{n}\left(\mathbb{A}^{2}, 0\right)$ which are proved for algebraically closed fields $k$ of characteristic zero (or for which char $k \geq n$ ). These results are then used in Section 3.4.c to study the Hilbert function strata of the punctual nested Hilbert schemes $\mathcal{Z}_{n-1, n}\left(\mathbb{A}^{2}, 0\right)$. While it is still true that each Hilbert function stratum of $\mathcal{Z}_{n-1, n}\left(\mathbb{A}^{2}, 0\right)$ has the cell decomposition described in Proposition 3.4.15.2 when the characteristic of $k$ is finite, it does not follow from our argument that it is a smooth space. We also do not know whether the associated space of homogeneous ideals is smooth or if it has a cellular decomposition.

The cellular decompositions obtained here for the punctual nested Hilbert schemes are used in the sequels to this paper [Ch2] and [Ch3] to calculate the virtual Hodge polynomials of all the smooth nested Hilbert schemes on $X$ (and of related varieties such as the universal families over some of these nested Hilbert schemes) in terms of the virtual Hodge polynomial of $X$ for any smooth quasiprojective variety $X$.

This paper is part of my thesis [Ch1] written under the guidance of Bill Fulton. Thanks go also to the referee for his many helpful remarks. 


\subsection{Description of contents.}

In Chapter I, we prove that if $X$ is a smooth $r$-dimensional quasiprojective variety, the nested Hilbert scheme $Z_{\mathbf{n}}(X)$ is singular unless it satisfies (0.2.1).

In Chapter II, we study certain $\mathbb{C}^{*}$-actions on the nested Hilbert schemes on $\mathbb{A}^{r}$ which satisfy (0.2.1). In particular, we construct explicit weight basis for the tangent spaces to these schemes at the $\mathbb{C}^{*}$-fixed points. The results obtained in this chapter are needed in Chapter III. The cases Hilb ${ }^{n} \mathbb{A}^{2}$ and $Z_{n-1, n}\left(\mathbb{A}^{2}\right)$ are the most involved and take up the bulk of the chapter.

In Chapter III, the nested Hilbert schemes which satisfy (0.2.1) are shown to be smooth. We look at $\mathbb{C}^{*}$-actions on all the smooth nested Hilbert schemes on $\mathbb{P}^{r}$ to obtain cellular decompositions for these spaces, for the smooth nested Hilbert schemes on $\mathbb{A}^{r}$ and for the corresponding punctual nested Hilbert schemes.

A consequence of the existence of cellular decompositions for these spaces is that the cycle maps between their Chow groups and their Borel-Moore homology groups are isomorphisms. In particular, there is no odd homology and the homology groups are all free. The number of cells of each dimension or, equivalently, the Betti numbers of the punctual nested Hilbert schemes listed in (0.2.2) are computed in Section 3.3; the number of cells of each dimension of the smooth nested Hilbert schemes on affine and projective space can be read off from formulae which we obtain in [Ch2] and [Ch3].

Lastly, in Section 3.4, we study the "Hilbert function strata" of the punctual nested Hilbert schemes listed in (0.2.2) and obtain cellular decompositions for these spaces.

\subsection{Essential facts about Hilbert schemes.}

Connectedness. In $[\mathbf{F o}]$, Fogarty shows that $\operatorname{Hilb}^{n}\left(\mathbb{A}^{r}, 0\right)$ is connected and deduces that $\operatorname{Hilb}^{n} X$ is connected when $X$ is a connected quasiprojective scheme. It is reasonably straightforward to adapt his proof to show that all the spaces $\mathcal{Z}_{\mathbf{n}}\left(\mathbb{A}^{r}, 0\right)$ and $Z_{\mathbf{n}}(X)$ are likewise connected (details can be found in $[\mathrm{Ch} 1])$.

Tangent spaces. If $X=\operatorname{Spec} R$, there is a canonical isomorphism between $\operatorname{Hom}_{R}(I, R / I)$ and the tangent space to the Hilbert scheme Hilb ${ }^{n}$ at the point representing the subscheme of $X$ associated to the ideal $I[\mathbf{G r}]$.

One checks easily that the tangent space to $Z_{n_{1}, n_{2}}(X)$ at the point corresponding to the pair of ideals $\left(I_{1}, I_{2}\right)$ is canonically isomorphic to $\operatorname{Ker}(\phi-\psi)$, where

$$
\begin{aligned}
& \phi: \operatorname{Hom}_{R}\left(I_{1}, R / I_{1}\right) \rightarrow \operatorname{Hom}_{R}\left(I_{2}, R / I_{1}\right) \text { and } \\
& \psi: \operatorname{Hom}_{R}\left(I_{2}, R / I_{2}\right) \rightarrow \operatorname{Hom}_{R}\left(I_{2}, R / I_{1}\right)
\end{aligned}
$$


are the obvious maps and

$$
(\phi-\psi): \operatorname{Hom}_{R}\left(I_{1}, R / I_{1}\right) \oplus \operatorname{Hom}_{R}\left(I_{2}, R / I_{2}\right) \rightarrow \operatorname{Hom}_{R}\left(I_{2}, R / I_{1}\right)
$$

is defined by letting $(\phi-\psi)\left(f_{1}, f_{2}\right)=\phi\left(f_{1}\right)-\psi\left(f_{2}\right)$.

Similarly, the tangent space to $Z_{\mathbf{n}}(X)$ at the point corresponding to the $m$-tuple of ideals $\left(I_{1}, I_{2}, \ldots, I_{m}\right)$ is canonically isomorphic to $\underset{i<j}{\cap} \operatorname{Ker}\left(\left(\phi_{i, j}-\right.\right.$ $\left.\left.\psi_{i, j}\right) \circ \pi_{i, j}\right)$, where

$$
\pi_{i, j}: \underset{k=1}{m} \operatorname{Hom}_{R}\left(I_{k}, R / I_{k}\right) \rightarrow \operatorname{Hom}_{R}\left(I_{i}, R / I_{i}\right) \oplus \operatorname{Hom}_{R}\left(I_{j}, R / I_{j}\right)
$$

is the projection map, and where

$$
\begin{aligned}
& \phi_{i, j}: \operatorname{Hom}_{R}\left(I_{i}, R / I_{i}\right) \rightarrow \operatorname{Hom}_{R}\left(I_{j}, R / I_{i}\right) \quad \text { and } \\
& \psi_{i, j}: \operatorname{Hom}_{R}\left(I_{j}, R / I_{j}\right) \rightarrow \operatorname{Hom}_{R}\left(I_{j}, R / I_{i}\right)
\end{aligned}
$$

are the obvious morphisms.

\section{Chapter I. Towards the classification of the smooth nested Hilbert schemes of points.}

In this chapter, we prove the following:

Theorem. If $X$ is a smooth $r$-dimensional quasiprojective variety, the nested Hilbert scheme $Z_{\mathbf{n}}(X)$ is singular unless it satisfies (0.2.1).

The converse is proved in Section 3.2.

We need only consider the case when $X$ is $\mathbb{A}^{r}$ since any point of $Z_{\mathbf{n}}\left(\mathbb{A}^{r}\right)$ has a neighbourhood in the classical topology which is analytically isomorphic to a neighbourhood of some point of $Z_{\mathbf{n}}(X)$. The theorem is, therefore, a consequence of the four following lemmas.

Lemma 1.1. If $r \geq 2$ and $m \geq 3$, the nested Hilbert scheme $Z_{\mathbf{n}}\left(\mathbb{A}^{r}\right)$ is singular.

Lemma 1.2. If $r \geq 2$ and $n_{2}-n_{1}>1$, the nested Hilbert scheme $Z_{n_{1}, n_{2}}\left(\mathbb{A}^{r}\right)$ is singular.

Lemma 1.3. If $r \geq 3$ and $n \geq 4$, the nested Hilbert scheme $Z_{n-1, n}\left(\mathbb{A}^{r}\right)$ is singular.

Lemma 1.4. If $r \geq 3$ and $n \geq 4$, the Hilbert scheme $\operatorname{Hilb}^{n}\left(\mathbb{A}^{r}\right)$ is singular.

Let $\operatorname{Hilb}_{\neq}^{n} \mathbb{A}^{r}$ denote the open subset of Hilb ${ }^{n} \mathbb{A}^{r}$ consisting of $n$ distinct points and let $p_{m}$ denote the projection from $Z_{\mathbf{n}}\left(\mathbb{A}^{r}\right)$ to $\operatorname{Hilb}^{n_{m}} \mathbb{A}^{r}$. Then, the 
nested Hilbert scheme $Z_{\mathbf{n}}\left(\mathbb{A}^{r}\right)$ is a connected scheme with an open subset $p_{m}^{-1}\left(\operatorname{Hilb}_{\neq}^{n} \mathbb{A}^{r}\right)$ of dimension $r n_{m}$. Thus, to show that a given nested Hilbert scheme $Z_{\mathbf{n}}\left(\mathbb{A}^{r}\right)$ is singular, we need only show that it has a point at which the dimension of the Zariski tangent space is larger than $r n_{m}$.

\subsection{Proof of Lemma 1.1.}

First of all, we show that $Z_{1,2,3}\left(\mathbb{A}^{r}\right)(r \geq 2)$ is singular by constructing a point $Q_{1}=\left(Z_{1}, Z_{2}, Z_{3}\right)$ on $Z_{1,2,3}\left(\mathbb{A}^{r}\right)$ at which the tangent space has dimension greater than $3 r$. Let $Z_{1}, Z_{2}$ and $Z_{3}$ be the subschemes of $\mathbb{A}^{r}$ associated to the ideals of $\mathbb{C}\left[x_{1}, x_{2}, \ldots, x_{r}\right], I_{1}=\left\langle x_{1}, x_{2}, \ldots, x_{r}\right\rangle, I_{2}=\left\langle x_{1}^{2}, x_{2}, x_{3}, \ldots, x_{r}\right\rangle$ and $I_{3}=\left\langle x_{1}^{2}, x_{1} x_{2}, x_{2}^{2}, x_{3}, \ldots, x_{r}\right\rangle$.

We call the generators of $I_{i}$ given above its canonical generators. If $\alpha$ is a canonical generator of $I_{i}$ and $\beta$ is an element of $R / I_{i}$, there exists at most one $R$-module homomorphism from $I_{i}$ to $R / I_{i}$ which takes $\alpha$ to $\beta$ and all the other canonical generators of $I_{i}$ to zero; when such a homomorphism exists, we denote it by $(\alpha \mapsto \beta)$.

The $(3 r+1)$ elements of $\operatorname{Hom}_{R}\left(I_{1}, R / I_{1}\right) \oplus \operatorname{Hom}_{R}\left(I_{2}, R / I_{2}\right) \oplus \operatorname{Hom}_{R}\left(I_{3}\right.$, $\left.R / I_{3}\right)$,

$$
\begin{aligned}
& \left(x_{k} \mapsto 1, \quad x_{k} \mapsto 1, \quad x_{k} \mapsto 1 \quad\right) \text { for } 3 \leq k \leq r,\left(x_{2} \mapsto 1, x_{2} \mapsto 1, x_{1} x_{2} \mapsto x_{1}\right),
\end{aligned}
$$

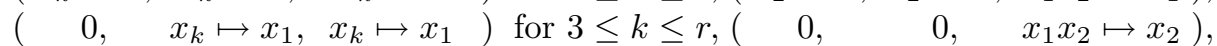

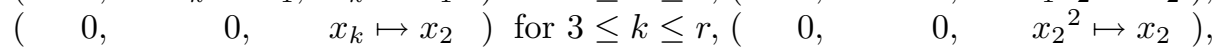

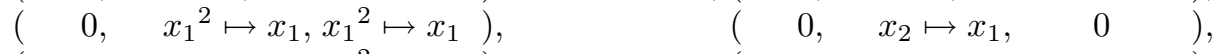

$$
\begin{aligned}
& \left(\begin{array}{llllll}
0, & 0, & x_{1}^{2} \mapsto x_{2}
\end{array}\right), \quad\left(\begin{array}{llll}
x_{1} \mapsto 1, & 0, & 0
\end{array}\right) \text {, }
\end{aligned}
$$

are, by direct calculation, linearly independent elements of the tangent space to $Z_{1,2,3}\left(\mathbb{A}^{r}\right)$ at $Q_{1}=\left(Z_{1}, Z_{2}, Z_{3}\right)$.

Next, we show that given any nested Hilbert scheme $Z_{\mathbf{n}}\left(\mathbb{A}^{r}\right)$ with $r \geq 2$ and $m \geq 3$, we may construct a point $Q_{2}$ on it at which the tangent space has dimension greater than $r n_{m}$. Let $P_{1}, P_{2}, \ldots, P_{n_{m}-3}$ be distinct points of $\mathbb{A}^{r} \backslash\{0\}$. For $1 \leq i \leq 3$, let $k_{i}=n_{i}-i$ and let $Z_{i}^{\prime}=Z_{i} \cup\left(\bigcup_{j=1}^{k_{i}} P_{i}\right)$ while for $3 \leq i \leq m$, let $k_{i}=n_{i}-3$ and let $Z_{i}^{\prime}=Z_{3} \cup\left(\bigcup_{j=1}^{k_{i}} P_{i}\right)$. We check that $Q_{2}=\left(Z_{1}, Z_{2}, \ldots, Z_{m}\right)$ is a point of $Z_{\mathbf{n}}\left(\mathbb{A}^{r}\right)$. Denoting the tangent space to $X$ at the point $P$ by $T_{P, X}$, we have $T_{Q_{2}, Z_{\mathbf{n}}\left(\mathbb{A}^{r}\right)} \simeq T_{Q_{1}, Z_{1,2,3}\left(\mathbb{A}^{r}\right)} \oplus\left(\underset{j=1}{\bigoplus} T_{P_{j}, \mathbb{A}^{r}}\right)$, so the dimension of $T_{Q_{2}, Z_{\mathbf{n}}\left(\mathbb{A}^{r}\right)}$ is greater than $r n_{m}$ as required.

\subsection{Proof of Lemma 1.2.}

We retain the notation of the previous section and start by showing that when $r \geq 2$, the tangent space to $Z_{1,3}\left(\mathbb{A}^{r}\right)$ at the point $Q_{3}=\left(Z_{1}, Z_{3}\right)$ has 
dimension greater than $3 r$. We do so by checking that the $3 r+2$ elements of $\operatorname{Hom}_{R}\left(I_{1}, R / I_{1}\right) \oplus \operatorname{Hom}_{R}\left(I_{3}, R / I_{3}\right)$,

$$
\begin{aligned}
& \left(\quad 0, \quad x_{k} \mapsto x_{1} \quad\right) \text { for } 3 \leq k \leq r,\left(\quad 0, \quad x_{1} x_{2} \mapsto x_{2}\right) \text {, } \\
& \left(\begin{array}{ll}
0, & x_{k} \mapsto x_{2}
\end{array}\right) \text { for } 3 \leq k \leq r,\left(\quad 0, \quad x_{2}{ }^{2} \mapsto x_{1}\right) \text {, } \\
& \left(x_{k} \mapsto 1, \quad x_{k} \mapsto 1 \quad\right) \text { for } 3 \leq k \leq r,\left(\quad 0, \quad x_{2}{ }^{2} \mapsto x_{2}\right),
\end{aligned}
$$

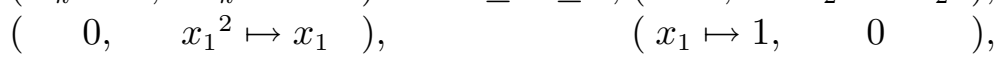

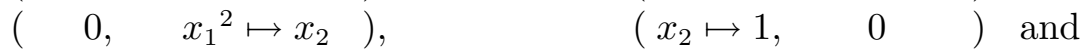

$$
\begin{aligned}
& \left(\quad 0, \quad x_{1} x_{2} \mapsto x_{1}\right) \text {, }
\end{aligned}
$$

are, in fact, linearly independent elements of the tangent space to $Z_{1,3}\left(\mathbb{A}^{r}\right)$ at $Q_{3}=\left(Z_{1}, Z_{3}\right)$.

Then, we show that given any nested Hilbert scheme $Z_{n_{1}, n_{2}}\left(\mathbb{A}^{r}\right)$ with $r \geq 2$ and $n_{2}-n_{1}>1$, we may construct a point $Q_{4}$ on it at which the tangent space has dimension greater than $r n_{2}$. Let $P_{1}, P_{2}, \ldots, P_{n_{2}-3}$ be distinct points on $\mathbb{A}^{r} \backslash\{0\}$, let $\tilde{Z}_{1}=Z_{1} \cup \bigcup_{j=1}^{n_{1}-1} P_{j}$ and let $\tilde{Z}_{2}=Z_{3} \cup \bigcup_{j=1}^{n_{2}-3} P_{j}$. Then, $Q_{4}=\left(\tilde{Z}_{1}, \tilde{Z}_{2}\right)$ is a point of $Z_{n_{1}, n_{2}}\left(\mathbb{A}^{r}\right)$ and $T_{Q_{4}, Z_{n_{1}, n_{2}}\left(\mathbb{A}^{r}\right)}$ is isomorphic to $T_{Q_{3}, Z_{1,3}\left(\mathbb{A}^{r}\right)} \oplus\left(\bigoplus_{j=1}^{n_{2}-3} T_{P_{j}, \mathbb{A}^{r}}\right)$, so the dimension of $T_{Q_{4}, Z_{n_{1}, n_{2}}\left(\mathbb{A}^{r}\right)}$ is greater than $r n_{2}$ as required.

\subsection{Proof of Lemma 1.3.}

First, we show that $Z_{3,4}\left(\mathbb{A}^{r}\right)(r \geq 3)$ is singular by constructing a point $\left(Y_{1}, Y_{2}\right)$ on $Z_{3,4}\left(\mathbb{A}^{r}\right)$ at which the tangent space has dimension greater than $4 r$. Let $Y_{1}$ and $Y_{2}$ be the subschemes of $\mathbb{A}^{r}$ associated to the ideals $J_{1}=$ $\left\langle x_{1}^{2}, x_{2}{ }^{2}, x_{1} x_{2}, x_{3}, \ldots, x_{r}\right\rangle$ and $J_{2}=\left\langle x_{1}{ }^{2}, x_{2}{ }^{2}, x_{3}{ }^{2}, x_{1} x_{2}, x_{1} x_{3}, x_{2} x_{3}, x_{4}, x_{5}\right.$, $\left.\ldots, x_{r}\right\rangle$ of $\mathbb{C}\left[x_{1}, x_{2}, \ldots, x_{r}\right]$. We call the generators of $J_{i}$ given above its canonical generators. Then, with the notation of Section 1.1, the elements of $\operatorname{Hom}_{R}\left(J_{1}, R / J_{1}\right) \oplus \operatorname{Hom}_{R}\left(J_{2}, R / J_{2}\right)$,

$$
\begin{aligned}
& \left(\quad x_{i} \mapsto 1, \quad x_{i} \mapsto 1 \quad\right) \text { for } 4 \leq i \leq r,\left(x_{2}^{2} \mapsto x_{1}, x_{2}{ }^{2} \mapsto x_{1}\right) \text {, } \\
& \left(x_{i} \mapsto x_{1}, \quad x_{i} \mapsto x_{1}\right) \text { for } 4 \leq i \leq r,\left(\quad 0, \quad x_{1} x_{3} \mapsto x_{3}\right),
\end{aligned}
$$

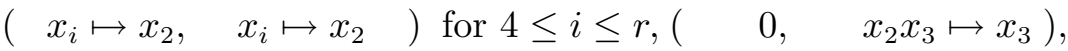

$$
\begin{aligned}
& \left(\begin{array}{ll}
0, & x_{i} \mapsto x_{3}
\end{array}\right) \text { for } 4 \leq i \leq r,\left(\quad 0, \quad x_{3}{ }^{2} \mapsto x_{3}\right) \text {, } \\
& \left(x_{1} x_{2} \mapsto x_{1}, x_{1} x_{2} \mapsto x_{1}\right), \quad\left(x_{3} \mapsto x_{1}, \quad 0 \quad\right), \\
& \left(x_{1} x_{2} \mapsto x_{2}, x_{1} x_{2} \mapsto x_{2}\right), \quad\left(x_{3} \mapsto x_{2}, \quad 0 \quad\right),
\end{aligned}
$$

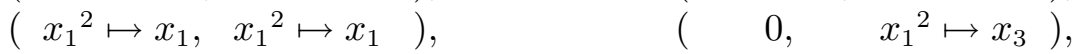

$$
\begin{aligned}
& \left(x_{2}{ }^{2} \mapsto x_{2}, \quad x_{2}{ }^{2} \mapsto x_{2}\right), \quad\left(\quad 0, \quad x_{2}{ }^{2} \mapsto x_{3}\right) \text { and } \\
& \left(x_{1}^{2} \mapsto x_{2}, \quad x_{1}^{2} \mapsto x_{2}\right) \text {, }
\end{aligned}
$$

are $4 r+1$ linearly independent elements of the tangent space to $Z_{3,4}\left(\mathbb{A}^{r}\right)$ at $\left(Y_{1}, Y_{2}\right)$. 
If $P_{1}, P_{2}, \ldots, P_{n-4}$ are $n-4$ distinct points on $\mathbb{A}^{r} \backslash\{0\}$, then $\left(\left(Y_{1} \cup\right.\right.$ $\left.\left.\bigcup_{j=1}^{n-4} P_{i}\right),\left(Y_{2} \cup \cup_{j=1}^{n-4} P_{i}\right)\right)$ is a point of $Z_{n-1, n}\left(\mathbb{A}^{r}\right)$ at which the tangent space has dimension greater than $n r$.

\subsection{Proof of Lemma 1.4.}

We start by showing that for $r \geq 3$, the tangent space to $\mathrm{Hilb}^{4} \mathbb{A}^{r}$ at $Y_{2}$ has dimension greater than $4 r$. In fact, with the notation of the previous sections, the homomorphisms

$$
\begin{aligned}
& \left(x_{i} \mapsto 1\right) \quad \text { for } 4 \leq i \leq r, \quad\left(x_{i} \mapsto x_{3}\right) \quad \text { for } 4 \leq i \leq r, \\
& \left(x_{i} \mapsto x_{1}\right) \quad \text { for } 4 \leq i \leq r, \quad\left(x_{i}{ }^{2} \mapsto x_{j}\right) \quad \text { for } 1 \leq i, j \leq 3 \text {, } \\
& \left(x_{i} \mapsto x_{2}\right) \quad \text { for } 4 \leq i \leq r, \quad\left(x_{i} x_{j} \mapsto x_{i}\right) \quad \text { for } 1 \leq i \neq j \leq 3 \text {, }
\end{aligned}
$$

are $4 r+3$ independent elements of $\operatorname{Hom}_{R}\left(J_{2}, R / J_{2}\right)$.

It remains to show that whenever $r \geq 3$ and $n \geq 4$, the Hilbert scheme Hilb $^{n} \mathbb{A}^{r}$ has a point at which the tangent space has dimension greater than $n r$. If $P_{1}, P_{2}, \ldots, P_{n-4}$ are distinct points of $\mathbb{A}^{r} \backslash\{0\}$, then $\left(Y_{2} \cup \cup_{j=1}^{n-4} P_{j}\right)$ is such a point of $\operatorname{Hilb}^{n} \mathbb{A}^{r}$.

\section{Chapter II. The tangent spaces at the fixed points of various nested Hilbert schemes on affine space under certain actions of the one-dimensional torus.}

The action of $\mathbb{C}^{*}$ on $R=\mathbb{C}\left[x_{1}, x_{2}, \ldots, x_{r}\right]$ given by

$$
t . x=t^{\mu_{i}} x_{i} \text { for } 1 \leq i \leq r
$$

induces an action of $\mathbb{C}^{*}$ on $\operatorname{Hilb}^{n} \mathbb{A}^{r}$, and hence on $Z_{\mathbf{n}}\left(\mathbb{A}^{r}\right)$, as $\mathbb{C}^{*}$ permutes the ideals of $R$ of colength $n$. We restrict our attention to $\mathbb{C}^{*}$-actions with $\left(\mu_{1}, \mu_{2}, \ldots, \mu_{r}\right) \in \mathbb{Z}^{r}$ lying outside the hyperplanes $m_{1} \mu_{1}+m_{2} \mu_{2}+\ldots+m_{r} \mu_{r}=$ 0 for which $\left(m_{1}, m_{2}, \ldots, m_{r}\right)$ is an $r$-tuple of integers between $(-2 n-1)$ and $(2 n+1)$ and for which not all the $m_{i}$ s are zero.

A fixed point of Hilb $\mathbb{A}^{r}$ is a $\mathbb{C}^{*}$-invariant ideal $I$ of $R$ of colength $n$ while a fixed point of $Z_{n-1, n}\left(\mathbb{A}^{r}\right)$ is a pair of invariant ideals $\left(I_{1}, I_{2}\right)$ such that $I_{1}$ contains $I_{2}$, the colength of $I_{1}$ is $n-1$ and the colength of $I_{2}$ is $n$. An invariant ideal $I$ of colength $n$ is generated by monomials in the $x_{j}$ s; there are exactly $n$ monomials in the $x_{j}$ s which are not in $I$ and they form a basis of $R / I$ over $\mathbb{C}$.

Most of this chapter is concerned with investigating the action of $\mathbb{C}^{*}$ on the tangent space $T$ at a fixed point $I$ of $\operatorname{Hilb}^{n} \mathbb{A}^{r}$ and on the tangent space $T^{\prime}$ at a 
fixed point $\left(I_{1}, I_{2}\right)$ of $Z_{n-1, n}\left(\mathbb{A}^{r}\right)$. Again, we use the canonical representations $T=\operatorname{Hom}_{R}(I, R / I)$ and $T^{\prime}=\operatorname{Ker}(\phi-\psi)$.

If $\mathbb{C}^{*}$ acts on a vector space $V$, we denote by $V^{+}$the part of $V$ where the weights of $\mathbb{C}^{*}$ are positive. Here, we calculate

(1) the dimensions of the tangent spaces at the $\mathbb{C}^{*}$-fixed points of various nested Hilbert schemes, and

(2) the dimensions of their positive parts when the action of $\mathbb{C}^{*}$ on $R$ defined in (2.0.1) satisfies $0<\mu_{1}<\mu_{2}<\cdots<\mu_{r}$ and $\mu_{j} / \mu_{i} \gg 0$ whenever $j>i$.

In Chapter III, (1) is used to demonstrate the smoothness of these spaces while (2) is used to obtain cellular decompositions for the associated punctual nested Hilbert schemes.

\subsection{The tangent spaces at the fixed points of $\mathrm{Hilb}^{2} \mathbb{A}^{r}$.}

Let $I_{i}$ be the ideal of $R$ generated by $x_{i}{ }^{2}$ and the $x_{k}$ s for which $1 \leq k \leq r$ and $k \neq i$. There are exactly $r$ fixed points on $\mathrm{Hilb}^{2} \mathbb{A}^{r}$ corresponding to the ideals $I_{1}, I_{2}, \ldots, I_{r}$, these being the only ideals of colength 2 generated by monomials. We will call the $r$ generators of $I_{i}$ described above its canonical generators.

We wish to describe $2 r$ elements $f_{1}, f_{2}, \ldots, f_{r}, g_{1}, g_{2}, \ldots, g_{r}$ of $\operatorname{Hom}_{R}\left(I_{i}\right.$, $R / I_{i}$ ) which take all but one of the canonical generators of $I_{i}$ to zero. If $k \neq i$, let $f_{k}$ take $x_{k}$ to 1 and let $g_{k}$ take $x_{k}$ to $x_{i}$; let $f_{i}$ take $x_{i}{ }^{2}$ to 1 and let $g_{i}$ take $x_{i}{ }^{2}$ to $x_{i}$. It is easy to check that:

Proposition 2.1.1. The tangent space $\operatorname{Hom}_{R}\left(I_{i}, R / I_{i}\right)$ to $\operatorname{Hilb}^{2} \mathbb{A}^{r}$ at the fixed point $I_{i}$ has dimension $2 r$, having the set $\left\{f_{k}, g_{k}: 1 \leq k \leq r\right\}$ as a weight basis.

Proposition 2.1.2. If the action of $\mathbb{C}^{*}$ on $R$ defined in (2.0.1) is such that $0<\mu_{1}<\mu_{2}<\cdots<\mu_{r}$ and $\frac{\mu_{j}}{\mu_{i}} \gg 0$ whenever $j>i$, then the part of $\operatorname{Hom}_{R}\left(I_{i}, R / I_{i}\right)$ where the weights of $\mathbb{C}^{*}$ are positive has dimension $i-1$.

Proof. We have only to count the number of basis elements of $\operatorname{Hom}_{R}\left(I_{i}, R / I_{i}\right)$ described in Proposition 2.1.1 where the weights of $\mathbb{C}^{*}$ are positive. In fact, the weight of $\mathbb{C}^{*}$ is positive exactly at the $g_{k}$ s for which $k \leq i-1$.

\subsection{The tangent spaces at the fixed points of $\mathbf{H i l b}^{3} \mathbb{A}^{r}$.}

For $1 \leq i \leq r$, let $\tilde{I}_{i}$ be the ideal of $R$ generated by $x_{i}{ }^{3}$ and the $x_{k}$ s such that $1 \leq \bar{k} \leq r$ and $k \neq i$; for $1 \leq i<j \leq r$, let $\hat{I}_{i, j}$ be the ideal generated by $x_{i}{ }^{2}, x_{j}{ }^{2}, x_{i} x_{j}$ and the $x_{k}$ s such that $1 \leq k \leq r, k \neq i$ and $k \neq j$. The $\tilde{I}_{i}$ s and 
the $\hat{I}_{i, j}$ s are exactly the fixed points of $\operatorname{Hilb}^{3} \mathbb{A}^{r}$ and as before, we will refer to the generators described above for each of these ideals as its canonical generators.

First, let us look at the tangent space $\operatorname{Hom}_{R}\left(\tilde{I}_{i}, R / \tilde{I}_{i}\right)$ to $\operatorname{Hilb}^{3} \mathbb{A}^{r}$ at the fixed point $\tilde{I}_{i}$. We can describe $3 r$ elements $\tilde{f}_{1}, \tilde{f}_{2}, \ldots, \tilde{f}_{r}, \tilde{g}_{1}, \tilde{g}_{2}, \ldots, \tilde{g}_{r}, \tilde{h}_{1}$, $\tilde{h}_{2}, \ldots, \tilde{h}_{r}$ of $\operatorname{Hom}_{R}\left(\tilde{I}_{i}, R / \tilde{I}_{i}\right)$ which take all but one of the canonical generators of $\tilde{I}_{i}$ to zero. If $k \neq i$, let $\tilde{f}_{k}$ take $x_{k}$ to 1 , let $\tilde{g}_{k}$ take $x_{k}$ to $x_{i}$ and let $\tilde{h}_{k}$ take $x_{k}$ to $x_{i}{ }^{2}$; let $\tilde{f}_{i}$ take $x_{i}{ }^{3}$ to 1 , let $\tilde{g}_{i}$ take $x_{i}{ }^{3}$ to $x_{i}$ and let $\tilde{h}_{i}$ take $x_{i}{ }^{3}$ to $x_{i}{ }^{2}$. Again, we check that:

Proposition 2.2.1. The tangent space $\operatorname{Hom}_{R}\left(\tilde{I}_{i}, R / \tilde{I}_{i}\right)$ to $\operatorname{Hilb}^{3} \mathbb{A}^{r}$ at the fixed point $\tilde{I}_{i}$ has dimension $3 r$, having the set $\left\{\tilde{f}_{k}, \tilde{g}_{k}, \tilde{h}_{k}: 1 \leq k \leq r\right\}$ as a weight basis.

Proposition 2.2.2. If the action of $\mathbb{C}^{*}$ on $R$ defined in (2.0.1) is such that $0<\mu_{1}<\mu_{2}<\cdots<\mu_{r}$ and $\frac{\mu_{j}}{\mu_{i}} \gg 0$ whenever $j>i$, then the part of $\operatorname{Hom}_{R}\left(\tilde{I}_{i}, R / \tilde{I}_{i}\right)$ where the weights of $\mathbb{C}^{*}$ are positive has dimension $2(i-1)$.

Proof. The weight of $\mathbb{C}^{*}$ is positive exactly at the $\tilde{g}_{k}$ s for which $k \leq i-1$ and at the $\tilde{h}_{k}$ s for which $k \leq i-1$.

We now turn our attention to the tangent space $\operatorname{Hom}_{R}\left(\hat{I}_{i, j}, R / \hat{I}_{i, j}\right)$ to Hilb $^{3} \mathbb{A}^{r}$ at the fixed point $\hat{I}_{i, j}$. As before, let us describe $3 r$ elements $\hat{f}_{1}, \hat{f}_{2}, \ldots, \hat{f}_{r}, \hat{g}_{1}, \hat{g}_{2}, \ldots, \hat{g}_{r}, \hat{h}_{1}, \hat{h}_{2}, \ldots, \hat{h}_{r}$ of $\operatorname{Hom}_{R}\left(\hat{I}_{i, j}, R / \hat{I}_{i, j}\right)$ which take all but one of the canonical generators of $\hat{I}_{i, j}$ to zero. If $1 \leq k \leq r, k \neq i$ and $k \neq j$, let $\hat{f}_{k}$ take $x_{k}$ to 1 , let $\hat{g}_{k}$ take $x_{k}$ to $x_{i}$ and let $\hat{h}_{k}$ take $x_{k}$ to $x_{j}$; let $\hat{g}_{i}$ take $x_{i}{ }^{2}$ to $x_{i}$, let $\hat{h}_{i}$ take $x_{i}{ }^{2}$ to $x_{j}$, let $\hat{g}_{j}$ take $x_{j}{ }^{2}$ to $x_{i}$, let $\hat{h}_{j}$ take $x_{j}{ }^{2}$ to $x_{j}$, let $\hat{f}_{i}$ take $x_{i} x_{j}$ to $x_{j}$ and let $\hat{f}_{j}$ take $x_{i} x_{j}$ to $x_{i}$. Once more, we can verify that:

Proposition 2.2.3. The tangent space $\operatorname{Hom}_{R}\left(\hat{I}_{i, j}, R / \hat{I}_{i, j}\right)$ to $\operatorname{Hilb}^{3} \mathbb{A}^{r}$ at the fixed point $\hat{I}_{i, j}$ has dimension $3 r$, having the set $\left\{\hat{f}_{k}, \hat{g}_{k}, \hat{h}_{k}: 1 \leq k \leq r\right\}$ as a weight basis.

Proposition 2.2.4. If the action of $\mathbb{C}^{*}$ on $R$ defined in (2.0.1) is such that $0<\mu_{1}<\mu_{2}<\cdots<\mu_{r}$ and $\frac{\mu_{j}}{\mu_{i}} \gg 0$ whenever $j>i$, then the part of $\operatorname{Hom}_{R}\left(\hat{I}_{i, j}, R / \hat{I}_{i, j}\right)$ where the weights of $\mathbb{C}^{*}$ are positive has dimension $i+j-2$.

Proof. The weight of $\mathbb{C}^{*}$ is positive exactly at the $\hat{g}_{k}$ s for which $k \leq i-1$ and at the $\hat{h}_{k}$ s for which $k \leq j-1$. 


\subsection{The tangent spaces at the fixed points of $Z_{1,2}\left(\mathbb{A}^{r}\right)$.}

Let $M$ be the maximal ideal of $R$ generated by all the $x_{k}$ 's for which $1 \leq k \leq r$ and let $I_{i}$ be the ideal described in Section 2.1. There are exactly $r$ fixed points on $Z_{1,2}\left(\mathbb{A}^{r}\right)$, the $i^{t h}$ fixed point being the pair of ideals $\left(M, I_{i}\right)$.

For $1 \leq k \leq r$, let $\tilde{\tilde{f}}_{k} \in \operatorname{Hom}_{R}(M, R / M)$ take $x_{k}$ to 1 and all the other $x_{j}$ s to zero. Also, let $f_{k}$ and $g_{k} \in \operatorname{Hom}_{R}\left(I_{i}, R / I_{i}\right)$ be the homomorphisms described in Section 2.1. As usual, we check that:

Proposition 2.3.1. The tangent space to $Z_{1,2}\left(\mathbb{A}^{r}\right)$ at the fixed point $\left(M, I_{i}\right)$ has dimension $2 r$, having the set $\left\{\left(0, g_{k}\right): 1 \leq k \leq r\right\} \cup\left\{\left(\tilde{\tilde{f}}_{k}, f_{k}\right): 1 \leq k \leq\right.$ $r, k \neq i\} \cup\left\{\left(\tilde{\tilde{f}}_{i}, 0\right)\right\}$ as a weight basis.

Proposition 2.3.2. If the action of $\mathbb{C}^{*}$ on $R$ defined in (2.0.1) is such that $0<\mu_{1}<\mu_{2}<\cdots<\mu_{r}$ and $\frac{\mu_{j}}{\mu_{i}} \gg 0$ whenever $j>i$, then the part of the tangent space to $Z_{1,2}\left(\mathbb{A}^{r}\right)$ at the fixed point $\left(M, I_{i}\right)$ where the weights of $\mathbb{C}^{*}$ are positive has dimension $i-1$.

Proof. The weight of $\mathbb{C}^{*}$ is positive exactly at the $\left(0, g_{k}\right)$ s for which $k \leq$ $i-1$.

\subsection{The tangent spaces at the fixed points of $Z_{2,3}\left(\mathbb{A}^{r}\right)$.}

With the notation of Sections 2.1 and 2.2, the fixed points of $Z_{2,3}\left(\mathbb{A}^{r}\right)$ are the pairs of ideals $\left(I_{i}, \tilde{I}_{i}\right)$ for which $1 \leq i \leq r$, the pairs of ideals $\left(I_{i}, \hat{I}_{i, j}\right)$ for which $1 \leq i<j \leq r$ and the pairs of ideals $\left(I_{j}, \hat{I}_{i, j}\right)$ for which $1 \leq i<j \leq r$. As always, it is tedious but straightforward enough to verify the following:

Proposition 2.4.1. The tangent space to $Z_{2,3}\left(\mathbb{A}^{r}\right)$ at $\left(I_{i}, \tilde{I}_{i}\right)$ has dimension $3 r$ and is the subspace of $\operatorname{Hom}_{R}\left(I_{i}, R / I_{i}\right) \oplus \operatorname{Hom}_{R}\left(\tilde{I}_{i}, R / \tilde{I}_{i}\right)$ spanned by $\left(f_{i}, \tilde{g}_{i}\right)$, $\left(g_{i}, 0\right)$, the $\left(f_{k}, \tilde{f}_{k}\right) s$ for which $k \neq i$, the $\left(g_{k}, \tilde{g}_{k}\right) s$ for which $k \neq i$ and all of the $\left(0, \tilde{h}_{k}\right) s$.

Proposition 2.4.2. If the action of $\mathbb{C}^{*}$ on $R$ defined in (2.0.1) is such that $0<\mu_{1}<\mu_{2}<\cdots<\mu_{r}$ and $\frac{\mu_{j}}{\mu_{i}} \gg 0$ whenever $i<j$, then the part of the tangent space to $Z_{2,3}\left(\mathbb{A}^{r}\right)$ at the fixed point $\left(I_{i}, \tilde{I}_{i}\right)$ where the weights of $\mathbb{C}^{*}$ are positive has dimension $2(i-1)$.

Proof. The weight of $\mathbb{C}^{*}$ is positive exactly at the $\left(g_{k}, \tilde{g}_{k}\right)$ s for which $k \leq i-1$ and at the $\left(0, \tilde{h}_{k}\right)$ s for which $k \leq i-1$.

One checks easily that:

Proposition 2.4.3. The tangent space to $Z_{2,3}\left(\mathbb{A}^{r}\right)$ at $\left(I_{i}, \hat{I}_{i, j}\right)$ has dimension $3 r$ and is the subspace of $\operatorname{Hom}_{R}\left(I_{i}, R / I_{i}\right) \oplus \operatorname{Hom}_{R}\left(\hat{I}_{i, j}, R / \hat{I}_{i, j}\right)$ spanned 
by $\left(0, \hat{f}_{i}\right),\left(g_{j}, 0\right)$, the $\left(f_{k}, \hat{f}_{k}\right)$ s for which $k \neq i$, the $\left(g_{k}, \hat{g}_{k}\right)$ s for which $k \neq j$ and all of the $\left(0, \hat{h}_{k}\right) s$; the tangent space to $Z_{2,3}\left(\mathbb{A}^{r}\right)$ at $\left(I_{j}, \hat{I}_{i, j}\right)$ has dimension $3 r$ and is the subspace of $\operatorname{Hom}_{R}\left(I_{j}, R / I_{j}\right) \oplus \operatorname{Hom}_{R}\left(\hat{I}_{i, j}, R / \hat{I}_{i, j}\right)$ spanned by $\left(0, \hat{f}_{j}\right),\left(g_{i}, 0\right)$, the $\left(f_{k}, \hat{f}_{k}\right)$ s for which $k \neq j$, the $\left(g_{k}, \hat{g}_{k}\right) s$ for which $k \neq i$ and all of the $\left(0, \hat{h}_{k}\right) s$.

Proposition 2.4.4. If the action of $\mathbb{C}^{*}$ on $R$ defined in (2.0.1) is such that $0<\mu_{1}<\mu_{2}<\cdots<\mu_{r}$ and $\frac{\mu_{j}}{\mu_{i}} \gg 0$ whenever $i<j$, then:

(1) The part of the tangent space to $Z_{2,3}\left(\mathbb{A}^{r}\right)$ at the fixed point $\left(I_{i}, \hat{I}_{i, j}\right)$ where the weights of $\mathbb{C}^{*}$ are positive has dimension $i+j-2$.

(2) The part of the tangent space to $Z_{2,3}\left(\mathbb{A}^{r}\right)$ at the fixed point $\left(I_{j}, \hat{I}_{i, j}\right)$ where the weights of $\mathbb{C}^{*}$ are positive has dimension $i+j-2$.

Proof. (1): The weight of $\mathbb{C}^{*}$ is positive exactly at the $\left(g_{k}, \hat{g}_{k}\right)$ 's for which $k \leq i-1$ and at the $\left(0, \hat{h}_{k}\right)$ 's for which $k \leq j-1$.

(2): The weight of $\mathbb{C}^{*}$ is positive exactly at $\left(g_{i}, 0\right)$, at the $\left(0, \hat{h}_{k}\right)$ s for which $k \leq i-1$ and at the $\left(g_{k}, \hat{g}_{k}\right)$ s for which $k \leq j-1$ and $k \neq i$.

\subsection{The tangent spaces at the fixed points of Hilb $\mathbb{A}^{2}$.}

In the next couple of sections, we will be dealing with the case $r=2$. To avoid a proliferation of subscripts, we denote $x_{1}$ by $x$ and $x_{2}$ by $y$.

If the ideal $I$ corresponds to a $\mathbb{C}^{*}$-fixed point of $\mathrm{Hilb}^{n} \mathbb{A}^{2}$, it is generated by monomials and we can represent it by a Young diagram with $n$ boxes whose $i^{\text {th }}$ row has $\min \left\{j: y^{i} x^{j} \in I\right\}$ boxes. In fact, this gives us a one-to-one correspondence between the fixed points of Hilb $\mathbb{A}^{2}$ and the set of Young diagrams with $n$ boxes.

Example. The ideal $I=\left\langle x^{5}, y x^{4}, y^{4} x^{2}, y^{6}\right\rangle$ is represented by the diagram

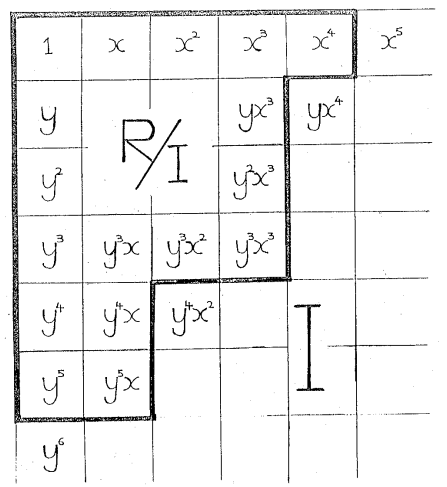

Figure 1. 
As in the example above, we can regard the boxes of our Young diagram as a subset of the boxes representing $\mathbb{N} \times \mathbb{N}$, with the box representing a pair $(i, j) \in \mathbb{N} \times \mathbb{N}$ containing the monomial $y^{i} x^{j}$, so that monomials outside the Young diagram are in $I$ and monomials in the Young diagram form a basis of $R / I$ over $\mathbb{C}$.

If we look at the Young diagram of an invariant ideal $I$, the "corners" of its complement, that is the shaded boxes in the picture above, represent the unique minimal set of monomials that generate $I$ and we will call them the "canonical generators".

To describe an $R$-homomorphism $f: I \rightarrow R / I$, we need only specify the elements to which $f$ takes each of the canonical generators. Since $\mathbb{C}^{*}$ acts on $\operatorname{Hom}_{R}(I, R / I)$, it should be possible to find a weight basis of $\operatorname{Hom}_{R}(I, R / I)$. For $f \in \operatorname{Hom}_{R}(I, R / I)$ to be an element of pure weight, $f$ must take each canonical generator to a scalar multiple of some monomial modulo $I$.

Let us first look at the subset $\mathcal{S}$ of $\operatorname{Hom}_{R}(I, R / I)$ consisting of elements of pure weight which take canonical generators either to zero or to monomials modulo $I$. If $A$ denotes the set of canonical generators of $I$ and $B$ denotes the set of monomials not in $I$, then every element $f \in \mathcal{S}$ is specified by a map from $A$ to $B$.
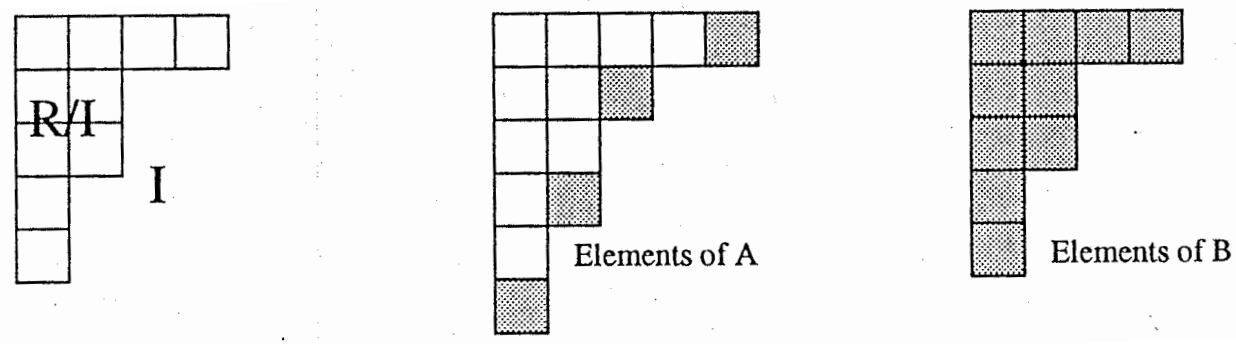

Figure 2.

In fact, since $f \in \mathcal{S}$ is required to have pure weight, it moves any two canonical generators in $A$ which it does not take to zero by the same horizontal and the same vertical translations.

If $\alpha \in A$ and $\beta \in B$, let $S_{\alpha, \beta}=\{f \in \mathcal{S}: f(\alpha)=\beta\}$. The set $S_{\alpha, \beta}$ could well be empty since although every map from $A$ to $B$ can be extended to a $\mathbb{C}$-linear map from $I$ to $R / I$, not every map specifies an $R$-module homomorphism. For example, if $I=\left\langle x^{3}, y^{2} x, y^{4}\right\rangle$, the set $S_{x^{3}, y}$ is empty. When $S_{\alpha, \beta}$ is not empty, let $f_{\alpha, \beta}$ be the unique homomorphism in $S_{\alpha, \beta}$ which takes the largest number of canonical generators to zero. 
We wish to construct a basis of $\operatorname{Hom}_{R}(I, R / I)$ consisting of elements of the form $f_{\alpha, \beta}$. First, we have to set up yet more notation.

Let us label the canonical generators $\alpha_{0}, \alpha_{1}, \alpha_{2}, \ldots, \alpha_{m}$ as we go down the picture and for $0 \leq j \leq m-1$, let $p_{j}$ denote the vertical distance between $\alpha_{j}$ and $\alpha_{j+1}$ while for $1 \leq j \leq m$, let $q_{j}$ denote the horizontal distance between $\alpha_{j}$ and $\alpha_{j-1}$.

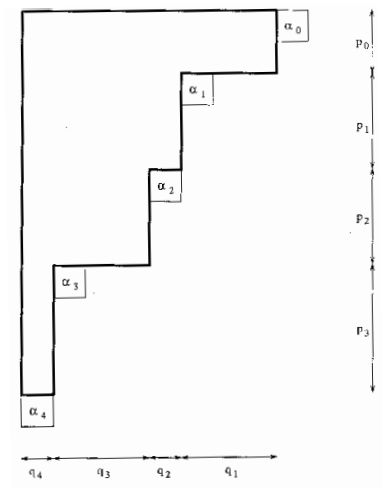

Figure 3.

For any $\alpha=\alpha_{t} \in A$, let $P_{\alpha}$ be the subset of $B$ which consists of the elements $b$ satisfying

(1) $b$ lies to the left of $\alpha$,

(2) $y^{p_{t}} b \in I$,

and let $Q_{\alpha}$ be the subset of $B$ which consists of the elements $b$ satisfying

(1) $b$ lies above $\alpha$,

(2) $x^{q_{t}} b \in I$.

Example. For the ideal $I=\left\langle x^{5}, y x^{4}, y^{4} x^{2}, y^{6}\right\rangle$, we have

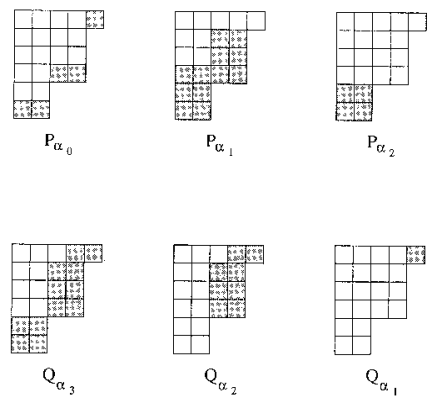

Figure 4. 
Lemma 2.5.1. If $\beta \in P_{\alpha}$ or $\beta \in Q_{\alpha}$, then $S_{\alpha, \beta} \neq \emptyset$.

Proof. In fact, let us explicitly construct the homomorphism $f_{\alpha, \beta}$ defined earlier.

Suppose first that $\beta \in P_{\alpha}$. This means that $\beta$ must lie $q$ squares to the left and $p$ squares below $\alpha=\alpha_{t}$ for some natural numbers $p$ and $q$, that is to say $\beta=\alpha\left(\frac{y^{p}}{x^{q}}\right)$. If we let

$$
l=\max \left\{i: 0 \leq i \leq t, \quad \alpha_{i}\left(\frac{y^{p}}{x^{q}}\right) x^{q_{i}} \in I\right\},
$$

then $f_{\alpha, \beta} \in \mathcal{S}$ is given by

$$
f_{\alpha, \beta}\left(\alpha_{i}\right)= \begin{cases}0 & \text { if } 0 \leq i<l \\ \alpha_{i}\left(\frac{y^{p}}{x^{q}}\right) & \text { if } l \leq i \leq t \\ \beta & \text { if } i=t \\ 0 & \text { if } t<i\end{cases}
$$

Next, suppose that $\beta \in Q_{\alpha}$. Then, $\beta$ must lie $q$ squares to the right and $p$ squares above $\alpha=\alpha_{t}$ for some natural numbers $p$ and $q$ or equivalently, $\beta=\alpha\left(\frac{x^{q}}{y^{p}}\right)$. If we let

$$
l=\min \left\{i: 0 \leq i \leq t, \quad \alpha_{i}\left(\frac{x^{q}}{y^{p}}\right) y^{p_{i}} \in I\right\}
$$

then $f_{\alpha, \beta} \in \mathcal{S}$ is given by

$$
f_{\alpha, \beta}\left(\alpha_{i}\right)= \begin{cases}0 & \text { if } 0 \leq i<t \\ \beta & \text { if } i=t, \\ \alpha_{i}\left(\frac{x^{q}}{y^{p}}\right) & \text { if } t \leq i \leq l, \\ 0 & \text { if } l<i .\end{cases}
$$

Lemma 2.5.2. If $\beta \in P_{\alpha} \cup Q_{\alpha}$ and $\beta^{\prime} \in P_{\alpha^{\prime}} \cup Q_{\alpha^{\prime}}, f_{\alpha, \beta}=f_{\alpha^{\prime}, \beta^{\prime}}$ implies that $(\alpha, \beta)=\left(\alpha^{\prime}, \beta^{\prime}\right)$.

Proof. Suppose that $\beta \in P_{\alpha}$. Then, $\beta$ must be situated to the left of $\alpha$. Also, if $f_{\alpha, \beta}\left(\alpha^{\prime}\right)$ is not zero, its position relative to $\alpha^{\prime}$ is the same as the position of $\beta$ relative to $\alpha$. Since $f_{\alpha, \beta}=f_{\alpha^{\prime}, \beta^{\prime}}$, we know in our case that $f_{\alpha, \beta}\left(\alpha^{\prime}\right)=f_{\alpha^{\prime}, \beta^{\prime}}\left(\alpha^{\prime}\right)=\beta^{\prime}$, so $\beta^{\prime}$ lies to the left of $\alpha^{\prime}$ and $\beta^{\prime}$ must be in $P_{\alpha^{\prime}}$ rather than $Q_{\alpha^{\prime}}$. 
As $\beta \in P_{\alpha}$ and $f_{\alpha, \beta}$ is not zero at $\alpha^{\prime}, \alpha^{\prime}$ cannot lie to the left of $\alpha$. On the other hand, as $\beta^{\prime} \in P_{\alpha^{\prime}}$ and $f_{\alpha^{\prime}, \beta^{\prime}}$ is not zero at $\alpha, \alpha$ cannot lie to the left of $\alpha^{\prime}$. The only possibility is that $\alpha$ and $\alpha^{\prime}$ are in fact one and the same canonical generator. Moreover, $\beta$ and $\beta^{\prime}$ are also equal because $\beta=f_{\alpha, \beta}(\alpha)=f_{\alpha^{\prime}, \beta^{\prime}}(\alpha)=f_{\alpha^{\prime}, \beta^{\prime}}\left(\alpha^{\prime}\right)=\beta^{\prime}$.

An analogous argument works if we start by supposing that $\beta \in Q_{\alpha}$.

Lemma 2.5.3. The set $\left\{f_{\alpha, \beta}: \alpha \in A\right.$ and $\left.\beta \in P_{\alpha} \cup Q_{\alpha}\right\}$ has $2 n$ elements.

Proof. Let $\|\mathcal{M}\|$ denote the cardinality of the set $\mathcal{M}$. By Lemma 2.5.2, we have

$$
\begin{aligned}
& \|\left\{f_{\alpha, \beta}: \alpha \in A \text { and } \beta \in P_{\alpha} \cup Q_{\alpha}\right\} \| \\
& =\|\left\{(\alpha, \beta): \alpha \in A \text { and } \beta \in P_{\alpha} \cup Q_{\alpha}\right\} \| \\
& =\sum_{\alpha \in A}\left\|\left\{(\alpha, \beta): \beta \in P_{\alpha} \cup Q_{\alpha}\right\}\right\| \\
& =\sum_{\alpha \in A}\left\|P_{\alpha} \cup Q_{\alpha}\right\| .
\end{aligned}
$$

Since $P_{\alpha}$ and $Q_{\alpha}$ are clearly disjoint, $\left\|P_{\alpha} \cup Q_{\alpha}\right\|=\left\|P_{\alpha}\right\|+\left\|Q_{\alpha}\right\|$ and hence,

$$
\|\left\{f_{\alpha, \beta}: \alpha \in A \text { and } \beta \in P_{\alpha} \cup Q_{\alpha}\right\}\left\|=\sum_{\alpha \in A}\right\| P_{\alpha}\left\|+\sum_{\alpha \in A}\right\| Q_{\alpha} \|=n+n=2 n .
$$

Proposition 2.5.4. The tangent space $\operatorname{Hom}_{R}(I, R / I)$ to $\operatorname{Hilb}^{n} \mathbb{A}^{2}$ at the fixed point $I$ is of dimension $2 n$, having the set $\mathcal{T}=\left\{f_{\alpha, \beta}: \alpha \in A\right.$ and $\beta \in$ $\left.P_{\alpha} \cup Q_{\alpha}\right\}$ as a weight basis.

Comment. Though the first part of the proposition is well-known [Fo], we give an independent proof here.

Proof. First, we show that the homomorphisms $f_{\alpha, \beta}$ in the set $\mathcal{T}$ are linearly independent. Assume for contradiction that there exists linearly dependent elements in the set $\mathcal{T}$. Then, there must exist linearly dependent elements of the same weight and we can write

$$
f_{\alpha_{i_{1}}, \beta_{i_{1}}}+\lambda_{2} f_{\alpha_{i_{2}}, \beta_{i_{2}}}+\cdots+\lambda_{r} f_{\alpha_{i_{r}}, \beta_{i_{r}}}=0
$$

Either every $\beta_{i_{j}}$ is in $P_{\alpha_{i_{j}}}$ or every $\beta_{i_{j}}$ is in $Q_{\alpha_{i_{j}}}$. Let us suppose that the first case holds since the argument needed if the second case holds is, as always, exactly analogous. We may also assume that $i_{1}>i_{2}>\cdots>i_{r}$. Because $f_{\alpha_{i_{j}}, \beta_{i_{j}}}\left(\alpha_{i_{1}}\right)=0$ for all $j \geq 2$, Equation (2.5.1) implies that $f_{\alpha_{i_{1}}, \beta_{i_{1}}}\left(\alpha_{i_{1}}\right)=0$ 
but this is a contradiction since we know that $f_{\alpha_{i_{1}}, \beta_{i_{1}}}\left(\alpha_{i_{1}}\right)=\beta_{i_{1}}$ from the definition of $f_{\alpha_{i_{1}}, \beta_{i_{1}}}$.

It only remains to show that the set $\mathcal{T}$ indeed spans $\operatorname{Hom}_{R}(I, R / I)$. As $\operatorname{Hom}_{R}(I, R / I)$ is spanned by homomorphisms of pure weight, we need only show that any $f \in \operatorname{Hom}_{R}(I, R / I)$ of pure weight is in the span of $\mathcal{T}$. We argue by induction on the number $n(f)$ of canonical generators not taken by $\mathrm{f}$ to zero. The base step of the induction is trivial because if $n(f)=0$, then $f$ is just the zero-homomorphism.

Now, suppose that we are given $f \in \operatorname{Hom}_{R}(I, R / I)$ of pure weight with $n(f)>0$ and that any $g \in \operatorname{Hom}_{R}(I, R / I)$ of pure weight with $n(g)<n(f)$ is known to be in the span of $\mathcal{T}$. Since $f$ is of pure weight, we know that either

(1) $r_{1}=\max \left\{i: f\left(\alpha_{i}\right) \neq 0\right\}$ is less than $m$ and a scalar multiple of $f\left(\alpha_{r_{1}}\right)$ is represented by an element of $B$ to the left of $\alpha_{r_{1}}$ or

(2) $r_{2}=\min \left\{i: f\left(\alpha_{i}\right) \neq 0\right\}$ is greater than zero and a scalar multiple of $f\left(\alpha_{r_{2}}\right)$ is represented by an element of $B$ above $\alpha_{r_{2}}$.

In the first case, we may assume without loss of generality that $f\left(\alpha_{r_{1}}\right)$ is a monomial and since $f\left(\alpha_{r_{1}+1}\right)=0$, we know that $y^{p_{r_{1}}} f\left(\alpha_{r_{1}}\right) \in I$ and $f\left(\alpha_{r_{1}}\right) \in$ $P_{\alpha_{r_{1}}}$. This means that $f_{\alpha_{r_{1}}, f\left(\alpha_{r_{1}}\right)}$ is in $\mathcal{T}$. Moreover, since $\left(f-f_{\alpha_{r_{1}}, f\left(\alpha_{r_{1}}\right)}\right)$ is an element of pure weight satisfying $n\left(f-f_{\alpha_{r_{1}}, f\left(\alpha_{r_{1}}\right)}\right)<n(f)$, it is in the span of $\mathcal{T}$ and consequently, so too is $f$.

Not surprisingly, the second case where $r_{2}$ is greater than zero and a scalar multiple of $f\left(\alpha_{r_{2}}\right)$ lies above $\alpha_{r_{2}}$ can be dealt with in a completely analogous way.

Proposition 2.5.5 (Ellingsrud-Strømme). If the action of $\mathbb{C}^{*}$ on $R=$ $\mathbb{C}[x, y]$ defined in (2.0.1) is such that $0<\mu_{1}<\mu_{2}$ and $\frac{\mu_{2}}{\mu_{1}} \gg 0$, the part of the tangent space $T$ to $\mathrm{Hilb}^{n} \mathbb{A}^{2}$ at the fixed point I where the weights of $\mathbb{C}^{*}$ are positive has dimension $(n-d)$ where $d=\min \left\{i: x^{i} \in I\right\}$.

Proof. The weight of $\mathbb{C}^{*}$ is positive at the $f_{\alpha, \beta}$ for which $\beta \in P_{\alpha}$ and $\beta$ lies on a lower row than $\alpha$. Thus, if we let $R_{\alpha}$ denote the subset of $P_{\alpha}$ consisting of monomials lying on the same row as $\alpha$, we have

$$
\operatorname{dim} T^{+}=\sum_{\alpha \in A}\left\|P_{\alpha}\right\|-\sum_{\alpha \in A}\left\|R_{\alpha}\right\|=n-\sum_{\alpha \in A}\left\|R_{\alpha}\right\|
$$

and it only remains to show that $\sum_{\alpha \in A}\left\|R_{\alpha}\right\|=d$. Since $R_{\alpha_{i}}$ consists of the $q_{i+1}$ elements to the left of $\alpha_{i}, \sum_{\alpha \in A}\left\|R_{\alpha}\right\|=\sum_{i=0}^{m} q_{i+1}=d$ as required. (See picture below.) 


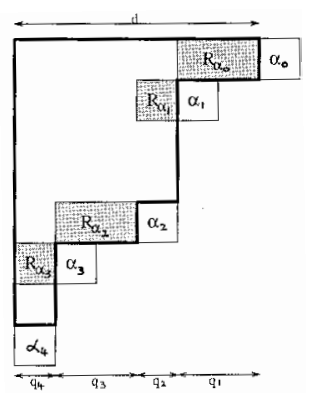

Figure 5.

\subsection{The tangent spaces at the fixed points of $Z_{n-1, n}\left(\mathbb{A}^{2}\right)$.}

Suppose $\left(I_{1}, I_{2}\right)$ is a fixed point of $Z_{n-1, n}\left(\mathbb{A}^{2}\right), I_{1}$ is a fixed point of $\mathrm{Hilb}^{n-1} \mathbb{A}^{2}$ and $I_{2}$ is a fixed point of $\mathrm{Hilb}^{n} \mathbb{A}^{2}$, so we obtain as in Section 2.5 a pair of Young diagrams, one sitting inside the other.
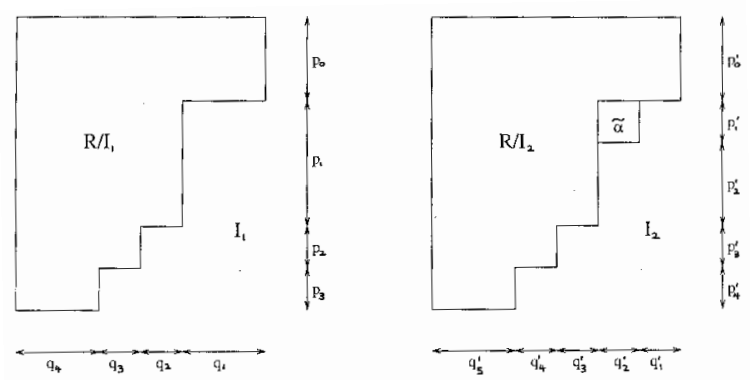

\section{Figure 6.}

Let $B_{1}$ denote the set of monomials not in $I_{1}$, let $B_{2}$ denote the set of monomials not in $I_{2}$, let $A_{1}=\left\{\alpha_{0}, \alpha_{1}, \ldots, \alpha_{m}\right\}$ be the canonical generators of $I_{1}$ and let $A_{2}=\left\{\alpha_{0}^{\prime}, \alpha_{1}^{\prime}, \ldots, \alpha_{s}^{\prime}\right\}$ be the canonical generators of $I_{2}$. As before, generators which involve higher powers of $x$ come earlier in the list. Since the ideal $I_{1}$ has length $n-1$ and the ideal $I_{2}$ has length $n$, the Young diagram of $I_{2}$ consists of adding to the Young diagram of $I_{1}$ one of the boxes which represents a canonical generator of $I_{1}$. Let us suppose that $\alpha_{k}$ is this monomial which is contained in $I_{1}$ and not $I_{2}$. There are essentially three different cases:

Case 1. $p_{k}=1$ or $q_{k}=1$ but not both.

Here, $s=m, \alpha_{k}^{\prime} \notin A_{1}$ and $\alpha_{i}^{\prime}=\alpha_{i}$ if $0 \leq i \leq s$ and $i \neq k$. In some of the proofs to come, we have to subdivide this case into two cases, the Case 1a where $q_{k}=1$ and the Case $1 \mathrm{~b}$ where $p_{k}=1$. 
Case 2. $\left(k=0, p_{0}>1\right)$ or $\left(k=m, q_{m}>1\right)$ or $\left(0<k<m, p_{k}>1, q_{k}>1\right)$.

Here, $s=m+1, \alpha_{k}^{\prime}$ and $\alpha_{k+1}^{\prime}$ are not in $A_{1}, \alpha_{i}^{\prime}=\alpha_{i}$ if $0 \leq i \leq k-1$ and $\alpha_{i}^{\prime}=\alpha_{i-1}$ if $k+2 \leq i \leq s$.

Case 3. Both $p_{k}=1$ and $q_{k}=1$.

Here, $s=m-1, \alpha_{i}^{\prime}=\alpha_{i}$ if $0 \leq i \leq k-1$ and $\alpha_{i}^{\prime}=\alpha_{i+1}$ if $k \leq i \leq s$.

Recall from Section 0.4 that $\operatorname{Ker}(\phi-\psi)$ is the tangent space to $Z_{n-1, n}\left(\mathbb{A}^{2}\right)$ at $\left(I_{1}, I_{2}\right)$. Each of the maps $\phi, \psi$ and $(\phi-\psi)$ preserves the action of $\mathbb{C}^{*}$ on the spaces involved.

Let $\mathcal{W}$ be the subset of $\operatorname{Hom}_{R}\left(I_{2}, R / I_{1}\right)$ consisting of the elements of pure weight which take the canonical generators of $I_{2}$ either to zero or to monomials in $B_{1}$. Then,

Lemma 2.6.1. The vector space $\operatorname{Hom}_{R}\left(I_{2}, R / I_{1}\right)$ is generated by the elements in $\mathcal{W}$.

Proof. Since $\operatorname{Hom}_{R}\left(I_{2}, R / I_{1}\right)$ has a weight basis, it can be generated by elements of pure weight, so we need only show that any $f \in \operatorname{Hom}_{R}\left(I_{2}, R / I_{1}\right)$ of pure weight is in the span of $\mathcal{W}$. We know that $f$ necessarily takes the generators of $I_{2}$ to scalar multiples of the monomials in $B_{1}$. As in Proposition 2.5.4, we argue by induction on the number $n(f)$ of canonical generators in $A_{2}$ not taken by $f$ to zero. The base step of the induction is trivial because $n(f)=0$ implies that $f$ is just the zero-homomorphism.

Now, suppose that we are given $f \in \operatorname{Hom}_{R}\left(I_{2}, R / I_{1}\right)$ of pure weight with $n(f)>0$ and that any $g \in \operatorname{Hom}_{R}\left(I_{2}, R / I_{1}\right)$ of pure weight with $n(g)<n(f)$ is known to be in the span of $\mathcal{W}$. If $r_{1}=\max \left\{i: f\left(\alpha_{i}^{\prime}\right) \neq 0\right\}$ and $r_{2}=$ $\min \left\{i: f\left(\alpha_{i}^{\prime}\right) \neq 0\right\}$, one can check that either

(1) $r_{1}<s$ and a scalar multiple of $f\left(\alpha_{r_{1}}^{\prime}\right)$ is represented by an element of $B_{1}$ to the left of $\alpha_{r_{1}}^{\prime}$ or

(2) $\quad r_{2}>0$ and a scalar multiple of $f\left(\alpha_{r_{2}}^{\prime}\right)$ is represented by an element of $B_{1}$ above $\alpha_{r_{2}}^{\prime}$.

Let us consider just the first case since the other case can be dealt with in exactly the same way. By multiplying $f$ by a scalar if necessary, assume that $f\left(\alpha_{r_{1}}^{\prime}\right)$ is represented by a monomial in $B_{1}$. Since $f\left(\alpha_{r_{1}+1}^{\prime}\right)=0, f\left(\alpha_{r_{1}}^{\prime}\right) y^{p_{r_{1}}}$ is in the ideal $I_{1}$ and $f\left(\alpha_{r_{1}}^{\prime}\right)$ does not lie in a higher row than $\alpha_{r_{1}}^{\prime}$ unless $k=r_{1}+1, x \alpha_{r_{1}+1}^{\prime}=y^{p_{r_{1}}^{\prime}} \alpha_{r_{1}}^{\prime}, \alpha_{r_{1}+1}^{\prime}=y \alpha_{r_{1}+1}$ and $x y f\left(\alpha_{r_{1}}^{\prime}\right)=\alpha_{r_{1}}^{\prime}$. We can easily check that the second situation cannot arise, so $f\left(\alpha_{r_{1}}^{\prime}\right)=\alpha_{r_{1}}^{\prime}\left(\frac{y^{p}}{x^{q}}\right)$ for some natural numbers $p$ and $q$.

Let $l=\max \left\{i: 0 \leq i \leq r_{1}, \quad \alpha_{i}^{\prime}\left(\frac{y^{p}}{x^{q}}\right) x^{q_{i}} \in I_{1}\right\}$ and define $\tilde{f} \in$ 
$\operatorname{Hom}_{R}\left(I_{2}, R / I_{1}\right)$ by

$$
\tilde{f}\left(\alpha_{i}^{\prime}\right)= \begin{cases}0 & \text { if } 0 \leq i<l, \\ \alpha_{i}^{\prime}\left(\frac{y^{p}}{x^{q}}\right) & \text { if } l \leq i \leq r_{1}, \\ f\left(\alpha_{r_{1}}^{\prime}\right) & \text { if } i=r_{1}, \\ 0 & \text { if } r_{1}<i \leq s\end{cases}
$$

Clearly, $\tilde{f}$ is in the span of $\mathcal{W}$ and so too is $(f-\tilde{f})$ since it has pure weight and satisfies $n(f-\tilde{f})<n(f)$. Therefore, $f$ itself is in the span of $\mathcal{W}$ as needed.

Lemma 2.6.2. The map

$$
(\phi-\psi): \operatorname{Hom}_{R}\left(I_{1}, R / I_{1}\right) \oplus \operatorname{Hom}_{R}\left(I_{2}, R / I_{2}\right) \rightarrow \operatorname{Hom}_{R}\left(I_{2}, R / I_{1}\right)
$$

is surjective.

Proof. As $\operatorname{Im} \phi \subset \operatorname{Im}(\phi-\psi)$ and $\operatorname{Im} \psi \subset \operatorname{Im}(\phi-\psi)$, we need only show that $\operatorname{Im} \phi$ and $\operatorname{Im} \psi$ together generate all of $\operatorname{Hom}_{R}\left(I_{2}, R / I_{1}\right)$. Notice that the elements $f$ of $\mathcal{W}$ which are in $\operatorname{Im} \psi$ are characterized by the property that for all $i, f\left(\alpha_{i}^{\prime}\right)$ is $p_{i}^{\prime}-1$ units above $\alpha_{k}$ if and only if $f\left(\alpha_{i+1}^{\prime}\right)$ is $q_{i+1}^{\prime}-1$ units to the left of $\alpha_{k}$.

Let $\mathcal{U}_{i}$ be the subset of $\mathcal{W}$ consisting of homomorphisms $f$ with the property that $f\left(\alpha_{i+1}^{\prime}\right)$ is $q_{i+1}^{\prime}-1$ units to the left of $\alpha_{k}$ but $f\left(\alpha_{i}^{\prime}\right)$ is zero; let $\mathcal{V}_{i}$ be the subset of $\mathcal{W}$ consisting of homomorphisms $f$ with the property that $f\left(\alpha_{i}^{\prime}\right)$ is $p_{i}^{\prime}-1$ units above $\alpha_{k}$ but $f\left(\alpha_{i+1}^{\prime}\right)$ is zero.

To complete the proof, we have to look at each of the Cases 1a, 1b, 2 and 3 separately.

Case 1a. Here, we have

$$
\begin{array}{cl}
\mathcal{V}_{i} \neq \emptyset & \text { if } 0 \leq i \leq k-2, \\
\mathcal{U}_{i} \neq \emptyset & \text { if } k \leq i \leq s-1 \quad \text { and } \\
\mathcal{V}_{i}=\emptyset \text { and } \mathcal{U}_{i}=\emptyset & \text { if } i=k-1 .
\end{array}
$$

If $0 \leq i \leq k-2$, let $F_{i}$ denote the unique member of $\mathcal{V}_{i}$ which takes the largest number of canonical generators in $A_{2}$ to zero while if $k \leq i \leq s-1$, let $F_{i}$ denote the unique member of $\mathcal{U}_{i}$ which takes the largest number of canonical generators in $A_{2}$ to zero.

One sees easily that all the $F_{i}$ s defined above are linearly independent since they have different weights and it is also not hard to check that any 
element of $\mathcal{W}$ can be generated by $\operatorname{Im} \psi$ together with these $F_{i}$ s. Therefore, we obtain

$$
\operatorname{Hom}_{R}\left(I_{2}, R / I_{1}\right)=\operatorname{Im} \psi \oplus\left(\underset{\substack{i=0 \\ i \neq k-1}}{\bigoplus_{i=1}}\left\langle F_{i}\right\rangle\right) .
$$

Now, observe that if $0 \leq i \leq k-2, F_{i}$ is equal to $\phi\left(f_{\alpha_{i}, \beta}\right)$ for some $\beta \in P_{\alpha_{i}}$ and if $k \leq i \leq s-1, F_{i}$ is equal to $\phi\left(f_{\alpha_{i+1}, \beta}\right)$ for some $\beta \in Q_{\alpha_{i+1}}$. Hence, each of the $F_{i} \mathrm{~s}$ is in $\operatorname{Im} \phi$ and $\operatorname{Hom}_{R}\left(I_{2}, R / I_{1}\right)$ is indeed generated by $\operatorname{Im} \psi$ and $\operatorname{Im} \phi$.

Case 1b. Here, we have

$$
\begin{array}{cl}
\mathcal{V}_{i} \neq \emptyset & \text { if } 0 \leq i \leq k-1, \\
\mathcal{U}_{i} \neq \emptyset & \text { if } k+1 \leq i \leq s-1 \quad \text { and } \\
\mathcal{V}_{i}=\emptyset \text { and } \mathcal{U}_{i}=\emptyset & \text { if } i=k .
\end{array}
$$

With the $F_{i}$ s defined as before, we can again check that

$$
\operatorname{Hom}_{R}\left(I_{2}, R / I_{1}\right)=\operatorname{Im} \psi \oplus\left(\underset{\substack{s-1 \\ \oplus=0 \\ i \neq k}}{i \neq}\left\langle F_{i}\right\rangle\right)
$$

and that the $F_{i}$ s are in $\operatorname{Im} \phi$.

Case 2. Here, we have

$$
\begin{array}{cl}
\mathcal{V}_{i} \neq \emptyset & \text { if } 0 \leq i \leq k-1, \\
\mathcal{U}_{i} \neq \emptyset & \text { if } k+1 \leq i \leq s-1 \quad \text { and } \\
\mathcal{V}_{i}=\emptyset \text { and } \mathcal{U}_{i}=\emptyset & \text { if } i=k .
\end{array}
$$

Once more, we construct homomorphisms $F_{i} \mathrm{~s}$ in $\operatorname{Im} \phi$ so that

$$
\operatorname{Hom}_{R}\left(I_{2}, R / I_{1}\right)=\operatorname{Im} \psi \oplus\left(\underset{\substack{i=0 \\ i \neq k}}{\oplus-1}\left\langle F_{i}\right\rangle\right) .
$$

Case 3. Here, we have

$$
\begin{array}{cl}
\mathcal{V}_{i} \neq \emptyset & \text { if } 0 \leq i \leq k-2, \\
\mathcal{U}_{i} \neq \emptyset & \text { if } k \leq i \leq s-1 \quad \text { and } \\
\mathcal{V}_{i}=\emptyset \text { and } \mathcal{U}_{i}=\emptyset & \text { if } i=k-1 .
\end{array}
$$


So, we repeat the process yet again to obtain homomorphisms $F_{i} \mathrm{~s}$ in $\operatorname{Im} \phi$ with

$$
\operatorname{Hom}_{R}\left(I_{2}, R / I_{1}\right)=\operatorname{Im} \psi \oplus\left(\underset{\substack{i=0 \\ i \neq k-1}}{\bigoplus_{i=1}}\left\langle F_{i}\right\rangle\right)
$$

Proposition 2.6.3. The tangent space $\operatorname{Ker}(\phi-\psi)$ to $Z_{n-1, n}\left(\mathbb{A}^{2}\right)$ at the fixed point $\left(I_{1}, I_{2}\right)$ has dimension $2 n$.

Proof. Denoting the canonical generators of $I_{2}$ by $\alpha_{0}^{\prime}, \alpha_{1}^{\prime}, \ldots, \alpha_{s}^{\prime}$ as before, we can define a homomorphism $G_{i} \in \operatorname{Hom}_{R}\left(I_{2}, R / I_{2}\right)$ (for $0 \leq i \leq s$ ) which takes $\alpha_{i}^{\prime}$ to $\alpha_{k}$ and all the other canonical generators of $I_{2}$ to zero. It is easy to check that such $G_{i}$ s form a basis for $\operatorname{Ker} \psi$ and thus, the dimension of Ker $\psi$ is equal to $s+1$.

From the exact sequence

$$
\begin{aligned}
0 \rightarrow \operatorname{Ker}(\phi-\psi) & \rightarrow \operatorname{Hom}_{R}\left(I_{1}, R / I_{1}\right) \oplus \operatorname{Hom}_{R}\left(I_{2}, R / I_{2}\right) \\
\underset{\phi-\psi}{\longrightarrow} & \operatorname{Hom}_{R}\left(I_{2}, R / I_{1}\right) \rightarrow 0,
\end{aligned}
$$

we obtain

$\operatorname{dim}(\operatorname{Ker}(\phi-\psi))=2(n-1)+\operatorname{dim}(\operatorname{Im} \psi)+(s+1)-\operatorname{dim}\left(\operatorname{Hom}_{R}\left(I_{2}, R / I_{1}\right)\right)$

and from (2.6.1), (2.6.2), (2.6.3) and (2.6.4), we observe that

$$
\operatorname{dim}\left(\operatorname{Hom}_{R}\left(I_{2}, R / I_{1}\right)\right)-\operatorname{dim}(\operatorname{Im} \psi)=s-1 .
$$

The required result is now immediate from Equations (2.6.5) and (2.6.6).

Proposition 2.6.4. If the action of $\mathbb{C}^{*}$ on $R=\mathbb{C}[x, y]$ defined in (2.0.1) is such that $0<\mu_{1}<\mu_{2}$ and $\frac{\mu_{2}}{\mu_{1}} \gg 0$, the part of the tangent space $T^{\prime}$ to $Z_{n-1, n}\left(\mathbb{A}^{2}\right)$ at the fixed point $\left(I_{1}, I_{2}\right)$ where the weights of $\mathbb{C}^{*}$ are positive has dimension $n-d$ where $d=\min \left\{i: x^{i} \in I_{2}\right\}$.

Proof. With the homomorphisms $F_{i} \mathrm{~s}$ in $\operatorname{Hom}_{R}\left(I_{2}, R / I_{1}\right)$ defined as in the proof of Lemma 2.6.2 and the homomorphisms $G_{i}$ s in Ker $\psi$ defined as in the proof of Proposition 2.6.3, let $F$ denote the number of $F_{i}$ s having positive weight and let $G$ denote the number of $G_{i}$ s having positive weight. Then, from the exact sequence

$$
\begin{aligned}
0 \rightarrow \operatorname{Ker}(\phi-\psi) & \rightarrow \operatorname{Hom}_{R}\left(I_{1}, R / I_{1}\right) \oplus \operatorname{Hom}_{R}\left(I_{2}, R / I_{2}\right) \\
\underset{\phi-\psi}{\longrightarrow} & \operatorname{Hom}_{R}\left(I_{2}, R / I_{1}\right) \rightarrow 0
\end{aligned}
$$


and the decompositions (2.6.1), (2.6.2), (2.6.3) and (2.6.4), one has

$$
\operatorname{dim}\left(T^{\prime}\right)^{+}=\operatorname{dim}\left(\operatorname{Hom}_{R}\left(I_{1}, R / I_{1}\right)\right)^{+}+G-F .
$$

It is easy to check that if $k=0$, the dimension of $\left(\operatorname{Hom}_{R}\left(I_{1}, R / I_{1}\right)\right)^{+}$is $n-d$ and $G$ is equal to $F$ while if $k \geq 0$, the dimension of $\left(\operatorname{Hom}_{R}\left(I_{1}, R / I_{1}\right)\right)^{+}$is $n-d-1$ and $G$ is equal to $F+1$. In both cases, we obtain from Equation (2.6.7) that $\left(T^{\prime}\right)^{+}$has dimension $n-d$.

\subsection{The tangent spaces at the fixed points of $Z_{\mathbf{n}}\left(\mathbb{A}^{1}\right)$.}

For $1 \leq i \leq m$, let $I_{i}$ be the ideal of $R=\mathbb{C}[x]$ generated by $x^{n_{i}}$. Then, the $m$-tuple $\left(I_{1}, I_{2}, \ldots, I_{m}\right)$ is the unique fixed point of $Z_{\mathbf{n}}\left(\mathbb{A}^{1}\right)$ under the $\mathbb{C}^{*}$ action described in (2.0.1). Recall that the tangent space $T_{\mathbf{n}}$ to the space $Z_{\mathbf{n}}\left(\mathbb{A}^{1}\right)$ at the point $\left(I_{1}, I_{2}, \ldots, I_{m}\right)$ is canonically isomorphic to the subspace $\bigcap_{i<j} \operatorname{Ker}\left(\left(\phi_{i, j}-\psi_{i, j}\right) \circ p_{i, j}\right)$ of $\underset{k=1}{\bigoplus} \operatorname{Hom}_{R}\left(I_{k}, R / I_{k}\right)$.

Proposition 2.7.1. The tangent space $T_{\mathbf{n}}$ at the unique point of the space $Z_{\mathbf{n}}\left(\mathbb{A}^{1}\right)$ fixed by the $\mathbb{C}^{*}$-action has dimension $n_{m}$.

Proof. We use induction on $m$. The base step involves checking that the tangent space $\operatorname{Hom}_{R}\left(\left\langle x^{n}\right\rangle, R /\left\langle x^{n}\right\rangle\right)$ to the Hilbert scheme $\operatorname{Hilb}^{n} \mathbb{A}^{1}$ at the point $\left\langle x^{n}\right\rangle$ has dimension $n$. The inductive step involves checking that $\left(f_{1}, f_{2}, \ldots, f_{m}\right)$ is in $T_{\mathbf{n}}$ if and only if $\left(f_{1}, f_{2}, \ldots, f_{m-1}\right)$ is in $T_{n_{1}, n_{2}, \ldots, n_{m-1}}$ and $f_{m}\left(x^{n_{m}}\right)$ is equal to

$$
a_{0} x^{n_{m-1}}+a_{1} x^{n_{m-1}+1}+\cdots+a_{n_{m}-n_{m-1}-1} x^{n_{m}-1}+x^{n_{m}-n_{m-1}} \zeta,
$$

where $\zeta$ satisfies $\bar{\zeta}=f_{m-1}\left(x^{n_{m-1}}\right)$ in $R / I_{m-1}$ and the $a_{i}$ 's are complex numbers.

Similarly, we can verify that:

Proposition 2.7.2. If the action of $\mathbb{C}^{*}$ on $R=\mathbb{C}[x]$ defined in (2.0.1) is such that $\mu>0$, then $\left(T_{\mathbf{n}}\right)^{+}=0$.

\section{Chapter III. Cellular decompositions for various nested Hilbert schemes.}

In [E-S], Ellingsrud and Strømme describe cellular decompositions for the spaces Hilb ${ }^{n} \mathbb{P}^{2}$, Hilb $^{n} \mathbb{A}^{2}$ and Hilb ${ }^{n}\left(\mathbb{A}^{2}, 0\right)$ by applying the results of Bialynicki-Birula [B1, B2] to a torus action to the natural action of a maximal torus of $S L(3)$ on Hilb ${ }^{n} \mathbb{P}^{2}$. 
We wish to look similarly at cellular decompositions defined by a torus action to the natural action of a maximal torus of $S L(r+1)$ on the schemes $Z_{\mathbf{n}}\left(\mathbb{P}^{r}\right), Z_{\mathbf{n}}\left(\mathbb{A}^{r}\right)$ and $\mathcal{Z}_{\mathbf{n}}\left(\mathbb{A}^{r}, 0\right)$ in all the cases where $Z_{\mathbf{n}}\left(\mathbb{P}^{r}\right)$ is smooth.

More precisely, we look at the torus actions on the schemes

$$
\operatorname{Hilb}^{2} \mathbb{P}^{r}, \operatorname{Hilb}^{3} \mathbb{P}^{r}, Z_{1,2}\left(\mathbb{P}^{r}\right), Z_{2,3}\left(\mathbb{P}^{r}\right), \operatorname{Hilb}^{n} \mathbb{P}^{2}, Z_{n-1, n}\left(\mathbb{P}^{2}\right) \text { and } Z_{\mathbf{n}}\left(\mathbb{P}^{1}\right)
$$

for arbitrary $r$ and $n$. Whereas Ellingsrud and Strømme quote the smoothness of $\operatorname{Hilb}^{n}\left(\mathbb{P}^{2}\right)$, which is proved by Fogarty in $[\mathbf{F o}]$, we start by proving that each scheme $Z_{\mathbf{n}}\left(\mathbb{P}^{r}\right)$ in the list (3.0.1) is smooth by showing that the tangent spaces to all of its fixed points under the torus action have the same dimension $r n_{m}$.

By the theorem of Chapter I, there are no smooth nested Hilbert schemes on projective space except for $\mathrm{Hilb}^{1} \mathbb{P}^{r}$ and those listed in (3.0.1). Given an arbitrary smooth $r$-dimensional quasiprojective variety $X$, we know that any point on $Z_{\mathbf{n}}(X)$ has an analytic neighbourhood which is isomorphic to a neighbourhood of some point on $Z_{\mathbf{n}}\left(\mathbb{P}^{r}\right)$. Hence, in fact, we have:

Theorem 3.0.1. If $X$ is a smooth $r$-dimensional quasiprojective variety, then the nested Hilbert scheme $Z_{\mathbf{n}}(X)$ is smooth if and only if it satisfies (0.2.1).

Following Ellingsrud and Strømme, the cellular decompositions which we obtain for the smooth nested Hilbert schemes on affine and projective space as well as for the corresponding nested Hilbert schemes listed in (0.2.2) are used to study the Borel-Moore homology groups and the Chow groups of these spaces.

Before we begin, let us recall a result which is fundamental in this chapter. Following Fulton ([Fu], Example 1.9.1), we say a scheme $X$ has a cellular decomposition if there is a filtration $X=X_{n} \supset X_{n-1} \supset \ldots \supset X_{0} \supset X_{-1}=$ $\emptyset$ by closed subschemes with each $X_{i} \backslash X_{i-1}$ a disjoint union of schemes $U_{i, j}$ isomorphic to affine spaces $\mathbb{A}^{n_{i, j}}$. The $U_{i, j}$ s are called the cells of the decomposition.

Theorem 3.0.2 (Bialynicki-Birula, $[\mathbf{B} 1, \mathbf{B} 2])$. Let $X$ be a smooth projective variety with an action of $\mathbb{C}^{*}$. Suppose that the set of fixed points $\left\{x_{1}, \ldots, x_{p}\right\}$ is finite and let $X_{i}=\left\{x \in X: \lim _{t \rightarrow 0} t x=x_{i}\right\}$. Then,

(1) $X$ has a cellular decomposition with cells $X_{i}$,

(2) $T_{x_{i}} X_{i}=\left(T_{x_{i}} X\right)^{+}$.

Remark. Let $X$ be a smooth projective variety with an action of a torus $G$, and suppose that the set of fixed points is finite. Then, $G$ induces a linear 
action on the tangent space to each fixed point. Any linear representation of $G$ splits up in a direct sum of one-dimensional representations corresponding to characters of $G$. Let $\left\{\chi_{1}, \chi_{2}, \ldots, \chi_{q}\right\}$ be the set of all characters of $G$ occuring in the tangent spaces to the fixed points and let $\tau: \mathbb{C}^{*} \rightarrow G$ be a one-parameter subgroup. Then, the set of fixed points of the induced action of $\mathbb{C}^{*}$ is the same as the set of $G$-fixed points if and only if $\chi_{j} \circ \tau$ are non-trivial characters of $\mathbb{C}^{*}$ for all $j=1,2, \ldots, q$. In particular, for "general" $\tau$ chosen outside $q$ given hyperplanes in the lattice of one-parameter subgroups, we get an action of $\mathbb{C}^{*}$ on $X$ with the same set of fixed points as the action of $G$ on $X$.

\subsection{An action of an $r$-dimensional torus on $Z_{\mathbf{n}}\left(\mathbb{P}^{r}\right)$.}

From now on, we fix a system of coordinates $Y_{0}, Y_{1}, \ldots, Y_{r}$ of $\mathbb{P}^{r}$. Let $G \subset S L(r+1, \mathbb{C})$ be the maximal torus consisting of all diagonal matrices. We denote by $\lambda_{0}, \lambda_{1}, \ldots, \lambda_{r}$ the complex characters of $G$ such that $g=$ $\operatorname{diag}\left(\lambda_{0}(g), \lambda_{1}(g), \ldots, \lambda_{r}(g)\right)$ for all $g \in G$. Then, $G$ acts on $\mathbb{P}^{r}$ via $g . Y_{i}=$ $\lambda_{i}(g) Y_{i}$ and on points $\left[a_{0}: a_{1}: \ldots: a_{r}\right]$, this action is given by $g\left[a_{0}: a_{1}: \ldots\right.$ : $\left.a_{r}\right]=\left[\lambda_{0}(g)^{-1} a_{0}: \lambda_{1}(g)^{-1} a_{1}: \ldots: \lambda_{r}(g)^{-1} a_{r}\right]$.

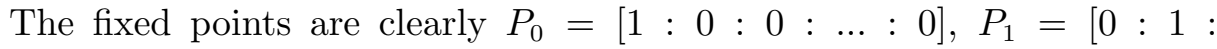
$0: \ldots: 0], \ldots, P_{r}=[0: 0: \ldots: 0: 1]$. For $0 \leq i \leq r$, let $F_{i}=$ $\left\langle P_{0}, P_{1}, \ldots, P_{i}\right\rangle \backslash\left\langle P_{0}, P_{1}, \ldots, P_{i-1}\right\rangle$. Here, $\left\langle P_{0}, P_{1}, \ldots, P_{i}\right\rangle$ denotes the linear span of the points $P_{0}, P_{1}, \ldots, P_{i}$. Then, $F_{i} \sim \mathbb{A}^{i}$ and the $F_{i}$ s define a cellular decomposition of $\mathbb{P}^{r}$.

The one-parameter subgroups $\tau: \mathbb{C}^{*} \rightarrow G$ inducing this cellular decomposition are those of the type $\tau(t)=\operatorname{diag}\left(t^{w_{0}}, t^{w_{1}}, \ldots, t^{w_{r}}\right)$ where $w_{0}<w_{1}<$ $\cdots<w_{r}$ and $w_{0}+w_{1}+w_{2}+\cdots+w_{r}=0$.

The action of $G$ on $\mathbb{P}^{r}$ induces naturally an action of $G$ on $Z_{\mathbf{n}}\left(\mathbb{P}^{r}\right)$.

For any $\left(Z_{1}, Z_{2}, \ldots, Z_{m}\right)$ in $Z_{\mathbf{n}}\left(\mathbb{P}^{r}\right)$, we can write each $Z_{i}$ uniquely as a disjoint union

$$
Z_{i}=Z_{i, 0} \cup Z_{i, 1} \cup \ldots \cup Z_{i, r},
$$

where $Z_{i, j}$ is a subscheme of $\mathbb{P}^{r}$ supported in $F_{j}$. Given any $m \times(r+1)$ matrix $\left(n_{i, j}\right)_{\substack{1 \leq i \leq m \\ 0 \leq j \leq r}}$ of non-negative integers with $\sum_{j=0}^{r} n_{i, j}=n_{i}$ for $1 \leq$ $i \leq m$, we define $S\left(n_{i, j}\right)$ to be the subset of $Z_{\mathbf{n}}\left(\mathbb{P}^{r}\right)$ parametrizing $m$-tuples $\left(Z_{1}, Z_{2}, \ldots, Z_{m}\right)$ of subschemes of $\mathbb{P}^{r}$ such that length $\left(\mathcal{O}_{Z_{i, j}}\right)=n_{i, j}$. Clearly, the nested Hilbert scheme $Z_{\mathbf{n}}\left(\mathbb{P}^{r}\right)$ is a disjoint union of such $S\left(n_{i, j}\right)$ s.

If the point $\left(Z_{1}, Z_{2}, \ldots, Z_{m}\right)$ of $Z_{\mathbf{n}}\left(\mathbb{P}^{r}\right)$ corresponds to a fixed point of this action, then each $Z_{i}$ is a subscheme of $\mathbb{P}^{r}$ whose support is contained in the set of fixed points $\left\{P_{0}, P_{1}, \ldots, P_{r}\right\}$ of $G$. Hence, if $Z_{i}$ is written as a union of subschemes as in Equation (3.1.1), the subscheme $Z_{i, j}$ is supported in $P_{j}$. 
For each $j,\left(Z_{1, j}, Z_{2, j}, \ldots, Z_{m, j}\right)$ is an $m$-tuple of subschemes of $\mathbb{P}^{r}$ supported at the point $P_{j}$ and it corresponds to a $G$-fixed point of $Z_{n_{1, j}, n_{2, j}, \ldots, n_{m, j}}\left(\mathbb{P}^{r}\right)$, where $n_{i, j}=$ length $\left(\mathcal{O}_{Z_{i, j}}\right)$.

Lemma 3.1.1. The action of $G$ on $Z_{\mathbf{n}}\left(\mathbb{P}^{r}\right)$ has only finitely many fixed points.

Proof. A point $\left(Z_{1}, Z_{2}, \ldots, Z_{m}\right)$ of $Z_{\mathbf{n}}\left(\mathbb{P}^{r}\right)$ is fixed if and only if each of the $Z_{i}$ s is a fixed point in Hilb ${ }^{n_{i}} \mathbb{P}^{r}$. A point of Hilb ${ }^{n_{i}} \mathbb{P}^{r}$ is a fixed point if and only if the corresponding ideal $I$ in $\mathbb{C}\left[Y_{0}, Y_{1}, \ldots, Y_{r}\right]$ is invariant under $G$, which is the case if and only if $I$ is generated by monomials. These ideals obviously form a finite family.

3.2. The smoothness of the nested Hilbert schemes listed in (3.0.1). First, we prove a lemma which reduces the problem of showing that a given nested Hilbert scheme $Z_{\mathbf{n}}\left(\mathbb{P}^{r}\right)$ is smooth to counting the dimensions of the tangent spaces at its $G$-fixed points. As remarked earlier, these are the same as the fixed points of $Z_{\mathbf{n}}\left(\mathbb{P}^{r}\right)$ under the action of $\mathbb{C}^{*}$ induced by a sufficiently general one-parameter subgroup $\tau: \mathbb{C}^{*} \rightarrow G$.

Lemma 3.2.1. If there is a $\mathbb{C}^{*}$-action on $Z_{\mathbf{n}}\left(\mathbb{P}^{r}\right)$ with isolated fixed points and the tangent space at each fixed point has dimension $r n_{m}$, then $Z_{\mathbf{n}}\left(\mathbb{P}^{r}\right)$ is a smooth irreducible variety of dimension $\mathrm{rn}_{m}$.

Proof. The dimension of the tangent space at any point of $Z_{\mathbf{n}}\left(\mathbb{P}^{r}\right)$ is less than or equal to $r n_{m}$ because if there is a point $P$ at which the tangent space has dimension greater than $r n_{m}, t . P$ must approach a $\mathbb{C}^{*}$-fixed point whose tangent space has dimension greater than $r n_{m}$ as $t \rightarrow 0$, contradicting the hypothesis of the lemma.

Let $\operatorname{Hilb}_{\neq}^{n} \mathbb{P}^{r}$ denote the open subset of $\operatorname{Hilb}^{n} \mathbb{P}^{r}$ parametrizing $n$ distinct points and let $p_{m}$ denote the projection from $Z_{\mathbf{n}}\left(\mathbb{P}^{r}\right)$ to Hilb ${ }^{n_{m}} \mathbb{P}^{r}$. Then, the closure $\bar{U}$ of $U=p_{m}{ }^{-1}\left(\right.$ Hilb $\left._{\neq}^{n_{m}} \mathbb{P}^{r}\right)$ is a component of $Z_{\mathbf{n}}\left(\mathbb{P}^{r}\right)$ of dimension $r n_{m}$. It is clear that the tangent space at any point on the component $\bar{U}$ has dimension $r n_{m}$.

The nested Hilbert scheme $Z_{\mathbf{n}}\left(\mathbb{P}^{r}\right)$ has no other component since if it had another component, this component would meet $\bar{U}$ by the connectedness of $Z_{\mathbf{n}}\left(\mathbb{P}^{r}\right)$ and the tangent space at any point where these components met would have dimension greater than $r n_{m}$.

Consider a $G$-fixed point $\left(Z_{1}, Z_{2}, \ldots, Z_{m}\right)$ on a nested Hilbert scheme $Z_{\mathbf{n}}\left(\mathbb{P}^{r}\right)$. With the notation of (3.1.1), let $T_{j}$ be the tangent space to 
$Z_{n_{1, j}, n_{2, j}, \ldots, n_{m, j}}\left(\mathbb{P}^{r}\right)$ at the $G$-fixed point corresponding to the $m$-tuple $\left(Z_{1, j}\right.$, $\left.Z_{2, j}, \ldots, Z_{m, j}\right)$ of subschemes of $\mathbb{P}^{r}$ concentrated at $P_{j}$. Then, the tangent space to $Z_{\mathbf{n}}\left(\mathbb{P}^{r}\right)$ at $\left(Z_{1}, Z_{2}, \ldots, Z_{m}\right)$ is isomorphic to the direct sum of the $T_{j} \mathrm{~s}$.

Let $\mathbf{s}=\left(s_{1}, s_{2}, \ldots, s_{k}\right)$ and $\mathbf{n}=\left(n_{1}, n_{2}, \ldots, n_{m}\right)$ be two increasing sequences of non-negative integers. We say that $\mathbf{s} \leq \mathbf{n}$ if $k \leq m, n_{1}-s_{1} \leq$ $n_{2}-s_{2} \leq \ldots \leq n_{k}-s_{k}$ and for any $1 \leq i \leq k, s_{i} \leq n_{i}$. To study the tangent spaces at the $G$-fixed points of $Z_{\mathbf{n}}\left(\mathbb{P}^{r}\right)$, we need only look at the nested Hilbert schemes $Z_{\mathbf{s}}\left(\mathbb{P}^{r}\right)$ for which $\mathbf{s} \leq \mathbf{n}$ and study the tangent spaces at their $G$-fixed points which correspond to $m$-tuples $\left(Z_{1}, Z_{2}, \ldots, Z_{m}\right)$ of subschemes of $\mathbb{P}^{r}$ concentrated at a single point of $\mathbb{P}^{r}$. For any such $m$ tuple $\left(Z_{1}, Z_{2}, \ldots, Z_{m}\right)$, the $Z_{i}$ s are contained in a $G$-invariant affine space $\mathbb{A}^{r}$. Hence, to show that the tangent spaces at all the $G$-fixed points of $Z_{\mathbf{n}}\left(\mathbb{P}^{r}\right)$ have dimension $r n_{m}$, we only have to show that whenever $\mathbf{s} \leq \mathbf{n}$, the tangent spaces at all the $G$-fixed points of $Z_{\mathbf{s}}\left(\mathbb{A}^{r}\right)$ have dimension $r s_{k}$. In fact, the $G$-fixed points of $Z_{\mathbf{s}}\left(\mathbb{A}^{r}\right)$ are the same as the points of $Z_{\mathbf{s}}\left(\mathbb{A}^{r}\right)$ fixed under the action of $\mathbb{C}^{*}$ induced by a sufficiently general one-parameter subgroup $\tau: \mathbb{C}^{*} \rightarrow G$ and such a $\mathbb{C}^{*}$-action on $Z_{\mathbf{s}}\left(\mathbb{A}^{r}\right)$ is described in the first paragraph of Chapter II. Recall that for a sufficiently general $\mathbb{C}^{*}$-action of this type,

(1) The tangent space to $\operatorname{Hilb}^{2} \mathbb{A}^{r}$ at any $\mathbb{C}^{*}$-fixed point has dimension $2 r$ [Prop. 2.1.1];

(2) The tangent space to $\operatorname{Hilb}^{3} \mathbb{A}^{r}$ at any $\mathbb{C}^{*}$-fixed point has dimension $3 r$ [Prop. 2.2.1 and Prop. 2.2.3];

(3) The tangent space to $Z_{1,2}\left(\mathbb{A}^{r}\right)$ at any $\mathbb{C}^{*}$-fixed point has dimension $2 r$ [Prop. 2.3.1];

(4) The tangent space to $Z_{2,3}\left(\mathbb{A}^{r}\right)$ at any $\mathbb{C}^{*}$-fixed point has dimension $3 r$ [Prop. 2.4.1 and Prop. 2.4.3];

(5) The tangent space to $\operatorname{Hilb}^{n} \mathbb{A}^{2}$ at any $\mathbb{C}^{*}$-fixed point has dimension $2 n$ [Prop. 2.5.4];

(6) The tangent space to $Z_{n-1, n}\left(\mathbb{A}^{2}\right)$ at any $\mathbb{C}^{*}$-fixed point has dimension $2 n$ [Prop. 2.6.3];

(7) The tangent space to $Z_{\mathbf{n}}\left(\mathbb{A}^{1}\right)$ at any $\mathbb{C}^{*}$-fixed point has dimension $n_{m}$ [Prop. 2.7.1].

It follows that:

(1) The tangent space to $\mathrm{Hilb}^{2} \mathbb{P}^{r}$ at any $G$-fixed point has dimension $2 r$;

(2) The tangent space to $\mathrm{Hilb}^{3} \mathbb{P}^{r}$ at any $G$-fixed point has dimension $3 r$;

(3) The tangent space to $Z_{1,2}\left(\mathbb{P}^{r}\right)$ at any $G$-fixed point has dimension $2 r$;

(4) The tangent space to $Z_{2,3}\left(\mathbb{P}^{r}\right)$ at any $G$-fixed point has dimension $3 r$;

(5) The tangent space to $\mathrm{Hilb}^{n} \mathbb{P}^{2}$ at any $G$-fixed point has dimension $2 n$;

(6) The tangent space to $Z_{n-1, n}\left(\mathbb{P}^{2}\right)$ at any $G$-fixed point has dimension $2 n$; 
(7) The tangent space to $Z_{\mathbf{n}}\left(\mathbb{P}^{1}\right)$ at any $G$-fixed point has dimension $n_{m}$. Hence, we deduce from Lemma 3.2.1 that:

Theorem 3.2.2. If $r$ and $n$ are arbitrary non-negative integers and $\mathbf{n}$ is an $m$-tuple of increasing non-negative integers, the schemes

$$
\operatorname{Hilb}^{2} \mathbb{P}^{r}, \operatorname{Hilb}^{3} \mathbb{P}^{r}, Z_{1,2}\left(\mathbb{P}^{r}\right), Z_{2,3}\left(\mathbb{P}^{r}\right), \operatorname{Hilb}^{n} \mathbb{P}^{2}, Z_{n-1, n}\left(\mathbb{P}^{2}\right) \text { and } Z_{\mathbf{n}}\left(\mathbb{P}^{1}\right)
$$

are smooth.

3.3. Cellular decompositions for the smooth nested Hilbert schemes on affine and projective space and for the corresponding punctual nested Hilbert schemes; the homology groups of these spaces.

For any complex variety $X$, let $H_{*}(X)$ be the Borel-Moore homology of $X$ (homology with locally finite supports). By the $i^{\text {th }}$ Betti number $b_{i}(X)$, we shall mean the rank of the finitely-generated abelian group $H_{i}(X)$ and by the Poincaré polynomial $P(X)$, we mean the polynomial $\sum_{k} b_{k}(X) z^{k}$. As usual, $A_{*}(X)$ is the Chow group of $X$ and $\mathrm{cl}: A_{*}(X) \rightarrow H_{*}(X)$ is the cycle map. If $X$ is compact, the Borel-Moore homology of $X$ agrees with the usual singular homology.

Proposition 3.3.1. Let $X$ be one of the smooth nested Hilbert schemes on affine or projective space or one of the corresponding punctual nested Hilbert schemes listed in (0.2.2). Then, the cycle map cl : $A_{*}(X) \rightarrow H_{*}(X)$ is an isomorphism and in particular, the odd homology vanishes. Furthermore, both groups are free abelian groups and the rank of $H_{2 k}(X)$ is equal to the number of $k$-cells in the cellular decomposition of $X$.

To prove Proposition 3.3.1, we need the following proposition from Chapter 19.1 of $[\mathbf{F u}]$.

Proposition 3.3.2. $\quad$ Let $X$ be a scheme with a cellular decomposition. Then, for $0 \leq i \leq \operatorname{dim} X$,

(i) $H_{2 i+1}(X)=0$,

(ii) $H_{2 i}(X)$ is a $\mathbb{Z}$-module freely generated by the classes of the closures of the $i$-dimensional cells,

(iii) The cycle map $\mathrm{cl}: A_{*}(X) \rightarrow H_{*}(X)$ is an isomorphism.

Proof of Proposition 3.3.1. Let $Z_{\mathbf{n}}\left(\mathbb{P}^{r}\right)$ be a nested Hilbert scheme in the list (3.0.1). Since $Z_{\mathbf{n}}\left(\mathbb{P}^{r}\right)$ is smooth and projective [Gr], we may apply Theorem 3.0.2 to the action of any sufficiently general one-parameter subgroup of $G$ 
on $Z_{\mathbf{n}}\left(\mathbb{P}^{r}\right)$ so that $Z_{\mathbf{n}}\left(\mathbb{P}^{r}\right)$ has a cellular decomposition and we can apply Proposition 3.3.2.

As described in Section 3.1, the nested Hilbert scheme $Z_{\mathbf{n}}\left(\mathbb{P}^{r}\right)$ is a disjoint union of subsets of the form $S\left(n_{i, j}\right)$. Let $\tau$ be any one-parameter subgroup of $G$ respecting the cellular decomposition $\left\{F_{0}, F_{1}, \ldots, F_{r}\right\}$ of $\mathbb{P}^{r}$. Then, as $t \rightarrow$ $0, \tau(t)\left(Z_{i, j}\right)$ approaches a subscheme supported in $P_{j}$. In other words, any point in $S\left(n_{i, j}\right)$ approaches a fixed point in $S\left(n_{i, j}\right)$. Since the cell of $Z_{\mathbf{n}}\left(\mathbb{P}^{r}\right)$ associated to the fixed point $P$ is exactly the set $\left\{x \in P: \lim _{t \rightarrow 0} t . x=P\right\}$, $S\left(n_{i, j}\right)$ is the union of the cells of $Z_{\mathbf{n}}\left(\mathbb{P}^{r}\right)$ associated to the fixed points in $S\left(n_{i, j}\right)$.

In particular, as $Z_{\mathbf{n}}\left(\mathbb{A}^{r}\right)$ is isomorphic to

$$
S\left(\begin{array}{ccccc}
0 & 0 & \ldots & 0 & n_{1} \\
0 & 0 & \ldots & 0 & n_{2} \\
\vdots & \vdots & \vdots & \vdots & \vdots \\
0 & 0 & \ldots & 0 & n_{m}
\end{array}\right),
$$

it has a cellular decomposition and Proposition 3.3.2 applies to it.

Similarly, since the punctual nested Hilbert scheme $\mathcal{Z}_{\mathbf{n}}\left(\mathbb{A}^{r}, 0\right)$ is isomorphic to the set

$$
S\left(\begin{array}{ccccc}
n_{1} & 0 & 0 & \ldots & 0 \\
n_{2} & 0 & 0 & \ldots & 0 \\
\vdots & \vdots & \vdots & \vdots & \vdots \\
n_{m} & 0 & 0 & \ldots & 0
\end{array}\right),
$$

it too has a cellular decomposition and Proposition 3.3.2 again applies.

Next, we calculate the number of cells of each dimension and the Betti numbers of the nested punctual Hilbert scheme listed in (0.2.2).

Theorem 3.3.3. If $r$ and $n$ are arbitrary non-negative integers and $\mathbf{n}$ is an $m$-tuple of increasing non-negative integers,

(1) $\sum_{i}\left(\right.$ No. of $i$-cells in $\left.\operatorname{Hilb}^{2}\left(\mathbb{A}^{r}, 0\right)\right) v^{i}=\sum_{i=1}^{r-1} v^{i}$,

(2) $\sum_{i}\left(\right.$ No. of $i$-cells in $\left.\operatorname{Hilb}^{3}\left(\mathbb{A}^{r}, 0\right)\right) v^{i}=\sum_{i=0}^{r-1} v^{2 i}+\sum_{0 \leq i<j \leq r-1} v^{i+j}=$ $\sum_{0 \leq i \leq j \leq r-1} v^{i+j}$,

(3) $\sum_{i}\left(\right.$ No. of $i$-cells in $\left.\mathcal{Z}_{2,3}\left(\mathbb{A}^{r}, 0\right)\right) v^{i}=\sum_{i=0}^{r-1} v^{2 i}+2 \sum_{0 \leq i<j \leq r-1} v^{i+j}=$ $\sum_{i=0}^{r-1} \sum_{j=0}^{r-1} v^{i+j}$

(4) $\sum_{k=0}^{\infty} \sum_{i}\left(\right.$ No. of $i$-cells in $\left.\operatorname{Hilb}^{k}\left(\mathbb{A}^{2}, 0\right)\right) v^{i} t^{k}=\prod_{k=1}^{\infty}\left(\frac{1}{1-t^{k} v^{k-1}}\right)$

$[\mathbf{E}-\mathrm{S}]$, 
(5) $\sum_{k=1}^{\infty} \sum_{i}\left(\right.$ No. of $i$-cells in $\left.\mathcal{Z}_{k-1, k}\left(\mathbb{A}^{2}, 0\right)\right) v^{i} t^{k}$

$=\left(\frac{t}{1-t v}\right)\left(\prod_{k=1}^{\infty}\left(\frac{1}{1-t^{k} v^{k-1}}\right)\right)$,

(6) $\quad \sum_{i}\left(\right.$ No. of $i$-cells in $\left.\mathcal{Z}_{\mathbf{n}}\left(\mathbb{A}^{1}, 0\right)\right) v^{i}=1$.

Corollary. If $r$ and $n$ are arbitrary non-negative integers and $\mathbf{n}$ is an $m$-tuple of increasing non-negative integers,

(1) $P\left(\operatorname{Hilb}^{2}\left(\mathbb{A}^{r}, 0\right)\right)=\sum_{i=0}^{r-1} z^{2 i}$,

(2) $P\left(\operatorname{Hilb}^{3}\left(\mathbb{A}^{r}, 0\right)\right)=\sum_{i=0}^{r-1} z^{4 i}+\sum_{0 \leq i<j \leq r-1} z^{2(i+j)}=\sum_{0 \leq i \leq j \leq r-1} z^{2(i+j)}$,

(3) $P\left(\mathcal{Z}_{2,3}\left(\mathbb{A}^{r}, 0\right)\right)=\sum_{i=0}^{r-1} z^{4 i}+2 \sum_{0 \leq i<j \leq r-1} z^{2(i+j)}=\sum_{i=0}^{r-1} \sum_{j=0}^{r-1} z^{2(i+j)}$,

(4) $\sum_{k=0}^{\infty} P\left(\operatorname{Hilb}^{k}\left(\mathbb{A}^{2}, 0\right)\right) t^{k}=\prod_{k=1}^{\infty}\left(\frac{1}{1-t^{k} z^{2(k-1)}}\right)$,

(5) $\sum_{k=1}^{\infty} P\left(\mathcal{Z}_{k-1, k}\left(\mathbb{A}^{2}, 0\right)\right) t^{k}=\left(\frac{t}{1-t z^{2}}\right)\left(\prod_{k=1}^{\infty}\left(\frac{1}{1-t^{k} z^{2(k-1)}}\right)\right)$,

(6) $P\left(\mathcal{Z}_{\mathbf{n}}\left(\mathbb{A}^{1}, 0\right)\right)=1$.

Proof of Theorem 3.3.3. All the subschemes of $\mathbb{P}^{r}$ corresponding to the points in

$$
S\left(\begin{array}{ccccc}
n_{1} & 0 & 0 & \ldots & 0 \\
n_{2} & 0 & 0 & \ldots & 0 \\
\vdots & \vdots & \vdots & \vdots & \vdots \\
n_{m} & 0 & 0 & \ldots & 0
\end{array}\right)
$$

are contained in the affine space $\mathbb{C}\left[Y_{1} / Y_{0}, Y_{2} / Y_{0}, \ldots, Y_{r} / Y_{0}\right]$. We put $x_{i}=$ $Y_{i} / Y_{0}$ for $1 \leq i \leq r$. Define a one-parameter subgroup $\tau: \mathbb{C}^{*} \rightarrow G$ by $\tau(t)=$ $\operatorname{diag}\left(t^{w_{0}}, t^{w_{1}}, \ldots, t^{w_{r}}\right)$, where $w_{0}<w_{1}<\cdots<w_{r}$ and $w_{0}+w_{1}+\cdots+w_{r}=0$. In fact, we may assume that $\frac{w_{j}-w_{0}}{w_{i}-w_{0}} \gg 0$ whenever $r \geq j>i \geq 1$. Pick a cell $U$ from the cellular decomposition of $Z_{\mathbf{n}}\left(\mathbb{P}^{r}\right)$ defined by $\tau$ contained in

$$
S\left(\begin{array}{ccccc}
n_{1} & 0 & 0 & \ldots & 0 \\
n_{2} & 0 & 0 & \ldots & 0 \\
\vdots & \vdots & \vdots & \vdots & \vdots \\
n_{m} & 0 & 0 & \ldots & 0
\end{array}\right) .
$$

We want to compute its dimension. The cell $U$ corresponds to a fixed point of $G$ on $Z_{\mathbf{n}}\left(\mathbb{P}^{r}\right)$ inside Spec $\mathbb{C}\left[Y_{1} / Y_{0}, Y_{2} / Y_{0}, \ldots, Y_{r} / Y_{0}\right]=\operatorname{Spec} \mathbb{C}\left[x_{1}, x_{2}, \ldots, x_{r}\right]$, hence to a nest of invariant ideals $I_{1} \supset I_{2} \supset \ldots \supset I_{m}$ in $\mathbb{C}\left[x_{1}, x_{2}, \ldots, x_{r}\right]$. According to Theorem 3.0.2, $\operatorname{dim} U=\operatorname{dim} T^{+}$, where $T$ is the tangent space to $Z_{\mathbf{n}}\left(\mathbb{P}^{r}\right)$ at this fixed point.

Thus, we are reduced to calculating the positive parts of the tangent spaces at the fixed points of $Z_{\mathbf{n}}\left(\mathbb{A}^{r}\right)$ under the action of $\mathbb{C}^{*}$ described in the first paragraph of Chapter II. The assumptions that $w_{0}<w_{1}<\cdots<w_{r}$ 
and $\frac{w_{j}-w_{0}}{w_{i}-w_{0}} \gg 0$ whenever $r \geq j>i \geq 1$ translate to the assumptions that $0<\mu_{1}<\mu_{2}<\cdots<\mu_{r}$ and $\frac{\mu_{j}}{\mu_{i}} \gg 0$ whenever $r \geq j>i \geq 1$.

Proof of (1). Recall from Section 2.1 that $\operatorname{Hilb}^{2}\left(\mathbb{A}^{r}\right)$ has $r \mathbb{C}^{*}$-fixed points $I_{1}, I_{2}, \ldots, I_{r}$. By Proposition 2.1.2, the positive part of the tangent space to $\operatorname{Hilb}^{2}\left(\mathbb{A}^{r}\right)$ at $I_{i}$ has dimension $(i-1)$.

Proof of (2). Recall from Section 2.2 that the $\mathbb{C}^{*}$-fixed points of $\operatorname{Hilb}^{3}\left(\mathbb{A}^{r}\right)$ are $\tilde{I}_{i}(1 \leq i \leq r)$ and $\hat{I}_{i, j}(1 \leq i<j \leq r)$. By Proposition 2.2.2, the positive part of the tangent space to $\operatorname{Hilb}^{3}\left(\mathbb{A}^{r}\right)$ at $\tilde{I}_{i}$ has dimension $2(i-1)$ and by Proposition 2.2.4, the positive part of the tangent space to $\operatorname{Hilb}^{3}\left(\mathbb{A}^{r}\right)$ at $\hat{I}_{i, j}$ has dimension $(i+j-2)$.

Proof of (3). Recall from Section 2.4 that the $\mathbb{C}^{*}$-fixed points of $Z_{2,3}\left(\mathbb{A}^{r}\right)$ are $\left(I_{i}, \tilde{I}_{i}\right)(1 \leq i \leq r),\left(I_{i}, \hat{I}_{i, j}\right)(1 \leq i<j \leq r)$ and $\left(I_{j}, \hat{I}_{i, j}\right)(1 \leq i<j \leq r)$. By Proposition 2.4.2, the positive part of the tangent space to $Z_{2,3}\left(\mathbb{A}^{r}\right)$ at $\left(I_{i}, \tilde{I}_{i}\right)$ has dimension $2(i-1)$ and by Proposition 2.4 .4 , the positive part of the tangent space to $Z_{2,3}\left(\mathbb{A}^{r}\right)$ at $\left(I_{i}, \hat{I}_{i, j}\right)$ or $\left(I_{j}, \hat{I}_{i, j}\right)$ has dimension $(i+j-2)$.

Proof of (6). By Proposition 2.7.2, the positive part of the tangent space to $Z_{\mathbf{n}}\left(\mathbb{A}^{1}\right)$ at the unique $\mathbb{C}^{*}$-fixed point has dimension 0 .

Proof of (4). Recall from Section 2.5 that the $\mathbb{C}^{*}$-fixed points of $\operatorname{Hilb}^{k}\left(\mathbb{A}^{2}\right)$ correspond to the partitions of $k$. By Proposition 2.5.5, the positive part of the tangent space to $\operatorname{Hilb}^{k}\left(\mathbb{A}^{2}\right)$ at a $\mathbb{C}^{*}$-fixed point has dimension $(k-d)$ where $d$ is the largest part of the corresponding partition. Let $p(k, l)$ denote the number of partitions of $k$ whose largest part is $l$. Then,

$$
\begin{aligned}
\sum_{k=0}^{\infty} \sum_{i=0}^{\infty}\left(\text { No. of } i \text {-cells in } \operatorname{Hilb}^{k}\left(\mathbb{A}^{2}, 0\right)\right) v^{i} t^{k} & =\sum_{k=0}^{\infty} \sum_{i=0}^{\infty} p(k, k-i) t^{k} v^{i} \\
& =\prod_{k=1}^{\infty}\left(\frac{1}{1-t^{k} v^{k-1}}\right) .
\end{aligned}
$$

Proof of (5). Recall from Section 2.6 that the $\mathbb{C}^{*}$-fixed points of the space $Z_{k-1, k}\left(\mathbb{A}^{2}\right)$ correspond to the partitions of $k-1$ "sitting inside" partitions of $k$. By Proposition 2.6.4, the positive part of the tangent space to $Z_{k-1, k}\left(\mathbb{A}^{2}\right)$ at a $\mathbb{C}^{*}$-fixed point has dimension $k-d$, where $d$ is the largest part of the partition of $k$.

A partition of $k$ consisting of $s_{i}$ copies of $i$ is said to be a partition with $m$ steps if the set $\left\{i \in \mathbb{N}^{+}: s_{i} \neq 0\right\}$ has $m$ members. Clearly, there are exactly $m$ partitions of $k-1$ which are subpartitions of any given partition of $k$ with $m$ steps. Hence, if $A_{m, l}^{k}$ denotes the number of partitions of $k$ with $m$ steps whose largest part is equal to $k-l$, the cellular decomposition of $\mathcal{Z}_{k-1, k}\left(\mathbb{A}^{2}, 0\right)$ studied in this section has exactly $\left(\sum_{m} m A_{m, l}^{k}\right) l$-cells. 
From the discussion above, we need only show that

$$
\left(\frac{t}{1-t v}\right)\left(\prod_{k=1}^{\infty} \frac{1}{1-t^{k} v^{k-1}}\right)=\sum_{k, l}\left(\sum_{m} m A_{m, l}^{k}\right) v^{l} t^{k} .
$$

A sequence of natural numbers $\mathbf{s}=\left(s_{0}, s_{1}, s_{2}, \ldots\right)$ is said to satisfy condition $(*)_{m, l}^{k}$ if exactly $m$ of its terms are non-zero, $\sum_{i=0}^{\infty} s_{i}=k-l$ and $\sum_{i=1}^{\infty} i s_{i}=l$. We can associate to any such $\mathbf{s}$ a partition of $k$ with $\mathrm{m}$ steps whose largest part has size $k-l$ by adding a part of size $k-l$ to the conjugate of the partition of $l$ consisting of $s_{i}$ copies of $i$, as illustrated in the figure below.

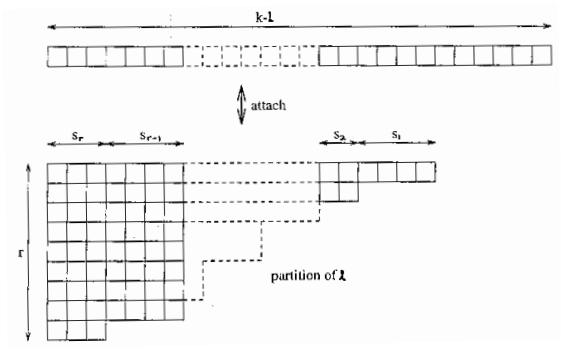

Figure 7.

In fact, any partition of $k$ whose largest part has size $k-l$ arises in this way so that there are exactly $A_{m, l}^{k}$ sequences s satisfying condition $(*)_{m, l}^{k}$. If

$$
p(u, t, x, y)=\prod_{k=0}^{\infty}\left(u\left(\frac{1}{1-t^{k+1}(x y)^{k}}-1\right)+1\right),
$$

it is not hard to check that the coefficient of $u^{m} t^{k}(x y)^{l}$ in $p(u, t, x, y)$ is the number of ss satisfying condition $(*)_{m, l}^{k}$ and hence,

$$
p(u, t, x, y)=\sum_{m, k, l} A_{m, l}^{k} u^{m}(x y)^{l} t^{k} .
$$

Differentiating both sides with respect to $u$ and setting $u=1$, we obtain Equation (3.3.1) as required.

\subsection{Cellular decompositions for the Hilbert function strata of various punctual Hilbert schemes.}

In [Gö1] and [Gö2], cellular decompositions are obtained for the Hilbert function strata of $\operatorname{Hilb}^{n}\left(\mathbb{A}^{2}, 0\right)$. This section contains straightforward modifications of Göttsche's arguments to obtain cellular decompositions for the "Hilbert function strata" of the other punctual Hilbert schemes which we have been studying. Let us first review some of the notation and results established in [Gö1] and [Gö2]. 


\section{4.a. Background and some small cases.}

Let $A=\mathbb{C}\left[\left[x_{1}, x_{2}, \ldots, x_{r}\right]\right]$ be the ring of formal power series in $r$ variables and let $\mathcal{M}=\left\langle x_{1}, x_{2}, \ldots, x_{r}\right\rangle$ be the maximal ideal of $A$.

Definition. Let $I \subset A$ be an ideal of colength $n$. The Hilbert function of $I$ is the sequence $T(I)=\left(t_{i}(I)\right)_{i \geq 0}$ of non-negative integers given by $t_{i}(I)=\operatorname{dim}_{k}\left(\mathcal{M}^{i} /\left(\left(I \cap \mathcal{M}^{i}\right)+\mathcal{M}^{i+1}\right)\right)$. If $T=\left(t_{i}\right)_{i \geq 0}$ is a sequence of non-negative integers, of which only finitely many do not vanish, we put $|T|=\sum_{i} t_{i}$. The initial degree $d$ of $T$ is the smallest $i$ such that $t_{i}<\left(\begin{array}{c}r+i-1 \\ i\end{array}\right)$.

Let $A_{i}=\mathcal{M}^{i} / \mathcal{M}^{i+1}$ and $I_{i}=\left(\mathcal{M}^{i} \cap I\right) /\left(\mathcal{M}^{i+1} \cap I\right)$. Then $A_{i}$ is the space of forms of degree $i$ in $A$ and $I_{i}$ the space of initial forms of $I$ (i.e. the forms of minimal degree among elements of $I$ ) of degree $i$, and we have $t_{i}(I)=\operatorname{dim}_{k}\left(A_{i} / I_{i}\right)$.

The Hilbert function gives us a stratification of $\operatorname{Hilb}^{n}(A)_{\text {red }} \sim \operatorname{Hilb}^{n}\left(\mathbb{A}^{r}, 0\right)$.

Definition. Let $T=\left(t_{i}\right)_{i \geq 0}$ be a sequence of non-negative integers with $|T|=n$. Let $M_{T} \subset \operatorname{Hilb}^{n}(A)_{\text {red }}$ be the locally closed subscheme (with the reduced induced structure) parametrizing ideals $I \subset A$ with Hilbert function $T$. Let $L_{T} \subset M_{T}$ be the closed subscheme (with the reduced induced structure) parametrizing homogeneous ideals $I \subset A$ with Hilbert function $T$. Let $\rho_{T}: M_{T} \rightarrow L_{T}$ be the morphism which maps an ideal $I$ to the associated homogeneous ideal (i.e. the ideal generated by the initial forms of elements of $I$ ). The embedding $L_{T} \subset M_{T}$ is a natural section of $\rho_{T}$.

If $\mathbf{T}=\left(T_{1}, T_{2}, \ldots, T_{m}\right)$ is an $m$-tuple of sequences of non-negative integers satisfying $\left|T_{i}\right|=n_{i}$, we may define

$$
\begin{aligned}
M_{\mathbf{T}} & :=\mathcal{Z}_{\mathbf{n}}\left(\mathbb{A}^{r}, 0\right) \cap\left(M_{T_{1}} \times M_{T_{2}} \times \cdots \times M_{T_{m}}\right) \quad \text { and } \\
L_{\mathbf{T}} & :=\mathcal{Z}_{\mathbf{n}}\left(\mathbb{A}^{r}, 0\right) \cap\left(L_{T_{1}} \times L_{T_{2}} \times \cdots \times L_{T_{m}}\right) .
\end{aligned}
$$

Again, there is a morphism $\rho_{\mathbf{T}}: M_{\mathbf{T}} \rightarrow L_{\mathbf{T}}$ for which the embedding $L_{\mathbf{T}} \subset$ $M_{\mathbf{T}}$ is a natural section. The $M_{\mathbf{T}} \mathrm{s}$ which are non-empty stratify $\mathcal{Z}_{\mathbf{n}}\left(\mathbb{A}^{r}, 0\right)$ and are called its Hilbert function strata.

\section{Examples.}

(1) $\mathcal{Z}_{\mathbf{n}}\left(\mathbb{A}^{1}, 0\right)=M_{(1)}=L_{(1)}=$ point.

(2) If $r \geq 1, \operatorname{Hilb}^{2}\left(\mathbb{A}^{r}, 0\right)=M_{(1,1)}=L_{(1,1)} \sim \mathbb{P}\left(\left(\mathcal{M} / \mathcal{M}^{2}\right)^{*}\right)$.

(3) If $r \geq 2, \operatorname{Hilb}^{3}\left(\mathbb{A}^{r}, 0\right)=M_{(1,2)} \cup M_{(1,1,1)}$. Here, $M_{(1,2)}=L_{(1,2)}$ is isomorphic to the Grassmannian $\operatorname{Grass}\left(2, \mathcal{M} / \mathcal{M}^{2}\right)$ parametrizing codimension 2 vector subspaces of $\mathcal{M} / \mathcal{M}^{2}$, while $M_{(1,1,1)}$ is a locally trivial $\mathbb{A}^{r-1}$-bundle over $L_{(1,1,1)} \sim \mathbb{P}\left(\left(\mathcal{M} / \mathcal{M}^{2}\right)^{*}\right)$.

(4) If $r \geq 2, \mathcal{Z}_{2,3}\left(\mathbb{A}^{r}, 0\right)=M_{(1,1),(1,2)} \cup M_{(1,1),(1,1,1)}$. Here, $M_{(1,1),(1,2)}=$ $L_{(1,1),(1,2)}$ is the flag variety in $\operatorname{Grass}\left(1, \mathcal{M} / \mathcal{M}^{2}\right) \times \operatorname{Grass}\left(2, \mathcal{M} / \mathcal{M}^{2}\right)$, 
while $M_{(1,1),(1,1,1)}$ is a locally trivial $\mathbb{A}^{r-1}$-bundle over $L_{(1,1),(1,1,1)} \sim$ $\mathbb{P}\left(\left(\mathcal{M} / \mathcal{M}^{2}\right)^{*}\right)$.

Suppose that $Z_{\mathbf{n}}\left(\mathbb{P}^{r}\right)$ is smooth. Then a general one-parameter subgroup $\tau: \mathbb{C}^{*} \rightarrow G ; t \mapsto \operatorname{diag}\left(t^{w_{0}}, t^{w_{1}}, \ldots, t^{w_{r}}\right)$ for which $w_{0}<w_{1}<\cdots<w_{r}$ and $w_{0}+w_{1}+\cdots+w_{r}=0$ induces a cellular decomposition of $Z_{\mathbf{n}}\left(\mathbb{P}^{r}\right)$ where the closed subset

$$
\mathcal{Z}_{\mathbf{n}}\left(\mathbb{A}^{r}, 0\right)=\left\{\left(Z_{1}, Z_{2}, \ldots, Z_{m}\right) \in Z_{\mathbf{n}}\left(\mathbb{P}^{r}\right): Z_{i} \text { is supported at the point } P_{0}\right\}
$$

is a union of cells. The next lemma asserts that if, in addition, the oneparameter subgroup $\tau$ satisfies $n_{m}\left(w_{i}-w_{0}\right)>\left(n_{m}-1\right)\left(w_{j}-w_{0}\right)$ whenever $r \geq j>i \geq 1$, then each of the Hilbert function strata of $\mathcal{Z}_{\mathbf{n}}\left(\mathbb{A}^{r}, 0\right)$ is itself a union of cells. An example of such a one-parameter subgroup can be constructed by letting $w_{0}=-r n_{m}-\frac{1}{2}(r-1) r$ and by letting $w_{j}=n_{m}+j-1$ when $1 \leq j \leq r$.

Lemma 3.4.1. Let $\mathcal{Z}_{\mathbf{n}}\left(\mathbb{A}^{r}, 0\right)$ be one of the punctual nested Hilbert schemes listed in (0.2.2). Let $\mathbb{C}^{*} \stackrel{\tau}{\rightarrow} G ; t \mapsto \operatorname{diag}\left(t^{w_{0}}, t^{w_{1}}, \ldots, t^{w_{r}}\right)$ be a general oneparameter subgroup of $G$ satisfying $w_{0}<w_{1}<\cdots<w_{r}, w_{0}+w_{1}+\cdots+w_{r}=0$ and $n_{m}\left(w_{i}-w_{0}\right)>\left(n_{m}-1\right)\left(w_{j}-w_{0}\right)$ whenever $r \geq j>i \geq 1$. Let $\mathbf{T}=\left(T_{1}, T_{2}, \ldots, T_{m}\right)$ be an $m$-tuple of sequences of non-negative integers satisfying $\left|T_{i}\right|=n_{i}$. Then,

(1) $M_{\mathbf{T}}$ is a union of cells of the cellular decomposition of $\mathcal{Z}_{\mathbf{n}}\left(\mathbb{A}^{r}, 0\right)$ induced by $\tau$,

(2) $\quad \rho_{\mathbf{T}}: M_{\mathbf{T}} \rightarrow L_{\mathbf{T}}$ is equivariant for the action of $\mathbb{C}^{*}$.

Proof. The proof is a straightforward modification of Göttsche's proof of parts (a) and (b) of [Gö1, Lemma 2.3].

Moreover, if $\mathcal{Z}_{\mathbf{n}}\left(\mathbb{A}^{r}, 0\right)$ is one of the punctual nested Hilbert schemes

$$
\operatorname{Hilb}^{2}\left(\mathbb{A}^{r}, 0\right), \operatorname{Hilb}^{3}\left(\mathbb{A}^{r}, 0\right), \mathcal{Z}_{2,3}\left(\mathbb{A}^{r}, 0\right) \text { or } \mathcal{Z}_{\mathbf{n}}\left(\mathbb{A}^{1}, 0\right)
$$

then we have already seen that any $L_{\mathbf{T}}$ is itself a smooth projective variety. It is clear that if $\left(I_{1}, I_{2}, \ldots, I_{m}\right)$ is in $L_{\mathbf{T}}$, then for any $t \in \mathbb{C}^{*}$, $\left(\tau(t) I_{1}, \tau(t) I_{2}, \ldots, \tau(t) I_{m}\right)$ will also be in $L_{\mathbf{T}}$. So, $\mathbb{C}^{*}$ acts on $L_{\mathbf{T}}$ and we can apply Theorem 3.0.2 to this action, yielding a cellular decomposition for $L_{\mathbf{T}}$. Since the action of $\mathbb{C}^{*}$ on $L_{\mathbf{T}}$ is the restriction of the action of $\mathbb{C}^{*}$ on $M_{\mathbf{T}}$, the cells of $L_{\mathbf{T}}$ are the intersection of the cells of $M_{\mathbf{T}}$ with $L_{\mathbf{T}}$.

We wish to calculate the number of $i$-cells in each of these $M_{\mathbf{T}} \mathrm{S}$ and $L_{\mathbf{T}} \mathrm{S}$. Since $\mathcal{Z}_{\mathbf{n}}\left(\mathbb{A}^{1}, 0\right)=M_{(1)}=L_{(1)}=$ point and $\operatorname{Hilb}^{2}\left(\mathbb{A}^{r}, 0\right)=M_{(1,1)}=$ $L_{(1,1)}$, there is no work to be done in these cases. For the spaces $\operatorname{Hilb}^{3}\left(\mathbb{A}^{r}, 0\right)$ and $\mathcal{Z}_{2,3}\left(\mathbb{A}^{r}, 0\right)$, it is not hard to check that, when $r \geq 2$, 
Proposition 3.4.2. (1) The punctual Hilbert scheme $\operatorname{Hilb}^{3}\left(\mathbb{A}^{r}, 0\right)$ is a disjoint union of the Hilbert function strata $M_{(1,1,1)}$ and $M_{(1,2)}$. If $\tau: \mathbb{C}^{*} \rightarrow G$ is a one-parameter subgroup of $G$ satisfying the hypothesis of Lemma 3.4.1, it induces cellular decompositions of $M_{(1,1,1)}, M_{(1,2)}, L_{(1,1,1)}$ and $L_{(1,2)}$ described by
(a) $\sum_{i}\left(\right.$ No. of $i$-cells in $\left.M_{(1,1,1)}\right) v^{i}=v^{r-1}\left(\sum_{i=0}^{r-1} v^{i}\right)$,
(b) $\sum_{i}\left(\right.$ No. of $i$-cells in $\left.M_{(1,2)}\right) v^{i}=\sum_{0 \leq i<j \leq r-1} v^{i+j-1}$,
(c) $\sum_{i}\left(\right.$ No. of $i$-cells in $\left.L_{(1,1,1)}\right) v^{i}=\sum_{i=0}^{r-1} v^{i} \quad$ and
(d) $\quad \sum_{i}\left(\right.$ No. of $i$-cells in $\left.L_{(1,2)}\right) v^{i}=\sum_{0 \leq i<j \leq r-1} v^{i+j-1}$.

(2) The punctual nested Hilbert scheme $\mathcal{Z}_{2,3}\left(\mathbb{A}^{r}, 0\right)$ is a disjoint union of the Hilbert function strata $M_{(1,1),(1,1,1)}$ and $M_{(1,1),(1,2)}$. If $\tau: \mathbb{C}^{*} \rightarrow G$ is a oneparameter subgroup of $G$ satisfying the hypothesis of Lemma 3.4.1, it induces cellular decompositions of $M_{(1,1),(1,1,1)}, M_{(1,1),(1,2)}, L_{(1,1),(1,1,1)}$ and $L_{(1,1),(1,2)}$ described by
(a) $\sum_{i}\left(\right.$ No. of $i$-cells in $\left.M_{(1,1),(1,1,1)}\right) v^{i}=v^{r-1}\left(\sum_{i=0}^{r-1} v^{i}\right)$,
(b) $\sum_{i}\left(\right.$ No. of $i$-cells in $\left.M_{(1,1),(1,2)}\right) v^{i}=\sum_{0 \leq i<j \leq r-1}\left(v^{i+j-1}+v^{i+j}\right)$,
(c) $\sum_{i}\left(\right.$ No. of $i$-cells in $\left.L_{(1,1),(1,1,1)}\right) v^{i}=\sum_{i=0}^{r-1} v^{i} \quad$ and
(d) $\sum_{i}\left(\right.$ No. of $i$-cells in $\left.L_{(1,1),(1,2)}\right) v^{i}=\sum_{0 \leq i<j \leq r-1}\left(v^{i+j-1}+v^{i+j}\right)$.

\section{4.b. The Hilbert function strata of $\operatorname{Hilb}^{n}\left(\mathbb{A}^{2}, 0\right)$.}

In the case of $\operatorname{Hilb}^{n}\left(\mathbb{A}^{2}, 0\right)$, many results about the varieties $M_{T}$ and $L_{T}$ have been obtained in [Br], [Iar], [Gö1] and [Gö2]. Let us just list some of these results here.

Proposition 3.4.3 ([Iar, Prop. 1.6, Thm. 2.11, Thm. 2.12, Thm. 3.13]).

(1) $M_{T}$ and $L_{T}$ are non-empty if and only if $t_{0}=1$ and $t_{i} \leq t_{i-1}$ for all $i \geq d$, where $d$ is the initial degree of $T$. 
(2) $M_{T}$ and $L_{T}$ are smooth and irreducible. $L_{T}$ is projective of dimension

$$
\operatorname{dim}\left(L_{T}\right)=\sum_{j \geq d}\left(t_{j-1}-t_{j}+1\right)\left(t_{j}-t_{j+1}\right) .
$$

(3) $\rho_{T}: M_{T} \rightarrow L_{T}$ is a locally trivial fibre bundle in the Zariski topology, whose fibre is an affine space $\mathbb{A}^{n(T)}$ of dimension

$$
n(T)=n-\sum_{j \geq d}\left(t_{j-1}-t_{j}+1\right)\left(\frac{1}{2} t_{j-1}+\frac{1}{2} t_{j}-t_{j+1}\right) .
$$

Under a $\mathbb{C}^{*}$-action on $\operatorname{Hilb}^{n}\left(\mathbb{A}^{2}, 0\right)$ induced by one of the one-parameter subgroups described in Lemma 3.4.1, $L_{T}$ is $\mathbb{C}^{*}$-invariant. Since $L_{T}$ is smooth and projective, one obtains by Theorem 3.0.2 a cellular decomposition for $L_{T}$ and since the $\mathbb{C}^{*}$-action on $L_{T}$ is a restriction to $L_{T}$ of the $\mathbb{C}^{*}$-action on $M_{T}$ [Lemma 3.4.1.2], the cells of $L_{T}$ are the intersections of $L_{T}$ with the cells of $M_{T}$ in the cellular decomposition of $M_{T}$ described in Lemma 3.4.1.1. The number of $i$-cells in both $L_{T}$ and $M_{T}$ can be expressed in terms of Young diagrams.

Recall that to each partition of $n$, we may associate a Young diagram with $n$ boxes. Each box of the Young diagram can be represented by a pair of non-negative integers as illustrated in the following picture:

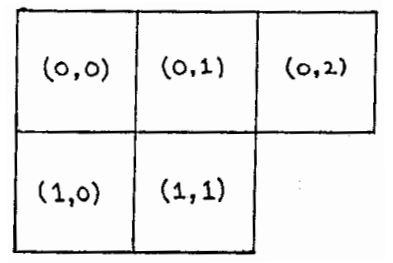

\section{Figure 8.}

The diagonal sequence associated to the Young diagram $\Gamma$ is $T(\Gamma)=$ $\left(t_{0}(\Gamma), t_{1}(\Gamma), \ldots\right)$, where $t_{i}(\Gamma):=|\{(l, k) \in \Gamma: l+k=i\}|$. So, it is the sequence of numbers of points on the diagonals of $\Gamma$.

Example. For the Young diagram $\Gamma$ in Figure 8, we have $t_{0}(\Gamma)=1, t_{1}(\Gamma)=$ $2, t_{2}(\Gamma)=2$ and $t_{i}(\Gamma)=0$ for $i \geq 3$.

Let $(u, v) \in \Gamma$. Then, the hook difference $h_{u, v}(\Gamma)$ is

$$
h_{u, v}(\Gamma)=|\{(u, l) \in \Gamma: l>v\}|-|\{(k, v) \in \Gamma: k>u\}| .
$$

In other words, $h_{u, v}(\Gamma)$ is the difference between the number of points in $\Gamma$ in the same row to the right of $(u, v)$ and the number of points in the same column below $(u, v)$. 
Example. For the Young diagram $\Gamma$ below, the hook differences $h_{u, v}(\Gamma)$ are given by

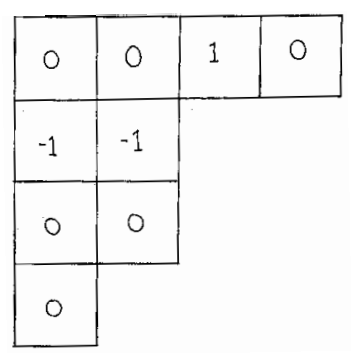

Figure 9.

Theorem 3.4.4 ([Gö2, Thm.2.2.7]). Let $T=\left(t_{i}\right)_{i \geq 0}$ be a sequence of nonnegative integers with $|T|=n$. Then, we have for $X=L_{T}$ and $X=M_{T}$ :-

(1) $X$ has a cellular decomposition; the map $A_{*}(X) \rightarrow H_{*}(X)$ is an isomorphism and $H_{*}(X)$ is free.

$$
b_{2 i}\left(M_{T}\right)=\left|\left\{\Gamma: \begin{array}{c}
T(\Gamma)=T \\
\left|\left\{(u, v) \in \Gamma: h_{u, v}(\Gamma) \in\{0,1\}\right\}\right|=n-i
\end{array}\right\}\right| .
$$

$$
b_{2 i}\left(L_{T}\right)=\left|\left\{\Gamma: \begin{array}{c}
T(\Gamma)=T \\
\left|\left\{(u, v) \in \Gamma: h_{u, v}(\Gamma)=-1\right\}\right|=i
\end{array}\right\}\right| .
$$

In particular, the Euler numbers are

$$
\chi\left(M_{T}\right)=\chi\left(L_{T}\right)=|\{\Gamma: T(\Gamma)=T\}| .
$$

In fact, Göttsche shows that if $\Gamma$ is the Young diagram associated (see Section 2.5) to the invariant ideal $I$ of $\mathbb{C}[x, y]=\left[Y_{1} / Y_{0}, Y_{2} / Y_{0}\right]$ corresponding to a $\mathbb{C}^{*}$-fixed point of $\operatorname{Hilb}^{n}\left(\mathbb{A}^{2}, 0\right) \subset \operatorname{Hilb}^{n}\left(\mathbb{A}^{2}\right)=\operatorname{Hilb}^{n}(\operatorname{Spec} \mathbb{C}[x, y])$, then $I$ lies in $L_{T(\Gamma)} \subset M_{T(\Gamma)}$ and the dimension of the positive part of the tangent space to $\operatorname{Hilb}^{n}\left(\mathbb{A}^{2}\right)$ at $I$ is equal to

$$
n-\left|\left\{(u, v) \in \Gamma: h_{u, v}(\Gamma) \in\{0,1\}\right\}\right|,
$$

while the dimension of the positive part of the tangent space to $L_{T(\Gamma)}$ at $I$ is equal to

$$
\left|\left\{(u, v) \in \Gamma: h_{u, v}(\Gamma)=-1\right\}\right| .
$$

Göttsche points out that under the canonical isomorphism identifying the tangent space to $\operatorname{Hilb}^{n}\left(\mathbb{A}^{2}\right)$ at $I$ with $V=\operatorname{Hom}_{R}(I, R / I)$, where $R=\mathbb{C}[x, y]$, 
the tangent space $V_{1}$ to $M_{T}$ at $I$ is identified with the homomorphisms which preserve or increase degree and the tangent space $V_{0}$ to $L_{T}$ at $I$ is identified with the homomorphisms which preserve degree. We have

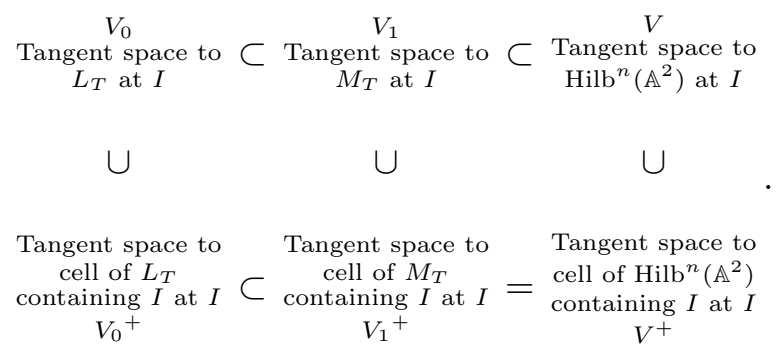

Let us rephrase the situation in the language of Section 2.5. Recall that $\operatorname{Hom}_{R}(I, R / I)$ is generated by the subset $\mathcal{S}$ consisting of elements of pure weight which take canonical generators of $I$ either to zero or to monomials modulo $I$. A homomorphism in $\mathcal{S}$ moves any two canonical generators not taken to zero by the same horizontal and vertical translation.

\section{Note 3.4.5.}

(i) $\quad V_{1}$ is the subset of $V$ generated by the homomorphisms $f$ in $\mathcal{S}$ which take any canonical generator $\alpha$ for which $f(\alpha) \neq 0$ to a box in the diagonal containing $\alpha$ or to a box in a larger diagonal.

(ii) $V_{0}$ is the subset of $V$ generated by the homomorphisms $f$ in $\mathcal{S}$ which take any canonical generator $\alpha$ for which $f(\alpha) \neq 0$ to a box in the diagonal containing $\alpha$.

(iii) $V_{1}^{+}$is the subset of $V$ generated by the homomorphisms $f$ in $\mathcal{S}$ which take any canonical generator $\alpha$ for which $f(\alpha) \neq 0$ to a box in the diagonal containing $\alpha$ lying in a lower row than $\alpha$ or to a box in a larger diagonal.

(iv) $V_{0}^{+}$is the subset of $V$ generated by the homomorphisms $f$ in $\mathcal{S}$ which take any canonical generator $\alpha$ for which $f(\alpha) \neq 0$ to a box in the diagonal containing $\alpha$ lying in a lower row than $\alpha$.

Before turning to the case $\mathcal{Z}_{n-1, n}\left(\mathbb{A}^{2}, 0\right)$, we also need to look more closely at the work [Iar] of Iarrobino; we present here some of his methods and results in the language of this paper. The only point that might lead to confusion is the fact that Iarrobino uses the ordering of monomials $0<1<$ $y<x<y^{2}<y x<x^{2}<\ldots$ whereas we use the ordering $0<1<x<y<$ $x^{2}<x y<y^{2}<\ldots$ in keeping with the rest of our work. The reader will have to swap the symbols $x$ and $y$ around when referring to [Iar].

Let $\Gamma_{T}$ be the unique Young diagram which has diagonal sequence $T$ and satisfies

$$
j \geq 1 \text { and }(i, j) \in \Gamma_{T} \Rightarrow(i+1, j-1) \in \Gamma_{T} ;
$$


let $I_{T}$ be the $\mathbb{C}^{*}$-fixed point in $L_{T} \subset M_{T}$ corresponding to this Young diagram. If $d$ is the initial degree of $T$, the ideal $I_{T}$ has $d+1$ canonical generators. For the rest of this chapter (unlike in Chapter II), we will label the canonical generators $\alpha_{0}, \alpha_{1}, \ldots, \alpha_{d}$ from left to right, as in the picture below.

Example. If $T=(1,2,3,2), I_{T}$ is the ideal generated by

$$
\alpha_{0}=y^{4}, \alpha_{1}=x y^{3}, \alpha_{2}=x^{2} y \text { and } \alpha_{3}=x^{3} .
$$

$\Gamma_{T}$ is the diagram

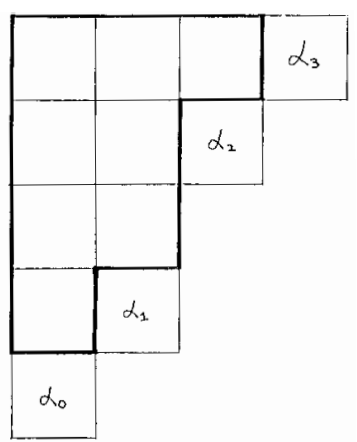

Figure 10.

With respect to a fixed system $(u=a x+b y, v=c x+d y)$ of parameters, let $\mathcal{P}((u, v), T)$ be the set of monomials $u^{j} v^{i}$ for which $(i, j) \in \Gamma_{T}$. Let

$$
M_{\mathcal{P}((u, v), T)}:=\left\{I \in M_{T}: I \cap\left(\begin{array}{c}
\text { linear span } \\
\text { of } \mathcal{P}((u, v), T)
\end{array}\right)=\{0\}\right\}
$$

and let

$$
L_{\mathcal{P}((u, v), T)}:=\left\{I \in L_{T}: I \cap\left(\begin{array}{c}
\text { linear span } \\
\text { of } \mathcal{P}((u, v), T)
\end{array}\right)=\{0\}\right\} .
$$

With respect to our chosen system $(x, y)=\left(Y_{1} / Y_{0}, Y_{2} / Y_{0}\right)$ of parameters, the set $M_{\mathcal{P}((x, y), T)}$ (resp. $\left.L_{\mathcal{P}((x, y), T)}\right)$ is exactly the cell of $M_{T}$ (resp. $L_{T}$ ) associated to the $\mathbb{C}^{*}$-fixed point $I_{T}$.

Proposition 3.4.6 ([Iar, Prop.3.2]). Let $N=\sum t_{j}\left(j+1-t_{j}\right)$. Then, any ideal $I \in M_{T}$ fails to be in $M_{\mathcal{P}((x, y-a x), T)}$ for at most $N$ values of a.

Remark 3.4.7. ([Iar, 3.A $]$ ). It follows that given any $N+1$ distinct points $a_{0}, a_{1}, \ldots, a_{N}$ in $\mathbb{C}$, the set $\left\{M_{\mathcal{P}\left(\left(x, y-a_{i} x\right), T\right)}: 0 \leq i \leq N\right\}$ is a finite open cover of $M_{T}$. This cover is connected because the $\mathbb{C}^{*}$-fixed point $I_{T}$ lies in 
each of the open sets $M_{\mathcal{P}\left(\left(x, y-a_{i} x\right), T\right)}$. Likewise, $L_{T}$ has a connected cover consisting of finitely many open sets which are isomorphic to $L_{\mathcal{P}((x, y), T)}$.

From now on, fix our system of parameters to be $(x, y)=\left(Y_{1} / Y_{0}, Y_{2} / Y_{0}\right)$ and write $\mathcal{P}(T)$ for $\mathcal{P}((x, y), T)$.

The Young diagram $\Gamma_{T}$ is identified with the set of monomials not in $I_{T}$ by letting the $(i, j)^{t h}$ box correspond to the monomial $x^{j} y^{i}$. Recall (see the definition preceeding Figure 4 in Chapter II) that there is a set of monomials $P_{\alpha}$ in $\Gamma_{T}$ associated to each canonical generator $\alpha$ of $I_{T}$. Let

$$
\begin{aligned}
& \Upsilon_{s}:=\left\{\beta \in P_{\alpha_{s}}: f_{\alpha_{s}, \beta} \text { preserves or increases degree }\right\} \\
& \Xi_{s}:=\left\{\beta \in P_{\alpha_{s}}: f_{\alpha_{s}, \beta} \text { preserves degree }\right\} \\
& \Delta_{s}:=\left\{\gamma \in \Gamma_{T} \backslash \Upsilon_{s}: \text { degree } \gamma \geq \text { degree } \alpha_{s}\right\} \text { and } \\
& \Lambda_{s}:=\left\{\gamma \in \Gamma_{T} \backslash \Xi_{s}: \text { degree } \gamma=\text { degree } \alpha_{s}\right\}
\end{aligned}
$$

let

$$
\begin{aligned}
& \Upsilon=\left\{(s, \beta): \beta \in \Upsilon_{s}\right\}, \quad \Xi=\left\{(s, \beta): \beta \in \Xi_{s}\right\} \\
& \Delta=\left\{(s, \beta): \beta \in \Delta_{s}\right\}
\end{aligned} \text { and } \Lambda=\left\{(s, \beta): \beta \in \Lambda_{s}\right\} .
$$

Since $\left\{f_{\alpha_{s}, \beta}:(s, \beta) \in \Upsilon\right\}$ is a basis for the tangent space to $M_{T}$ at $I_{T}$ and $\left\{f_{\alpha_{s}, \beta}:(s, \beta) \in \Xi\right\}$ is a basis for the tangent space to $L_{T}$ at $I_{T}$, we know that

$$
\operatorname{dim} M_{T}=|\Upsilon| \text { and } \operatorname{dim} L_{T}=|\Xi| .
$$

Proposition 3.4.8 ([Iar, Lemma 2.4, Prop. 2.5, Lemma 2.7, Prop. 2.8]).

(1) For each point $\left(a_{s, \beta}\right)_{(s, \beta) \in \Upsilon}$ in $\mathbb{A}^{\operatorname{dim} M_{T}}$, there is a unique sequence $\left(d_{s, \beta}\right)_{(s, \beta) \in \Delta}$ so that the ideal generated by the polynomials

$$
f_{s}=\alpha_{s}+\sum_{\beta \in \Upsilon_{s}} a_{s, \beta} \beta+\sum_{\beta \in \Delta_{s}} d_{s, \beta} \beta
$$

is in $M_{\mathcal{P}(T)}$. We can thus associate an ideal $I\left(a_{s, \beta}\right)$ to any point $\left(a_{s, \beta}\right)$ in $\mathbb{A}^{\operatorname{dim} M_{T}}$; the polynomials $f_{s}$ are known as the standard generators of this ideal. For each $\beta \in \Delta_{s}, d_{s, \beta}$ is a polynomial function of the $a_{t, \gamma}$ for which $t \leq s$ and $\beta \in \Upsilon_{t}$.

(2) The map from $\mathbb{A}^{\operatorname{dim} M_{T}}$ to $M_{\mathcal{P}(T)}$ which takes $\left(a_{s, \beta}\right)_{(s, \beta) \in \Upsilon}$ to $I\left(a_{s, \beta}\right)$ is an isomorphism of varieties.

(3) For each point $\left(a_{s, \beta}\right)_{(s, \beta) \in \Xi}$ in $\mathbb{A}^{\operatorname{dim} L_{T}}$, there is a unique sequence $\left(d_{s, \beta}\right)_{(s, \beta) \in \Lambda}$ so that the ideal generated by the polynomials

$$
f_{s}=\alpha_{s}+\sum_{\beta \in \Xi_{s}} a_{s, \beta} \beta+\sum_{\beta \in \Lambda_{s}} d_{s, \beta} \beta
$$


is in $L_{\mathcal{P}(T)}$. As before, this enables us to associate an ideal $I\left(a_{s, \beta}\right)$ to any point $\left(a_{s, \beta}\right)$ in $\mathbb{A}^{\operatorname{dim} L_{T}}$ and again, we call the polynomials $f_{s}$ the standard generators of this ideal. For each $\beta \in \Lambda_{s}, d_{s, \beta}$ is a polynomial function of the $a_{t, \gamma}$ for which $t \leq s$ and $\gamma \in \Xi_{t}$.

(4) The map from $\mathbb{A}^{\operatorname{dim} L_{T}}$ to $L_{\mathcal{P}(T)}$ which takes $\left(a_{s, \beta}\right)_{(s, \beta) \in \Xi}$ to $I\left(a_{s, \beta}\right)$ is an isomorphism of varieties.

(5) The inclusion map of $L_{\mathcal{P}(T)}$ in $M_{\mathcal{P}(T)}$ and the restriction of the projection map $\rho_{T}$ from $M_{\mathcal{P}(T)}$ to $L_{\mathcal{P}(T)}$ are given in coordinates in the obvious way. In particular, $M_{\mathcal{P}(T)}=L_{\mathcal{P}(T)} \times \mathbb{A}^{n(T)}$.

3.4.c. The Hilbert function strata of $\mathcal{Z}_{n-1, n}\left(\mathbb{A}^{2}, 0\right)$.

Finally, we turn to the case $\mathcal{Z}_{n-1, n}\left(\mathbb{A}^{2}, 0\right)$. We consider $\mathcal{Z}_{n-1, n}\left(\mathbb{A}^{2}, 0\right)$ under a $\mathbb{C}^{*}$-action induced by one of the one-parameter subgroups described in Lemma 3.4.1. Any fixed point of the action is a pair of invariant ideals $\left(I_{1}, I_{2}\right)$ of $\mathbb{C}[x, y]=\mathbb{C}\left[Y_{1} / Y_{0}, Y_{2} / Y_{0}\right]$ satisfying $I_{1} \supset I_{2}$; it corresponds to a pair of nested Young diagrams $\left(\Gamma_{1}, \Gamma_{2}\right)$ as shown in Figure 6.

Since any non-empty Hilbert function stratum $M_{T_{1}, T_{2}}$ must contain such a $\mathbb{C}^{*}$-fixed point $I \in L_{T_{1}, T_{2}}$, we have the analogue of the first part of Proposition 3.4.3: Given a pair of sequences $\left(T_{1}, T_{2}\right)=\left(\left(t_{0}^{1}, t_{1}^{1}, \ldots,\right),\left(t_{0}^{2}, t_{1}^{2}, \ldots,\right)\right)$ with $\left|T_{1}\right|=n-1$ and $\left|T_{2}\right|=n, M_{T_{1}, T_{2}}$ and $L_{T_{1}, T_{2}}$ are non-empty if and only if

(1) For $j \in\{1,2\}, t_{0}^{j}=1$ and $t_{i}^{j} \leq t_{i-1}^{j}$ for all $i \geq d_{0}^{j}$, where $d_{0}^{j}$ is the initial degree of $T_{j}$,

(2) There exists some $i$ for which $t_{i}^{1}+1=t_{i}^{2}$.

Consider the Young diagram $\Gamma_{2}$ and draw a diagonal line $\mathcal{L}$ through the box representing the monomial $\tilde{\alpha}$ which is in $I_{1}$ but not in $I_{2}$. Mark with the symbol $\diamond$ any box representing one of the canonical generators of $I_{2}$; mark with the symbol $\star$ any box which lies simultaneously in the same column below some $\diamond$ and in the same row to the right of some other $\diamond$, as illustrated in the following picture.

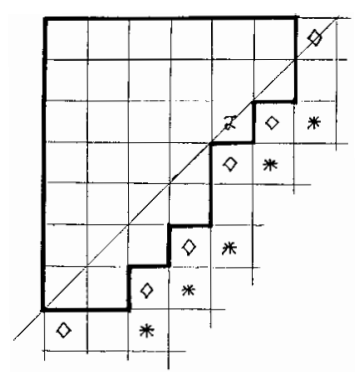

Figure 11. 
Let $\diamond \diamond\left(\Gamma_{1}, \Gamma_{2}\right)\left(\right.$ resp. $\left.\star \star\left(\Gamma_{1}, \Gamma_{2}\right)\right)$ be the number of symbols $\diamond$ (resp. $\left.\star\right)$ on the diagonal $\mathcal{L}$ or on a smaller diagonal than $\mathcal{L}$; let $\diamond\left(\Gamma_{1}, \Gamma_{2}\right)\left(\operatorname{resp} . \star\left(\Gamma_{1}, \Gamma_{2}\right)\right)$ be the number of symbols $\diamond$ (resp. $\star$ ) on the diagonal $\mathcal{L}$; let $\diamond \diamond^{+}\left(\Gamma_{1}, \Gamma_{2}\right)$ (resp. $\left.\star \star^{+}\left(\Gamma_{1}, \Gamma_{2}\right)\right)$ be the number of symbols $\diamond($ resp. $\star)$ on the diagonal $\mathcal{L}$ in a row higher than $\tilde{\alpha}$ or on a smaller diagonal than $\mathcal{L}$; let $\diamond^{+}\left(\Gamma_{1}, \Gamma_{2}\right)$ (resp. $\left.\star^{+}\left(\Gamma_{1}, \Gamma_{2}\right)\right)$ be the number of symbols $\diamond$ (resp. $\left.\star\right)$ on the diagonal $\mathcal{L}$ in a row higher than $\tilde{\alpha}$.

Lemma 3.4.9. Suppose that $\left(I_{1}, I_{2}\right) \in L_{T_{1}, T_{2}} \subset M_{T_{1}, T_{2}}$ is a $\mathbb{C}^{*}$-fixed point on $\mathcal{Z}_{n-1, n}\left(\mathbb{A}^{2}, 0\right)$ and $\left(\Gamma_{1}, \Gamma_{2}\right)$ is the associated pair of Young diagrams.

(1) The dimension of the tangent space to $M_{T_{1}, T_{2}}$ at $\left(I_{1}, I_{2}\right)$ is equal to

$$
\operatorname{dim} M_{T_{1}}+\diamond \diamond\left(\Gamma_{1}, \Gamma_{2}\right)-\star \star\left(\Gamma_{1}, \Gamma_{2}\right)
$$

(2) The dimension of the tangent space to $L_{T_{1}, T_{2}}$ at $\left(I_{1}, I_{2}\right)$ is equal to

$$
\operatorname{dim} L_{T_{1}}+\diamond\left(\Gamma_{1}, \Gamma_{2}\right)-\star\left(\Gamma_{1}, \Gamma_{2}\right) ;
$$

(3) The dimension of the positive part of the tangent space to $M_{T_{1}, T_{2}}$ at $\left(I_{1}, I_{2}\right)$ is equal to

$$
\operatorname{dim}\left(T_{I_{1}}\left(M_{T_{1}}\right)\right)^{+}+\diamond \diamond^{+}\left(\Gamma_{1}, \Gamma_{2}\right)-\star \star^{+}\left(\Gamma_{1}, \Gamma_{2}\right)
$$

(4) The dimension of the positive part of the tangent space to $L_{T_{1}, T_{2}}$ at $\left(I_{1}, I_{2}\right)$ is equal to

$$
\operatorname{dim}\left(T_{I_{1}}\left(L_{T_{1}}\right)\right)^{+}+\diamond^{+}\left(\Gamma_{1}, \Gamma_{2}\right)-\star^{+}\left(\Gamma_{1}, \Gamma_{2}\right) .
$$

Proof. We will just prove the first part of the lemma here as the proofs of the other parts are completely analogous.

Recall from Lemma 2.6.2 that we have an exact sequence

$$
\begin{aligned}
0 \rightarrow \operatorname{Ker}(\phi-\psi) \rightarrow \operatorname{Hom}_{R}\left(I_{1}, R / I_{1}\right) \oplus \operatorname{Hom}_{R}\left(I_{2}, R / I_{2}\right) \\
\qquad \stackrel{\phi-\psi}{\longrightarrow} \operatorname{Hom}_{R}\left(I_{2}, R / I_{1}\right) \rightarrow 0
\end{aligned}
$$

Let $\operatorname{Hom}_{R}\left(I_{2}, R / I_{1}\right)$ (resp. Ker $\psi$ ) be the subspace of $\operatorname{Hom}_{R}\left(I_{2}, R / I_{1}\right.$ ) (resp. $\operatorname{Ker} \bar{\psi}$ ) consisting of homomorphisms which preserve or increase degree. Restricting the exact sequence above to the homomorphisms which preserve or increase degree and applying the first part of Note 3.4.5, we have

$$
0 \rightarrow T_{\left(I_{1}, I_{2}\right)}\left(M_{T_{1}, T_{2}}\right) \rightarrow T_{I_{1}}\left(M_{T_{1}}\right) \oplus T_{I_{2}}\left(M_{T_{2}}\right) \rightarrow \underline{\operatorname{Hom}_{R}\left(I_{2}, R / I_{1}\right)} \rightarrow 0 .
$$


From Equations (2.6.1), (2.6.2), (2.6.3) and (2.6.4), we can express $\operatorname{Hom}_{R}\left(I_{2}, R / I_{1}\right)$ as a direct sum decomposition

$$
\left.\operatorname{Hom}_{R}\left(I_{2}, R / I_{1}\right)=\operatorname{Im} \psi \oplus \underset{i}{\oplus}\left\langle F_{i}\right\rangle\right) .
$$

We check that the number of homomorphisms $F_{i}$ which preserve or increase degree is equal to $\star \star\left(\Gamma_{1}, \Gamma_{2}\right)$. Hence,

(3.4.4) $\operatorname{dim} \underline{\operatorname{Hom}_{R}\left(I_{2}, R / I_{1}\right)}=\operatorname{dim} T_{I_{2}}\left(M_{T_{2}}\right)-\operatorname{dim} \underline{\operatorname{Ker} \psi}+\star \star\left(\Gamma_{1}, \Gamma_{2}\right)$.

Recall also that we constructed a basis $\left\{G_{i}\right\}$ for $\operatorname{Ker} \psi$ in the proof of Proposition 2.6.3, It is easy to see that the number of homomorphisms $G_{i}$ which preserve or increase degree is equal to $\diamond \diamond\left(\Gamma_{1}, \Gamma_{2}\right)$, and so

$$
\operatorname{dim} \underline{\operatorname{Ker} \psi}=\diamond \diamond\left(\Gamma_{1}, \Gamma_{2}\right) \text {. }
$$

The dimension of the tangent space to $M_{T_{1}, T_{2}}$ at $\left(I_{1}, I_{2}\right)$ can now be computed from the exact sequence (3.4.3) together with the Equations (3.4.4) and (3.4.5).

Let $\mathcal{L}_{i}$ be the $i^{\text {th }}$ diagonal and let $\Gamma$ be a Young diagram. We define $\diamond_{i}(\Gamma)$ (resp. $\left.\star_{i}(\Gamma)\right)$ to be the number of symbols $\diamond$ (resp. $\star$ ) lying on $\mathcal{L}_{i}$.

Lemma 3.4.10. Let $\Gamma$ be a Young diagram with the diagonal sequence $T(\Gamma)=\left(t_{0}, t_{1}, t_{2}, ..\right)$. Then,

$$
\diamond_{i}(\Gamma)-\star_{i}(\Gamma)= \begin{cases}0 & \text { if } i=0, \\ 2-t_{1} & \text { if } i=1, \\ \left(t_{i-1}-t_{i}\right)-\left(t_{i-2}-t_{i-1}\right) & \text { if } i \geq 2 .\end{cases}
$$

Proof. We use induction on the number of boxes $n$ in the Young diagram $\Gamma$. When $n=1$, the lemma is clearly true. So, let us assume that $\Gamma$ has $n$ boxes, where $n \geq 2$, and that the lemma holds for any Young diagram with less than $n$ boxes.

If $T(\Gamma)=\left(t_{0}, t_{1}, t_{2}, \ldots\right)$, let $k=\max \left\{i: t_{i} \neq 0\right\}$ and let $\Gamma^{\prime}$ be any Young diagram sitting inside $\Gamma$ with the diagonal sequence

$$
T\left(\Gamma^{\prime}\right)=\left(t_{0}, t_{1}, t_{2}, \ldots, t_{k-1}, t_{k}-1,0,0, \ldots\right) .
$$

One can verify, say by considering the cases listed in the beginning of Section 2.6 , that

$$
\begin{aligned}
\diamond_{i}(\Gamma)-\star_{i}(\Gamma) & =\diamond_{i}\left(\Gamma^{\prime}\right)-\star_{i}\left(\Gamma^{\prime}\right) \quad \text { if } 0 \leq i<k \text { or } k+2<i \\
\diamond_{k}(\Gamma)-\star_{k}(\Gamma) & =\diamond_{k}\left(\Gamma^{\prime}\right)-\star_{k}\left(\Gamma^{\prime}\right)-1 \\
\diamond_{k+1}(\Gamma)-\star_{k+1}(\Gamma) & =\diamond_{k+1}\left(\Gamma^{\prime}\right)-\star_{k+1}\left(\Gamma^{\prime}\right)+2 \\
\diamond_{k+2}(\Gamma)-\star_{k+2}(\Gamma) & =\diamond_{k+2}\left(\Gamma^{\prime}\right)-\star_{k+2}\left(\Gamma^{\prime}\right)-1 .
\end{aligned}
$$


For each $j$, we obtain by induction an expression for $\diamond_{j}\left(\Gamma^{\prime}\right)-\star_{j}\left(\Gamma^{\prime}\right)$ in terms of the diagonal sequence $\left\{t_{i}\right\}$. Plugging this expression for $\diamond_{j}\left(\Gamma^{\prime}\right)-\star_{j}\left(\Gamma^{\prime}\right)$ into the relevant equation in (3.4.6) then yields the required expression for $\diamond_{j}(\Gamma)-\star_{j}(\Gamma)$ in terms of the diagonal sequence $\left\{t_{i}\right\}$.

Following [Iar], we define $M_{\mathcal{P}\left(T_{1}\right), \mathcal{P}\left(T_{2}\right)}$ and $L_{\mathcal{P}\left(T_{1}\right), \mathcal{P}\left(T_{2}\right)}$ to be the sets

$$
M_{\mathcal{P}\left(T_{1}\right), \mathcal{P}\left(T_{2}\right)}:=\left\{\left(I_{1}, I_{2}\right) \in M_{T_{1}, T_{2}}: I_{1} \in M_{\mathcal{P}\left(T_{1}\right)} \text { and } I_{2} \in M_{\mathcal{P}\left(T_{2}\right)}\right\}
$$

and

$$
L_{\mathcal{P}\left(T_{1}\right), \mathcal{P}\left(T_{2}\right)}:=\left\{\left(I_{1}, I_{2}\right) \in L_{T_{1}, T_{2}}: I_{1} \in L_{\mathcal{P}\left(T_{1}\right)} \text { and } I_{2} \in L_{\mathcal{P}\left(T_{2}\right)}\right\}
$$

respectively.

We observe as before that $M_{\mathcal{P}\left(T_{1}\right), \mathcal{P}\left(T_{2}\right)}$ is exactly the cell of $M_{T_{1}, T_{2}}$ associated to the $\mathbb{C}^{*}$-fixed point $\left(I_{T_{1}}, I_{T_{2}}\right)$, while $L_{\mathcal{P}\left(T_{1}\right), \mathcal{P}\left(T_{2}\right)}$ is the set

$$
\left\{\left(I_{1}, I_{2}\right) \in L_{T_{1}, T_{2}}: \lim _{t \rightarrow 0}\left(t . I_{1}, t . I_{2}\right)=\left(I_{T_{1}}, I_{T_{2}}\right)\right\}
$$

The argument of Remark 3.4.7 shows that $M_{T_{1}, T_{2}}$ (resp. $L_{T_{1}, T_{2}}$ ) has a connected cover consisting of finitely many open sets which are isomorphic to $M_{\mathcal{P}\left(T_{1}\right), \mathcal{P}\left(T_{2}\right)}\left(\right.$ resp. $\left.L_{\mathcal{P}\left(T_{1}\right), \mathcal{P}\left(T_{2}\right)}\right)$.

Proposition 3.4.11. Every Hilbert function stratum $M_{T_{1}, T_{2}}$ of $\mathcal{Z}_{n-1, n}\left(\mathbb{A}^{2}, 0\right)$ is smooth and irreducible. If we write $T_{1}=\left(t_{0}, t_{1}, \ldots, t_{k-1}, t_{k}\right.$, $\left.t_{k+1}, \ldots\right)$ and $T_{2}=\left(t_{0}, t_{1}, \ldots, t_{k-1}, t_{k}+1, t_{k+1}, \ldots\right)$ and let $d$ be the initial degree of $T_{1}$, then we have

$$
\operatorname{dim} M_{T_{1}, T_{2}}=(n-1)-\sum_{j \geq d}\left(t_{j-1}-t_{j}\right)\left(t_{j-1}-t_{j}+1\right) / 2+\left(t_{k-1}-t_{k}\right) .
$$

Proof. It is clear that $M_{T_{1}, T_{2}}$ is smooth and irreducible as it has a connected cover of finitely many smooth open subsets. To obtain the formula for its dimension, observe that

$$
\operatorname{dim} M_{T_{1}, T_{2}}=\operatorname{dim} M_{T_{1}}+\left(t_{k-1}-t_{k}\right)
$$

by Lemma 3.4.9.1 and Lemma 3.4.10, while

$$
\operatorname{dim} M_{T_{1}}=(n-1)-\sum_{j \geq d}\left(t_{j-1}-t_{j}\right)\left(t_{j-1}-t_{j}+1\right) / 2
$$

by Proposition 3.4.3. 
Proposition 3.4.12. The space $L_{T_{1}, T_{2}}$ is an irreducible smooth projective variety. With the notation of Proposition 3.4.11, the dimension of $L_{T_{1}, T_{2}}$ is equal to

$$
\sum_{j \geq d}\left(t_{j-1}-t_{j}+1\right)\left(t_{j}-t_{j+1}\right)+\left(t_{k-1}-t_{k}-1\right)-\left(t_{k-2}-t_{k-1}\right) .
$$

Proof. It is clear that $L_{T_{1}, T_{2}}$ is a closed subset of the projective variety $L_{T_{1}} \times$ $L_{T_{2}}$. Since $L_{T_{1}, T_{2}}$ has a connected cover consisting of finitely many open subsets isomorphic to $L_{\mathcal{P}\left(T_{1}\right), \mathcal{P}\left(T_{2}\right)}$ and since the set

$$
L_{\mathcal{P}\left(T_{1}\right), \mathcal{P}\left(T_{2}\right)}:=\left\{\left(I_{1}, I_{2}\right) \in L_{T_{1}, T_{2}}: \lim _{t \rightarrow 0}\left(t . I_{1}, t . I_{2}\right)=\left(I_{T_{1}}, I_{T_{2}}\right)\right\}
$$

is obviously connected, we need only show that $L_{\mathcal{P}\left(T_{1}\right), \mathcal{P}\left(T_{2}\right)}$ is a smooth variety whose dimension is given by expression (3.4.7).

Let $\tilde{d}$ be the initial degree of $T_{2}$; let $\left\{\alpha_{0}, \alpha_{1}, \ldots, \alpha_{d}\right\}$ (resp. $\left\{\tilde{\alpha}_{0}, \tilde{\alpha}_{1}, \ldots\right.$, $\left.\left.\tilde{\alpha}_{\tilde{d}}\right\}\right)$ be the set of canonical generators of $I_{T_{1}}$ (resp. $I_{T_{2}}$ ) labelled as in Figure 10. Let $\Xi_{s}$ (resp. $\tilde{\Xi}_{s}$ ) be the set of elements $\beta$ in $P_{\alpha_{s}} \subset \Gamma_{T_{1}}$ (resp. $P_{\tilde{\alpha}_{s}} \subset \Gamma_{T_{2}}$ ) for which the homomorphism $f_{\alpha_{s}, \beta}$ (resp. $f_{\tilde{\alpha}_{s}, \beta}$ ) preserves degree and let $\Xi=\left\{(s, \beta): \beta \in \Xi_{s}\right\}$ (resp. $\tilde{\Xi}=\left\{(s, \beta): \beta \in \tilde{\Xi}_{s}\right\}$ ). By Proposition 3.4.8.4, $L_{\mathcal{P}\left(T_{1}\right)}$ (resp. $\left.L_{\mathcal{P}\left(T_{2}\right)}\right)$ is an affine space whose point with coordinates $\left(a_{s, \beta}\right)_{(s, \beta) \in \Xi}\left(\operatorname{resp} . \quad\left(\tilde{a}_{s, \beta}\right)_{(s, \beta) \in \tilde{\Xi}}\right)$ represents the ideal $I\left(a_{s, \beta}\right)$ (resp. $\left.I\left(\tilde{a}_{s, \beta}\right)\right)$.

There are three cases to consider: the case where $\tilde{d}=d<k$, the case where $\tilde{d}=d+1$ and the case where $\tilde{d}=d=k$.

Case 1. $\tilde{d}=d<k$. Recall that if $f_{0}, f_{1}, \ldots, f_{d}$ are the standard generators of $I\left(a_{s, \beta}\right)$, then

$$
f_{s}=\alpha_{s}+\sum_{\beta \in \mathcal{P}\left(T_{1}\right)} A_{s, \beta} \beta,
$$

where $A_{s, \beta}$ is a polynomial in the $a_{t, \gamma}$ for which $t \leq s$ and $\gamma \in \Xi_{t}$; in the case where $\beta \in \Xi_{s}, A_{s, \beta}=a_{s, \beta}$, and in the case where $\operatorname{deg} \beta \neq \operatorname{deg} \alpha_{s}, A_{s, \beta}=0$.

To each pair consisting of a point $\left(a_{s, \beta}\right)$ in $L_{\mathcal{P}\left(T_{1}\right)}$ and a $\left(t_{k-1}-t_{k}-1\right)$-tuple $\left(k_{p}\right)_{t_{k}<p<t_{k-1}}$, we associate an ideal $J\left(\left(a_{s, \beta}\right),\left(k_{p}\right)\right)$ of $\mathbb{C}[x, y]$ as follows. If $f_{0}$, $f_{1}, \ldots, f_{d}$ are the standard generators of $I\left(a_{s, \beta}\right)$, let $J\left(\left(a_{s, \beta}\right),\left(k_{p}\right)\right)$ be the ideal generated by the polynomials $f_{0}^{\prime}, f_{1}^{\prime}, \ldots, f_{d}^{\prime}$, where

$$
f_{i}^{\prime}= \begin{cases}f_{i} & \text { if } i<t_{k}, \\ y f_{t_{k}}-\sum_{j=t_{k+1}}^{t_{k}-1} a_{t_{k}, \alpha_{j} / y} f_{j} & \text { if } i=t_{k}, \\ f_{i}+k_{i} f_{t_{k}} & \text { if } t_{k}<i<t_{k-1}, \\ f_{i} & \text { if } i \geq t_{k-1} .\end{cases}
$$


Writing $\alpha_{s}$ as $x^{s} y^{l_{s}}$ and letting $m_{s}=l_{s-1}-l_{s}$, we have:

Lemma 3.4.13. For $t_{k-1}+1 \leq r \leq t_{k-2}$, there is a polynomial $g_{r}$ in the coordinates $a_{s, \beta}$ and $k_{p}$ such that

$$
y^{m_{r}} f_{r}-x f_{r-1}=g_{r}\left(\left(a_{s, \beta}\right),\left(k_{p}\right)\right) f_{t_{k}} \bmod \left\langle f_{t_{k}+1}^{\prime}, \ldots, f_{r-1}^{\prime}\right\rangle .
$$

We will return to the proof of this lemma when we are done with proving Proposition 3.4.12.

Define $H$ to be the closed subvariety of $L_{\mathcal{P}\left(T_{1}\right)} \times \mathbb{A}^{t_{k-1}-t_{k}-1}$ satisfying the equations

$$
g_{r}\left(\left(a_{s, \beta}\right),\left(k_{p}\right)\right)=0 \text { for } t_{k-1}+1 \leq r \leq t_{k-2} .
$$

We claim that if $\left(\left(a_{s, \beta}\right),\left(k_{p}\right)\right)$ lies in $H$, the pair of ideals $\left(I\left(a_{s, \beta}\right), J\left(\left(a_{s, \beta}\right)\right.\right.$, $\left.\left.\left(k_{p}\right)\right)\right)$ is in $L_{\mathcal{P}\left(T_{1}\right), \mathcal{P}\left(T_{2}\right)}$ :

(1) It is clear that $J\left(\left(a_{s, \beta}\right),\left(k_{p}\right)\right)$ is contained in $I\left(a_{s, \beta}\right)$; in fact, we have

$$
\left\langle J\left(\left(a_{s, \beta}\right),\left(k_{p}\right)\right), f_{t_{k}}\right\rangle=I\left(a_{s, \beta}\right) .
$$

Since $x f_{t_{k}}$ and $y f_{t_{k}}$ are both in $J\left(\left(a_{s, \beta}\right),\left(k_{p}\right)\right)$, either $J\left(\left(a_{s, \beta}\right),\left(k_{p}\right)\right)=$ $I\left(a_{s, \beta}\right)$ or $J\left(\left(a_{s, \beta}\right),\left(k_{p}\right)\right) \in L_{T_{2}}$. (To see that $x f_{t_{k}}$ is in $J\left(\left(a_{s, \beta}\right),\left(k_{p}\right)\right.$ ) requires a short argument analogous to the proof of Lemma 3.4.13.)

(2) That $J\left(\left(a_{s, \beta}\right),\left(k_{p}\right)\right)$ is in $L_{\mathcal{P}\left(T_{2}\right)}$ is then a consequence of the following lemma, which we will prove later.

Lemma 3.4.14. If $\left(\left(a_{s, \beta}\right),\left(k_{p}\right)\right) \in H$,

$$
\left\langle f_{0}^{\prime}, f_{1}^{\prime}, \ldots, f_{r}^{\prime}\right\rangle \cap\left(\begin{array}{c}
\text { linear span } \\
\text { of } \mathcal{P}\left(T_{2}\right)
\end{array}\right)=\{0\} \quad \text { for any } r \leq d .
$$

Regarded as a point in $L_{\mathcal{P}\left(T_{2}\right)}$, the ideal $J\left(\left(a_{s, \beta}\right),\left(k_{p}\right)\right)$ has coordinates $\left(\tilde{a}_{s, \beta}\right)$ specified by

$$
\tilde{a}_{s, \beta}= \begin{cases}A_{s, \beta} & \text { if } s<t_{k}, \\ A_{t_{k}, \beta / y}-\sum_{j=t_{k+1}}^{t_{k}-1} A_{t_{k}, \alpha_{j} / y} A_{j, \beta} & \text { if } s=t_{k}, \\ A_{s, \beta}+k_{s} A_{t_{k}, \beta} & \text { if } t_{k}<s<t_{k-1} \text { and } \beta \neq \alpha_{t_{k}}, \\ k_{s} & \text { if } t_{k}<s<t_{k-1} \text { and } \beta=\alpha_{t_{k}}, \\ A_{s, \beta} & \text { if } t_{k-1} \leq s .\end{cases}
$$


Therefore, the locus of such pairs of ideals $\left(I\left(a_{s, \beta}\right), J\left(\left(a_{s, \beta}\right),\left(k_{p}\right)\right)\right)$ is the closed subvariety $Y$ of $L_{\mathcal{P}\left(T_{1}\right), \mathcal{P}\left(T_{2}\right)} \subset L_{\mathcal{P}\left(T_{1}\right)} \times L_{\mathcal{P}\left(T_{2}\right)}$ consisting of points whose coordinates $\left(\left(a_{s, \beta}\right),\left(\tilde{a}_{s, \beta}\right)\right)$ satisfy the equations

$$
g_{r}\left(\left(a_{s, \beta}\right),\left(\tilde{a}_{p, \alpha_{t_{k}}}\right)\right)=0 \quad \text { for } t_{k-1}+1 \leq r \leq t_{k-2},
$$

together with the equations

$$
\begin{array}{ll}
\tilde{a}_{s, \beta}=A_{s, \beta} & \text { for } s<t_{k} \text { and } \beta \in \tilde{\Xi}_{s}, \\
\tilde{a}_{s, \beta}=A_{t_{k}, \beta / y}-\sum_{j=t_{k+1}}^{t_{k}-1} A_{t_{k}, \alpha_{j} / y} A_{j, \beta} & \text { for } s=t_{k} \text { and } \beta \in \tilde{\Xi}_{s}, \\
\tilde{a}_{s, \beta}=A_{s, \beta}+\tilde{a}_{s, \alpha_{t_{k}}} A_{t_{k}, \beta} & \text { for } t_{k}<s<t_{k-1} \text { and } \beta \in \tilde{\Xi}_{s} \backslash\left\{\alpha_{t_{k}}\right\}, \\
\tilde{a}_{s, \beta}=A_{s, \beta} & \text { for } t_{k-1} \leq s \text { and } \beta \in \tilde{\Xi}_{s} .
\end{array}
$$

Because the morphism from $H$ to $Y$ associating the pair of ideals $\left(I\left(a_{s, \beta}\right)\right.$, $\left.J\left(\left(a_{s, \beta}\right),\left(k_{p}\right)\right)\right)$ to the point $\left(\left(a_{s, \beta}\right),\left(k_{p}\right)\right)$ in $H$ is an isomorphism, we know that every component of $Y$ has dimension at least

$$
\operatorname{dim} L_{T_{1}}+\left(t_{k-1}-t_{k}-1\right)-\left(t_{k-2}-t_{k-1}\right) .
$$

On the other hand, by Lemma 3.4.9.2 and Lemma 3.4.10, the tangent space at any $\mathbb{C}^{*}$-fixed point of $L_{T_{1}, T_{2}}$ has dimension

$$
\operatorname{dim} L_{T_{1}}+\left(t_{k-1}-t_{k}-1\right)-\left(t_{k-2}-t_{k-1}\right) .
$$

Since the closure of the $\mathbb{C}^{*}$-orbit of any point of $L_{T_{1}, T_{2}}$ must contain one of these fixed points, no tangent space to $L_{T_{1}, T_{2}}$ can have a larger dimension. This, together with (3.4.10) implies that $Y=L_{\mathcal{P}\left(T_{1}\right), \mathcal{P}\left(T_{2}\right)}$ is a smooth variety whose dimension is given by expression (3.4.11). By Proposition 3.4.3.2,

$$
\operatorname{dim} L_{T_{1}}=\sum_{j \geq d}\left(t_{j-1}-t_{j}+1\right)\left(t_{j}-t_{j+1}\right),
$$

so (3.4.11) is equal to (3.4.7) as required.

Case 2. $\tilde{d}=d+1$. This case can only occur when $d=k=t_{k}$. Hence, $\left(t_{k-1}-t_{k}-1\right)-\left(t_{k-2}-t_{k-1}\right)=0$ and we are required to demonstrate that $L_{\mathcal{P}\left(T_{1}\right), \mathcal{P}\left(T_{2}\right)}$ is a smooth variety whose dimension is equal to that of $L_{T_{1}}$.

This follows because we can show as in the previous case that the projection map $\varpi: L_{\mathcal{P}\left(T_{1}\right), \mathcal{P}\left(T_{2}\right)} \rightarrow L_{\mathcal{P}\left(T_{1}\right)}$ is surjective and we know by Lemma 3.4.9.2 and Lemma 3.4.10 that the dimension of every tangent space to $L_{\mathcal{P}\left(T_{1}\right), \mathcal{P}\left(T_{2}\right)}$ is no more than the dimension of $L_{\mathcal{P}\left(T_{1}\right)}$. 
Case 3. $\tilde{d}=d=k$. Again, a similar but simpler argument to that in Case 1 shows that the projection map $\varpi: L_{\mathcal{P}\left(T_{1}\right), \mathcal{P}\left(T_{2}\right)} \rightarrow L_{\mathcal{P}\left(T_{1}\right)}$ is surjective with each fibre having dimension at least $t_{k-1}-t_{k}$. This, together with Lemma 3.4.9.2 and Lemma 3.4.10, yields

$$
\operatorname{dim} L_{\mathcal{P}\left(T_{1}\right), \mathcal{P}\left(T_{2}\right)}=\operatorname{dim} L_{\mathcal{P}\left(T_{1}\right)}+\left(t_{k-1}-t_{k}\right)
$$

as needed.

Proof of Lemma 3.4.13. The procedure for obtaining the polynomial $g_{r}\left(\left(a_{s, \beta}\right),\left(k_{p}\right)\right)$ is as follows:

(1) Write $E_{1}=y^{m_{r}} f_{r}-x f_{r-1}$ as $\sum_{\text {monomials } \gamma} h_{\gamma}\left(a_{s, \beta}\right) \gamma$; then, eliminate monomials divisible by $\alpha_{r-1}$ from $E_{1}$ by subtracting from $E_{1}$ a suitable multiple $h_{r-1} f_{r-1}^{\prime}$ of $f_{r-1}^{\prime}$ to obtain $E_{2}=E_{1}-h_{r-1} f_{r-1}^{\prime}$.

(2) Having obtained $E_{i}$, eliminate monomials divisible by $\alpha_{r-i}$ from $E_{i}$ by subtracting from $E_{i}$ a suitable multiple $h_{r-i} f_{r-i}^{\prime}$ of $f_{r-i}^{\prime}$ to obtain $E_{i+1}=E_{i}-h_{r-i} f_{r-i}^{\prime}$.

(3) After $r-t_{k}-1$ steps, we obtain $E_{r-t_{k}}$. $E_{r-t_{k}}$ lies in the linear span of $\mathcal{P}\left(T_{1}\right)$ and $\alpha_{t_{k}}$, and satisfies

$$
E_{r-t_{k}}=y^{m_{r}} f_{r}-x f_{r-1} \quad \bmod \left\langle f_{t_{k}+1}^{\prime}, \ldots, f_{r-1}^{\prime}\right\rangle .
$$

The process produces polynomials $G_{\beta}$ and $g$ in the coordinates $a_{s, \beta}$ and $k_{p}$ so that

$$
E_{r-t_{k}}=g f_{t_{k}}+\sum_{\beta \in \mathcal{P}\left(T_{1}\right)} G_{\beta} \beta
$$

Since $E_{r-t_{k}}$ and $f_{t_{k}}$ are both in $I\left(a_{s, \beta}\right)$ and $I\left(a_{s, \beta}\right) \cap\left(\begin{array}{c}\text { linear span } \\ \text { of } \mathcal{P}\left(T_{1}\right)\end{array}\right)=\{0\}$, the polynomials $G_{\beta}$ are identically zero and $E_{r-t_{k}}=g f_{t_{k}}$.

Proof of Lemma 3.4.14. If $\left\langle f_{0}^{\prime}, f_{1}^{\prime}, \ldots, f_{r}^{\prime}\right\rangle \cap\left(\begin{array}{c}\text { linear span } \\ \text { of } \mathcal{P}\left(T_{2}\right)\end{array}\right) \neq\{0\}$, there must exist some

$$
g=f_{t_{k}}+\sum_{\substack{\operatorname{deg} \beta=k \\ \beta \in \mathcal{P}\left(T_{1}\right)}} \lambda_{\beta} \beta
$$

in the ideal generated by $f_{0}^{\prime}, f_{1}^{\prime}, \ldots, f_{r}^{\prime}$. This is clearly not possible if $r \leq t_{k}$ since the polynomials $f_{0}^{\prime}, f_{1}^{\prime}, \ldots, f_{t_{k}}^{\prime}$ all have degrees larger than $k$.

We proceed to prove the lemma by induction on $r$. Suppose that

$$
\left\langle f_{0}^{\prime}, f_{1}^{\prime}, \ldots, f_{r-1}^{\prime}\right\rangle \cap\left(\begin{array}{c}
\text { linear span } \\
\text { of } \mathcal{P}\left(T_{2}\right)
\end{array}\right)=\{0\}
$$


and assume for contradiction that

$$
\left\langle f_{0}^{\prime}, f_{1}^{\prime}, \ldots, f_{r}^{\prime}\right\rangle \cap\left(\begin{array}{c}
\text { linear span } \\
\text { of } \mathcal{P}\left(T_{2}\right)
\end{array}\right) \neq\{0\} .
$$

Case 1. $t_{k}<r<t_{k-1}$. For (ii) to hold, there must exist complex numbers $\mu_{s}, \lambda_{\beta}$ and $\lambda$ such that

$$
\lambda f_{t_{k}}+\sum_{\substack{\operatorname{deg} \beta=k \\ \beta \in \mathcal{P}\left(T_{1}\right)}} \lambda_{\beta} \beta=f_{r}^{\prime}+\sum_{s=t_{k}+1}^{r-1} \mu_{s} f_{s}^{\prime} .
$$

As all terms in the above equation apart from $f_{r}^{\prime}$ are divisible by $y^{l_{r-1}}$, we have a contradiction.

Case 2. $r=t_{k-1}$. The same arguments lead to a contradiction.

Case 3. $t_{k-1}<r \leq t_{k-2}$. For (ii) to hold, there must exist complex numbers $\lambda_{\beta}$, polynomials $h_{s}$ and a polynomial $h$ of degree $m_{r}$ such that

$$
f_{t_{k}}+\sum_{\substack{\operatorname{deg} \beta=k \\ \beta \in \mathcal{P}\left(T_{1}\right)}} \lambda_{\beta} \beta=h f_{r}^{\prime}+\sum_{s=0}^{r-1} h_{s} f_{s}^{\prime} .
$$

Since $y^{l_{r-1}}$ divides the left hand side of the equation as well as the sum $\sum_{s=0}^{r-1} h_{s} f_{s}^{\prime}$, it must also divide $h f_{r}^{\prime}$. In particular, $h=\lambda y^{m_{r}}$ and

$$
f_{t_{k}}+\sum_{\substack{\operatorname{deg} \beta=k \\ \beta \in \mathcal{P}\left(T_{1}\right)}} \lambda_{\beta} \beta=\lambda\left(y^{m_{r}} f_{r}^{\prime}-x f_{r-1}^{\prime}\right)+\lambda x f_{r-1}^{\prime}+\sum_{s=0}^{r-1} h_{s} f_{s}^{\prime} .
$$

However, the fact that the point $\left(\left(a_{s, \beta}\right),\left(k_{p}\right)\right)$ lies in $H$ implies that

$$
y^{m_{r}} f_{r}-x f_{r-1} \in\left\langle f_{0}^{\prime}, f_{1}^{\prime}, \ldots, f_{r-1}^{\prime}\right\rangle,
$$

so we obtain

$$
f_{t_{k}}+\sum_{\substack{\operatorname{deg} \beta=k \\ \beta \in \mathcal{P}\left(T_{1}\right)}} \lambda_{\beta} \beta \in\left\langle f_{0}^{\prime}, f_{1}^{\prime}, \ldots, f_{r-1}^{\prime}\right\rangle,
$$

contradicting (i).

Case 4. $r>t_{k-2}$. Again, for (ii) to hold, there must exist polynomials $h$ and $h_{s}$, and complex numbers $\lambda_{\beta}$ such that

$$
f_{t_{k}}+\sum_{\substack{\operatorname{deg} \beta=k \\ \beta \in \mathcal{P}\left(T_{1}\right)}} \lambda_{\beta} \beta=h\left(y^{m_{r}} f_{r}-x f_{r-1}\right)+\sum_{s=0}^{r-1} h_{s} f_{s}^{\prime} .
$$


To obtain a contradiction as before, it only remains to check that

$$
y^{m_{r}} f_{r}-x f_{r-1} \in\left\langle f_{t_{k-1}}, f_{t_{k-1}+1}, \ldots, f_{r-1}\right\rangle .
$$

This can be done by using the same elimination procedure seen earlier in the proof of Lemma 3.4.13:

(1) Write $E_{1}=y^{m_{r}} f_{r}-x f_{r-1}$.

(2) Having obtained $E_{i}$, eliminate monomials divisible by $\alpha_{r-i}$ from $E_{i}$ by subtracting from $E_{i}$ a suitable multiple $p_{r-i} f_{r-i}$ of $f_{r-i}$ to obtain $E_{i+1}=E_{i}-p_{r-i} f_{r-i}$.

Observe that all the monomials occuring in each of the $E_{i}$ lie in the $\left(r+l_{r-1}\right)^{t h}$ diagonal. Since $r+l_{r-1}<k$, there are no monomials occuring in $E_{i}$ which are divisible by $\alpha_{j}$ for $j=0,1,2, \ldots, t_{k-1}-1$. Hence, $E_{r-t_{k-1}+1}$ already lies in the linear span of $\mathcal{P}\left(T_{1}\right)$ and satisfies

$$
E_{r-t_{k-1}+1}=y^{m_{r}} f_{r}-x f_{r-1} \quad \bmod \left\langle f_{t_{k-1}}, f_{t_{k-1}+1}, \ldots, f_{r-1}\right\rangle .
$$

So $E_{r-t_{k-1}+1}=0$ and $y^{m_{r}} f_{r}-x f_{r-1}$ is in $\left\langle f_{t_{k-1}}, f_{t_{k-1}+1}, \ldots, f_{r-1}\right\rangle$ as required.

Since $L_{T_{1}, T_{2}}$ is smooth and projective, any $\mathbb{C}^{*}$-action on $L_{T_{1}, T_{2}}$ induced by one of our $\mathbb{C}^{*}$-actions on $\mathcal{Z}_{n-1, n}\left(\mathbb{A}^{2}, 0\right)$ gives rise to a cellular decomposition of $L_{T_{1}, T_{2}}$ by the theorem of Bialynicki-Birula. The cells of $L_{T_{1}, T_{2}}$ are the intersections of $L_{T_{1}, T_{2}}$ with the cells of $M_{T_{1}, T_{2}}$.

Proposition 3.4.15. Let $X$ be the Hilbert function stratum $M_{T_{1}, T_{2}}$ of $\mathcal{Z}_{n-1, n}\left(\mathbb{A}^{2}, 0\right)$ or the related smooth projective variety $L_{T_{1}, T_{2}}$. Then,

(1) $X$ has a cellular decomposition; the map $A_{*}(X) \rightarrow H_{*}(X)$ is an isomorphism and $H_{*}(X)$ is free.

(2) The Betti number $b_{2 i}\left(M_{T_{1}, T_{2}}\right)$ is equal to the cardinality of the set of pairs of Young diagrams $\left(\Gamma_{1}, \Gamma_{2}\right)$ for which

$$
\begin{gathered}
\left(T\left(\Gamma_{1}\right), T\left(\Gamma_{2}\right)\right)=\left(T_{1}, T_{2}\right) \quad \text { and } \\
(n-1)-\left|\left\{(u, v) \in \Gamma_{1}: h_{u, v}\left(\Gamma_{1}\right) \in\{0,1\}\right\}\right|+\diamond^{+}\left(\Gamma_{1}, \Gamma_{2}\right)-\star \star^{+}\left(\Gamma_{1}, \Gamma_{2}\right)=i .
\end{gathered}
$$

(3) The Betti number $b_{2 i}\left(L_{T_{1}, T_{2}}\right)$ is equal to the cardinality of the set of pairs of Young diagrams $\left(\Gamma_{1}, \Gamma_{2}\right)$ for which

$$
\begin{gathered}
\left(T\left(\Gamma_{1}\right), T\left(\Gamma_{2}\right)\right)=\left(T_{1}, T_{2}\right) \quad \text { and } \\
\left|\left\{(u, v) \in \Gamma_{1}: h_{u, v}\left(\Gamma_{1}\right)=-1\right\}\right|+\diamond^{+}\left(\Gamma_{1}, \Gamma_{2}\right)-\star^{+}\left(\Gamma_{1}, \Gamma_{2}\right)=i .
\end{gathered}
$$


In particular, the Euler numbers are

$$
\chi\left(M_{T_{1}, T_{2}}\right)=\chi\left(L_{T_{1}, T_{2}}\right)=\left|\left\{\left(\Gamma_{1}, \Gamma_{2}\right): \Gamma_{1} \subset \Gamma_{2}, T\left(\Gamma_{i}\right)=T_{i}\right\}\right| .
$$

Proof. This follows from Theorem 3.0.2, Lemma 3.4.9.3, Lemma 3.4.9.4, Equation (3.4.1) and Equation (3.4.2).

\section{References}

[B1] A. Bialynicki-Birula, Some theorems on actions of algebraic groups, Ann. Math., 98 (1973), 480-497.

[B2] Some properties of the decompositions of algebraic varieties determined by actions of a torus, Bull. Acad. Pol. Sci. Sér. Sci. Math. Astron. Phys., 24(9) (1976), 667-674.

[Br] J. Briançon, Description de $\operatorname{Hilb}^{n} \mathbb{C}\{x, y\}$, Invent. Math., 41 (1977), 45-89.

[Ch1] J. Cheah, The cohomology of smooth nested Hilbert schemes of points, thesis, Chicago, 1994.

[Ch2] - On the cohomology of Hilbert schemes of points, J. Algebraic Geometry, 5 (1996), 479-511.

[Ch3] - The virtual Hodge polynomials of nested Hilbert schemes of points and related varieties, 1998, to appear in Math. Z.

[Elen-LeB] G. Elencwajg and P. Le Barz, Explicit computations in Hilb $\mathbb{P}^{2}$, Proc. Alg. Geom. Sundance, 1986 Springer, LNM 1311 (1988), 76-100.

[E-S] G. Ellingsrud and S.A. Strømme, On the homology of the Hilbert scheme of points in the plane, Invent. Math., 87 (1987), 343-352.

[Fo] J. Fogarty, Algebraic families on an algebraic surface, Am. J. Math., 90 (1968), 511-521.

[Fu] W. Fulton, Intersection theory, Ergebnisse der Mathematik und ihrer Grenzgebiete, Springer, 1984.

[Gö1] L. Göttsche, Betti numbers for the Hilbert function strata of the punctual Hilbert scheme in two variables, Manuscripta Math., 66 (1990), 253-259.

[Gö2] _ Hilbert schemes of zero-dimensional subschemes of length $n$ of a smooth projective variety, thesis - English translation, 1991, Bonn.

[Gr] A. Grothendieck, Techniques de construction et théorèmes d'existence en géométrie algébrique IV: Les schémas de Hilbert, Séminaire Bourbaki, IHP, Paris, 221 (1961).

[Iar] A. Iarrobino, Punctual Hilbert schemes, Mem. Amer. Math. Soc., 188 (1977).

[Iar-Ya1] A. Iarrobino and J. Yaméogo, Graded ideals in $k[x, y]$ and partitions I: Partitions of diagonal lengths and the hook code, preprint, 1994.

[Iar-Ya2] _ Graded ideals in $k[x, y]$ and partitions II: Ramification and a generalization of Schubert calculus, preprint, 1994.

[Kol] J. Kollár, Rational curves on algebraic varieties, preprint, 1994.

[Mum] D. Mumford, Lectures on curves on an algebraic surface, 1966. 
[Tik] A.S. Tikhomirov, On Hilbert schemes and flag varieties of points on algebraic surfaces, preprint, 1992.

Received October 7, 1994 and revised August 15, 1997. During this research, the author was supported by a Sloan Foundation doctoral dissertation fellowship.

UNIVERSITY OF FLORIDA

Gainesville, FL 32611

E-mail address: jlc@math.ufl.edu

Note: The Reference in the Proof of Proposition 2.6.4 TO Lemma 2.6.2 APPEARED IN THE PAPER VERSION AS "PROPOSITION 2.6.2". 